\title{
Mechanistic insights into the racemization of fused cyclopropyl isoxazolines.
}

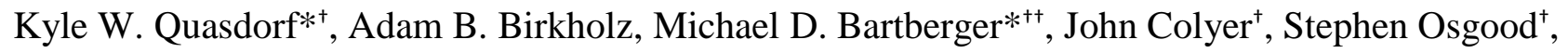 \\ Kevin Crossley ${ }^{\dagger}$, and Seb Caille ${ }^{\dagger}$ \\ ${ }^{\dagger}$ Departments of Chemical Process Research and Development and Therapeutic Discovery, Amgen \\ Inc., One Amgen Center Drive, Thousand Oaks, California 91320 \\ ${ }^{+\dagger} 1200$ Pharma LLC, 844 East Green Street, Suite 204, Pasadena, CA 91101 \\ $\underline{\text { Supporting Information - Table of Contents }}$
}

Materials and Methods .S1

Experimental Procedures............................................................................................................................S2

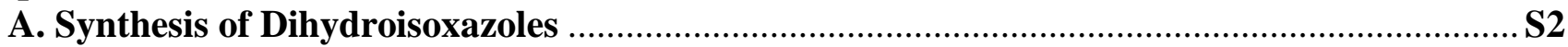

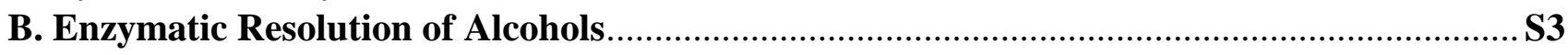

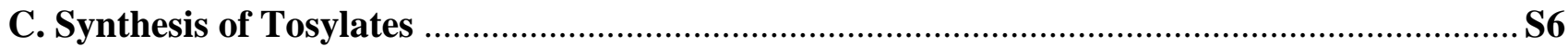

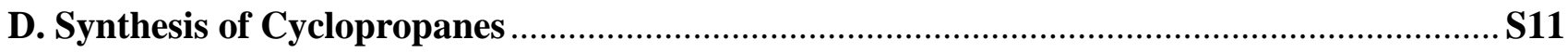

E. Racemization Studies of Cyclopropanes ....................................................................... S14

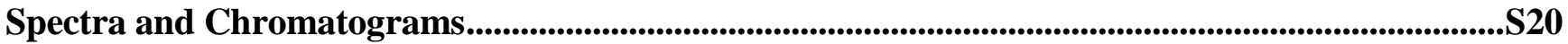

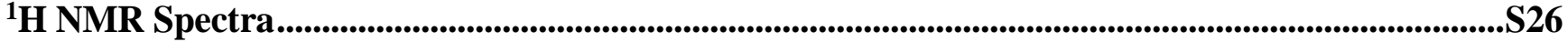

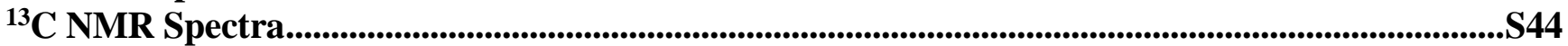

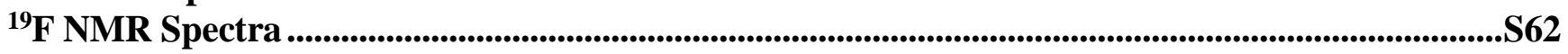

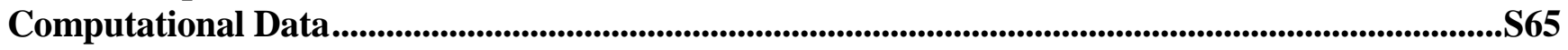

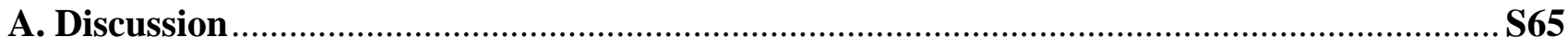

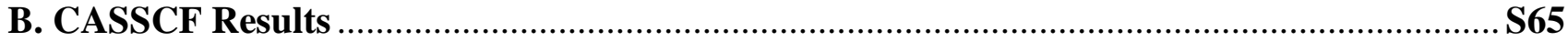

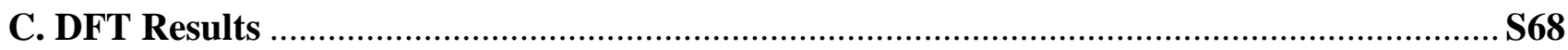

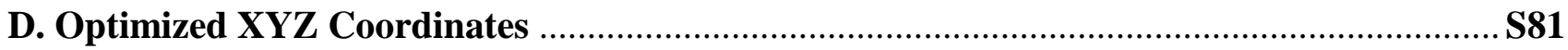

Materials and Methods. Unless stated otherwise, reactions were conducted under an atmosphere of nitrogen or argon using anhydrous solvents. All commercially obtained reagents were used as received. Reaction temperatures were controlled using an IKAmag temperature modulator, and unless stated otherwise, reactions were performed at room temperature (rt, approximately $21{ }^{\circ} \mathrm{C}$ ). Pre-packed RediSepR $_{f}$ silica columns were used for flash column chromatography along with a Teledyne CombiFlashR $_{f} 200 i$ instrument. ${ }^{1} \mathrm{H}$ NMR spectra were recorded on Bruker spectrometers (at $400 \mathrm{MHz}$ ) and are reported relative to residual protonated solvent signals $\left(\delta 7.27\right.$ for $\mathrm{CDCl}_{3}$ and $\delta 7.16$ for $\left.\mathrm{C}_{6} \mathrm{D}_{6}\right)$. Data for ${ }^{1} \mathrm{H}$ NMR spectra are reported as follows: chemical shift ( $\left.\delta \mathrm{ppm}\right)$, multiplicity, coupling constant (Hz) and integration. ${ }^{13} \mathrm{C}$ NMR spectra were recorded on Bruker Spectrometers (at $100 \mathrm{MHz}$ ) and are reported relative to and are reported relative to deuterated solvent signals $\left(\delta 77.0\right.$ for $\mathrm{CDCl}_{3}$ and $\delta 128.4$ for $\mathrm{C}_{6} \mathrm{D}_{6}$ ). Data for ${ }^{13} \mathrm{C}$ NMR spectra are reported in terms of chemical shift. ${ }^{19} \mathrm{~F}$ NMR spectra were recorded on Bruker Spectrometers (at $376 \mathrm{MHz}$ ) and are reported relative to internal instrument 
default chemical shift reference of $\mathrm{CFCl}_{3}$ liquid at $0.00 \mathrm{ppm}$. Data for ${ }^{19} \mathrm{~F}$ NMR spectra are reported in terms of chemical shift. Optical rotations were measured with a Rudolph Autopol V polarimeter. Highresolution mass spectra were obtained with a Thermo Q-Exactive HF-X spectrometer.

\section{Experimental Procedures.}

\section{A. Synthesis Of Dihydroisoxazoles}

Dihydroisoxazole SI-2 was prepared from following procedure. Compounds $r a c-S I-3^{\mathbf{1}}, r a c_{-}-\mathbf{S I}^{-4}{ }^{\mathbf{1}}, \mathrm{rac}^{-}$ SI-5 ${ }^{2}$, and rac-SI-6 $^{\mathbf{3}}$ were known from the literature.

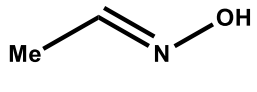

SI-1

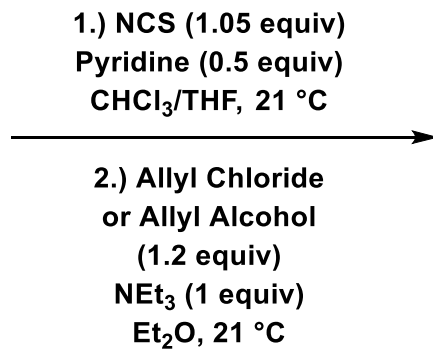

( $57 \%$ yield, 2 steps)

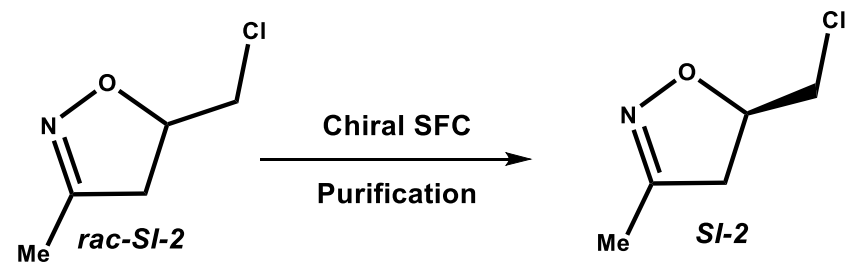

Methyl dihydroisoxazole SI-2. A round-bottom flask was charged with acetaldehyde oxime (SI-1) (15.0 $\mathrm{g}, 254 \mathrm{mmol}, 1$ equiv), followed by the addition of $\mathrm{CHCl}_{3}(180 \mathrm{~mL})$ and $\mathrm{THF}(180 \mathrm{~mL})$. To the resulting solution was added NCS (36.3 g, $267 \mathrm{mmol}, 1.05$ equiv) in two separate portions of $18.15 \mathrm{~g}$ over $10 \mathrm{~min}$. Then pyridine (10.0 g (10.3 mL), $127 \mathrm{mmol}, 0.5$ equiv) was added at such a rate to keep the internal reaction temperature below $35^{\circ} \mathrm{C}$. The mixture was then stirred at room temperature for $5 \mathrm{~h}$. After which time the mixture was filtered through a pad of silica gel washed with $\mathrm{CHCl}_{3}(100 \mathrm{~mL})$ and the filtrate concentrated under reduced pressure to obtain a crude orange oil which was used directly in the next step.

\footnotetext{
${ }^{1}$ Castello, S.; Kuck, D.; Viviano, M.; Yoo, J.; Lopez-Vallejo, F.; Conti, P.; Tamborini, L.; Pinto, A.; MedinaFranco, J.; Sbardella, G. J. Med. Chem. 2011, 54, 7663-7677.

2 Li, W.; Jia, P.; Han, B.; Li, D.; Yu, W. Tetrahedron. 2013, 69, 3274-3280.

${ }^{3}$ Sugak, H.; Adachi, Y.; Fujimoto, K.; Furihata, Y.; Tsuchida, T.; Kakehi, A.; Baba, T. J. Org. Chem. 2009, 74, 1099-1113.
} 
To a 3-neck round-bottom flask containing the crude material from the previous step was added $\mathrm{Et}_{2} \mathrm{O}$ (540 mL) and $\mathrm{CHCl}_{3}(40 \mathrm{~mL})$. The flask was then fitted with an overhead stirrer followed by the addition of allyl chloride (23.3 g (24.8 mL), 305 mmol, 1.2 equiv). The NEt 3 (25.7 g (35.4 mL), 254 mmol, 1 equiv) was then added dropwise at such a rate to keep the internal reaction temperature below $30{ }^{\circ} \mathrm{C}$. After the addition was complete the mixture was stirred for $4 \mathrm{~h}$. After which time the mixture was filtered through a pad of Celite washed with $\mathrm{Et}_{2} \mathrm{O}(150 \mathrm{~mL})$ and concentrated under reduced pressure. The crude residue was transferred to a separatory funnel with $\mathrm{Et}_{2} \mathrm{O}(200 \mathrm{~mL})$ and water $(50 \mathrm{~mL})$. The resulting biphasic mixture was extracted with $\mathrm{Et}_{2} \mathrm{O}(2 \times 50 \mathrm{~mL})$ and the organic layers were combined, dried over $\mathrm{MgSO}_{4}$, filtered through a silica pad (washed with $100 \mathrm{~mL}$ of $\mathrm{Et}_{2} \mathrm{O}$ ), and evaporated under reduced pressure to yield rac-methyl dihydroisoxazole rac-SI-2 (19.4 g, 57\% yield, over 2 steps) as a yellow oil. Methyl dihydroisoxazole rac-SI-2 was further purified by preparative SFC using an AD-H column with $7 \% \mathrm{MeOH}$ in $\mathrm{CO}_{2}$ as eluent with a flow rate of $100 \mathrm{~mL} / \mathrm{min}$ to obtain product $\mathbf{S I - 2}$ with $>98 \% e e .{ }^{4} c=$ $0.053\left(\mathrm{CHCl}_{3}\right),[\alpha]_{\mathrm{D}}{ }^{22.2}-16.92 ;{ }^{1} \mathrm{H} \mathrm{NMR}\left(400 \mathrm{MHz}, \mathrm{CDCl}_{3}\right): \delta 4.81-4.74(\mathrm{~m}, 1 \mathrm{H}), 3.62-3.58(\mathrm{dd}, J=$ $12.0,4.0,1 \mathrm{H}), 3.50-3.46(\mathrm{dd}, J=12.0,8.0,1 \mathrm{H}), 3.12-3.05(\mathrm{ddd}, J=16.0,12.0,4.0,1 \mathrm{H}), 2.91-2.85$ $(\mathrm{ddd}, J=16.0,8.0,4.0,1 \mathrm{H}), 1.98(\mathrm{~s}, 3 \mathrm{H}) ;{ }^{13} \mathrm{C} \mathrm{NMR}\left(100 \mathrm{MHz}, \mathrm{CDCl}_{3}\right): \delta 154.9,78.7,44.9,42.1,12.8$.

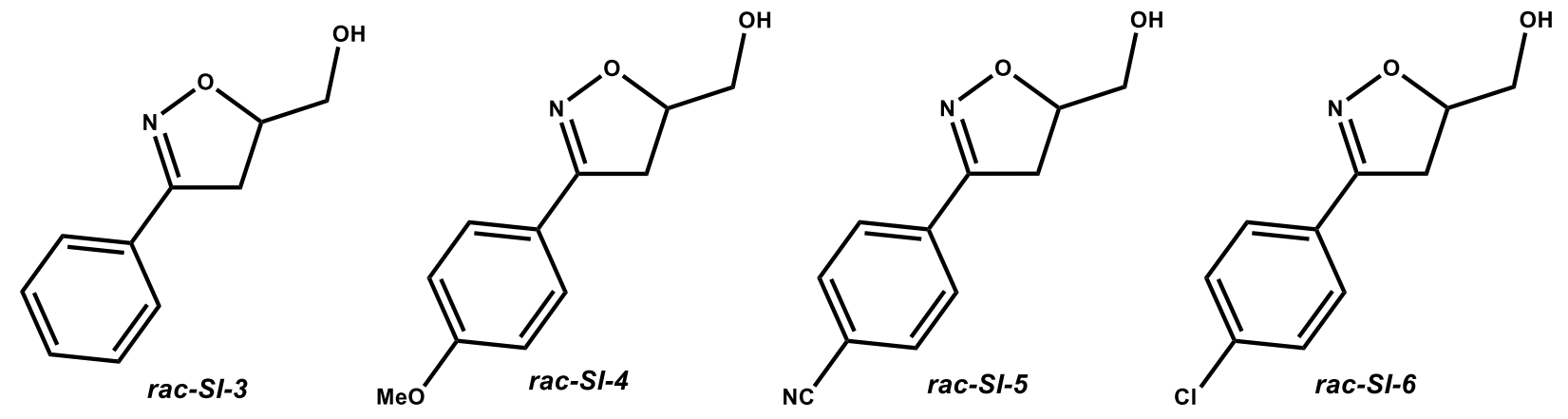

\section{B. Enzymatic Resolution Of Alcohols}

\footnotetext{
${ }^{4}$ Absolute stereochemistry is assigned by analogy base on the optical rotation of the corresponding alcohol compound. Curran. D. P.; Kim, B.; Daugherty, J.; Heffner, T. A. Tetrahedron Lett. 1988, 29, 35553558.
} 

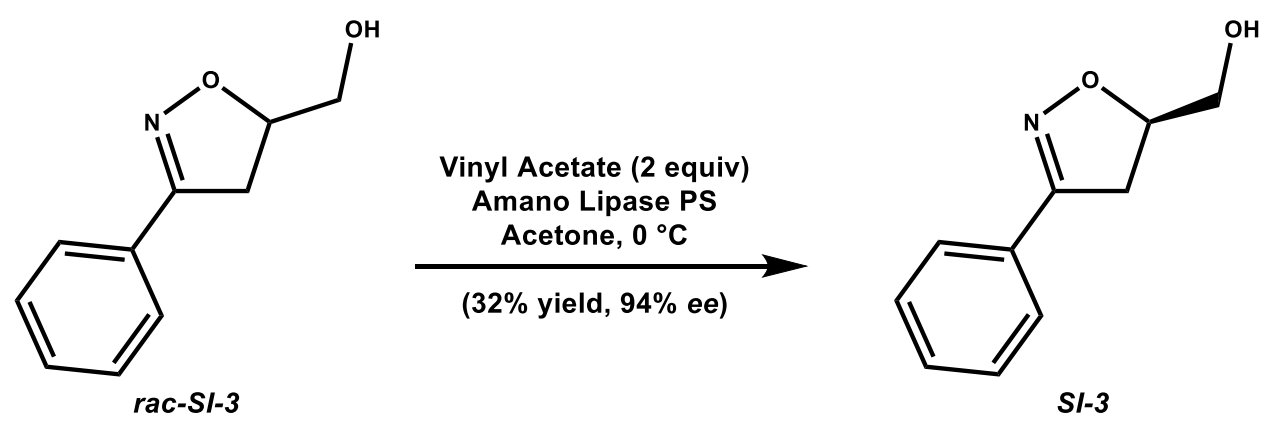

Alcohol SI-3. A round-bottom flask was charged with alcohol rac-SI-3 (3.16 g, 17.83 mmol) and Amano Lipase PS-IM (immobilized on diatomaceous earth) $(1.05 \mathrm{~g})$. Acetone $(265 \mathrm{~mL})$ was added and the mixture was cooled to $0{ }^{\circ} \mathrm{C}$, followed by the addition of vinyl acetate $(3.07 \mathrm{~g}, 35.66 \mathrm{mmol}, 2$ equiv) and left to stir for $18 \mathrm{~h}$. The reaction was monitored by LCMS and was stopped at $50 \%$ conversion, after which time the mixture was filtered and evaporated under reduced pressure. The crude residue was purified by automated flash chromatography (1:1 heptanes: EtOAc) to yield alcohol SI-3 (1.00 g, 32\% yield, $94 \% e e)$ as a white solid. $c=0.09\left(\mathrm{CHCl}_{3}\right),[\alpha]_{\mathrm{D}}{ }^{21.3}-38.53 .^{5}$
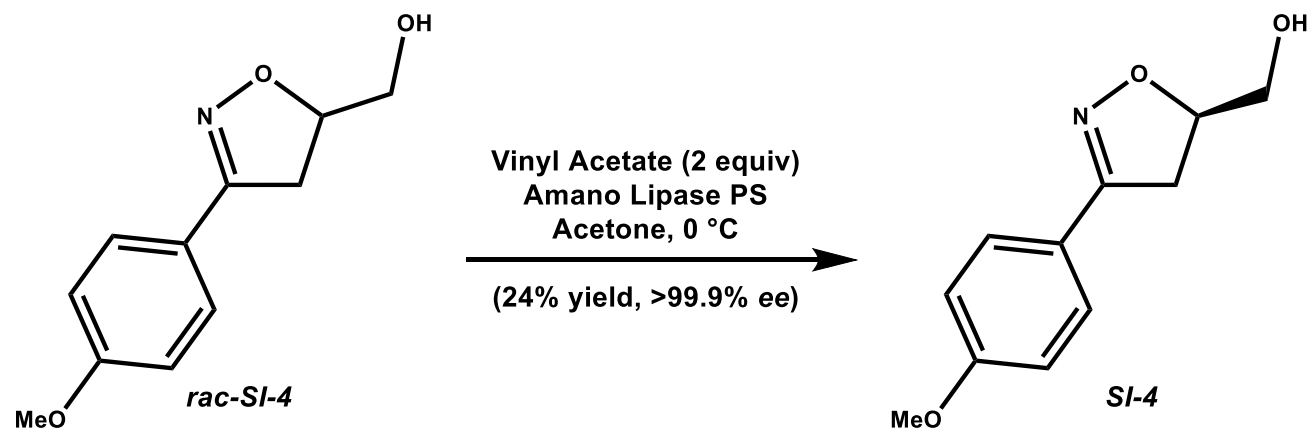

Alcohol SI-4. A round-bottom flask was charged with alcohol rac-SI-4 (5.0 g, 24.13 mmol) and Amano Lipase PS-IM (immobilized on diatomaceous earth) $(1.67 \mathrm{~g})$. Acetone $(420 \mathrm{~mL})$ was added and the mixture was cooled to $0{ }^{\circ} \mathrm{C}$, followed by the addition of vinyl acetate $(4.15 \mathrm{~g}, 48.26 \mathrm{mmol}, 2$ equiv) and left to stir for 2 hours. Progress was monitored by LCMS with additional 10\% charges of amano lipase PS-IM after 2 and 3 hours. The reaction was stopped at $60 \%$ conversion at 4 hours, after which time the mixture was filtered and evaporated under reduced pressure. The crude residue was purified by

\footnotetext{
${ }^{5}$ For optical rotation data see; (a) Serizawa, M.; Ukaji, Y.; Inomata, K. Tetrahedron: Asymmetry. 2006, 17, 3075-3083. (b) Sugak, H.; Adachi, Y.; Fujimoto, K.; Furihata, Y.; Tsuchida, T.; Kakehi, A.; Baba, T. J. Org. Chem. 2009, 74, 1099-1113.
} 
automated flash chromatography (1:1 heptanes: EtOAc) to yield alcohol SI-4 (1.2 g, 24\% yield, >99.9\% $e e)$ as a white solid. $c=0.006\left(\mathrm{CHCl}_{3}\right),[\alpha]_{\mathrm{D}}{ }^{22.6}-2.27 .^{5}$
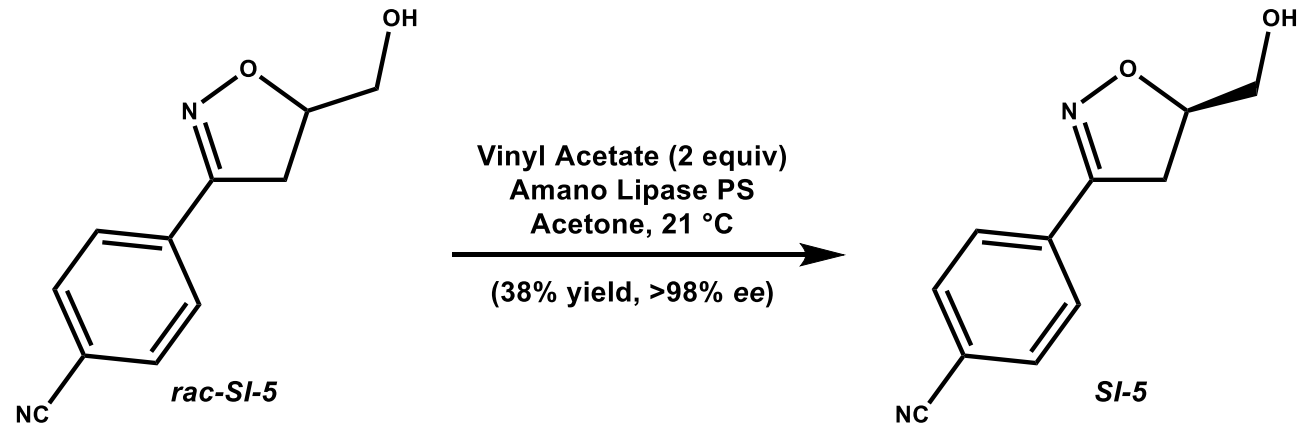

Alcohol SI-5. A round-bottom flask was charged with alcohol rac-SI-5 (9.0 g, 44.5 mmol) and Amano Lipase PS-IM (immobilized on diatomaceous earth) (3 g). Acetone $(750 \mathrm{~mL})$ was added and the mixture was left to stir at room temperature, followed by the addition of vinyl acetate $(7.66 \mathrm{~g}, 89 \mathrm{mmol}, 2$ equiv) and left to stir overnight. Progress was monitored by LCMS with additional 100\% w/w charges of amano lipase PS-IM after 20 and 42 hours. The reaction was stopped at 57\% conversion at 48 hours, after which time the mixture was filtered and evaporated under reduced pressure. The crude residue was purified by automated flash chromatography (1:1 heptanes: EtOAc) to yield alcohol SI-5 (3.4 g, 38\% yield, >98\% $e e)$ as a white solid. $c=0.11\left(\mathrm{CHCl}_{3}\right),[\alpha]_{\mathrm{D}}{ }^{21.4}-45.33 .^{6}$
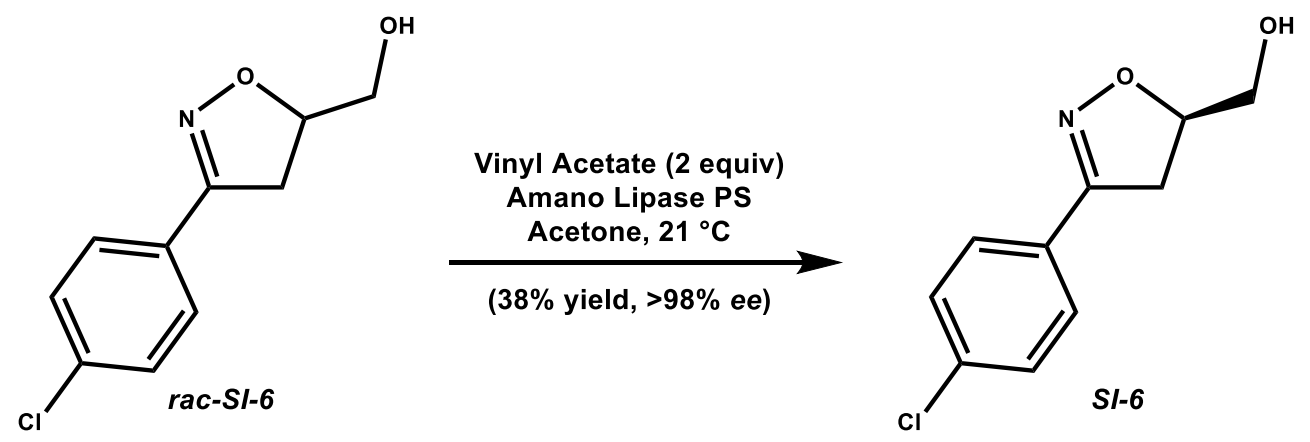

Alcohol SI-6. A round-bottom flask was charged with alcohol rac-SI-6 (5.55 g, $26.2 \mathrm{mmol}$ ) and Amano Lipase PS-IM (immobilized on diatomaceous earth) $(1.85 \mathrm{~g})$. Acetone (462 mL) was added and the mixture was left to stir at room temperature, followed by the addition of vinyl acetate $(4.52 \mathrm{~g}, 52.5 \mathrm{mmol}$, 2 equiv) and left to stir overnight. Progress was monitored by LCMS with additional 100\% w/w charges of amano lipase PS-IM after 24 hours. The reaction was stopped at 50\% conversion at 48 hours, after

${ }^{6}$ For optical rotation data see; Serizawa, M.; Ukaji, Y.; Inomata, K. Tetrahedron: Asymmetry. 2006, 17, 3075-3083. 
which time the mixture was filtered and evaporated under reduced pressure. The crude residue was purified by automated flash chromatography (1:1 heptanes: EtOAc) to yield alcohol SI-6 (1.95 g, 35\% yield, $>98 \% e e)$ as a white solid. $c=0.065\left(\mathrm{CHCl}_{3}\right),[\alpha]_{\mathrm{D}}{ }^{20.7}-30.40 .^{7}$

\section{Synthesis Of Tosylates}

Tosylates SI-7, SI-8, SI-9, and SI-10 we synthesized following the general procedures below.

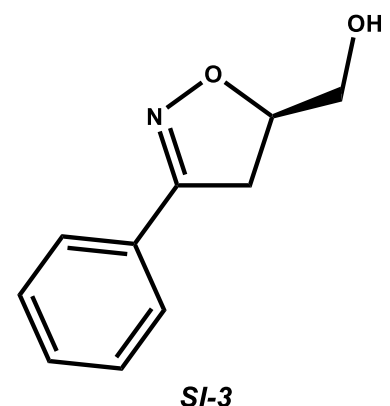

SI-3

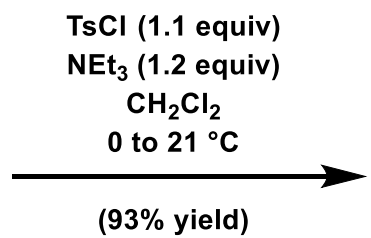

$(93 \%$ yield $)$

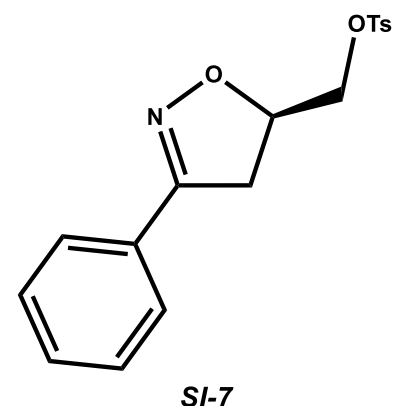

SI-7

Tosylate SI-7. A round-bottom flask was charged with phenyl dihydroisoxazole SI-3 (1.38 g, $7.80 \mathrm{mmol})$ followed by the addition of $\mathrm{CH}_{2} \mathrm{Cl}_{2}(40 \mathrm{~mL})$ and then $\mathrm{NEt}_{3}(0.95 \mathrm{~g}(1.3 \mathrm{~mL}), 9.40 \mathrm{mmol}, 1.2 \mathrm{equiv})$. The solution was then cooled to $0{ }^{\circ} \mathrm{C}$ and tosyl chloride (1.64 g, $8.60 \mathrm{mmol}, 1.1$ equiv.) was added and the solution was allowed to stir and slowly warm to room temperature over $16 \mathrm{~h}$. After which time the reaction mixture was transferred to a separatory funnel with EtOAc $(50 \mathrm{~mL})$, water $(10 \mathrm{~mL})$, and saturated aqueous $\mathrm{NH}_{4} \mathrm{Cl}$ solution $(25 \mathrm{~mL})$. The resulting biphasic mixture was extracted with EtOAc (2 x $50 \mathrm{~mL}$ ) and the organic layers were combined, dried over $\mathrm{MgSO}_{4}$, and evaporated under reduced pressure. The crude residue was purified by automated flash chromatography (80 g column, 100\% heptanes to $100 \%$ EtOAc gradient over $30 \mathrm{~min}$ ) to yield tosylate SI-7 (2.39 g, 93\% yield) as a white solid. ${ }^{1} \mathrm{H}$ NMR (400 MHz, $\mathrm{CDCl}_{3}$ ): $\delta 7.80$ (d, $\left.J=8.0,2 \mathrm{H}\right), 7.64-7.62(\mathrm{~m}, 2 \mathrm{H}), 7.44-7.41$ (m, 3H), 7.35 $(\mathrm{d}, J=8.0,2 \mathrm{H}), 4.98-4.91(\mathrm{~m}, 1 \mathrm{H}), 4.21-4.17(\mathrm{dd}, J=8.0,4.0,1 \mathrm{H}), 4.12-4.08(\mathrm{dd}, J=8.0,4.0,1 \mathrm{H})$, $3.49-3.42(\mathrm{dd}, J=16.0,12.0,1 \mathrm{H}), 3.29-3.23(\mathrm{dd}, J=20.0,4.0,1 \mathrm{H}), 2.45(\mathrm{~s}, 3 \mathrm{H}) .{ }^{13} \mathrm{C} \mathrm{NMR}(100 \mathrm{MHz}$, $\left.\mathrm{CDCl}_{3}\right): \delta 156.2,145.2,132.5,130.4,130.0,128.83,128.75,128.0,126.8,77.4,69.2,37.3,21.6$; HRMSESI $(\mathrm{m} / \mathrm{z})[\mathrm{M}+\mathrm{H}]^{+}$calculated for $\mathrm{C}_{17} \mathrm{H}_{18} \mathrm{NO}_{4} \mathrm{~S}, 332.09511$; found, 332.09564 .

${ }^{7}$ For optical rotation data see; Sugak, H.; Adachi, Y.; Fujimoto, K.; Furihata, Y.; Tsuchida, T.; Kakehi, A.; Baba, T. J. Org. Chem. 2009, 74, 1099-1113. 


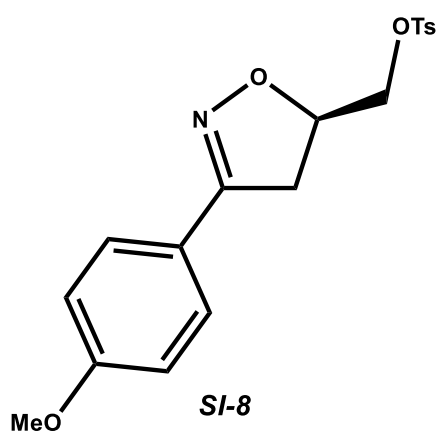

Tosylate SI-8. Quantitative yield, white solid. ${ }^{1} \mathrm{H}$ NMR (400 MHz, $\left.\mathrm{CDCl}_{3}\right): \delta 7.80(\mathrm{~d}, J=8.0,2 \mathrm{H}), 7.56$ (d, $J=8.0,2 \mathrm{H}), 7.35(\mathrm{~d}, J=8.0,2 \mathrm{H}), 6.92(\mathrm{~d}, J=8.0,2 \mathrm{H}), 4.94-4.87(\mathrm{~m}, 1 \mathrm{H}), 4.19-4.15(\mathrm{dd}, J=8.0$, $4.0,1 \mathrm{H}), 4.10-4.06(\mathrm{dd}, J=8.0,4.0,1 \mathrm{H}), 3.85$ (s, 3H), 3.46-3.39 (dd, $J=16.0,12.0,1 \mathrm{H}), 3.25-3.19$ (dd, $J=16.0,8.0,1 \mathrm{H}), 2.45$ (s, 3H). ${ }^{13} \mathrm{C}$ NMR $\left(100 \mathrm{MHz}, \mathrm{CDCl}_{3}\right): \delta 161.3,155.8,145.2,132.5,129.9$, 128.3, 128.0, 121.4, 114.12, 77.1, 69.2, 55.4, 37.6, 21.6; HRMS-ESI $(\mathrm{m} / \mathrm{z})[\mathrm{M}+\mathrm{H}]^{+}$calculated for $\mathrm{C}_{18} \mathrm{H}_{20} \mathrm{O}_{5} \mathrm{NS}, 362.10567$; found, 362.10623 .

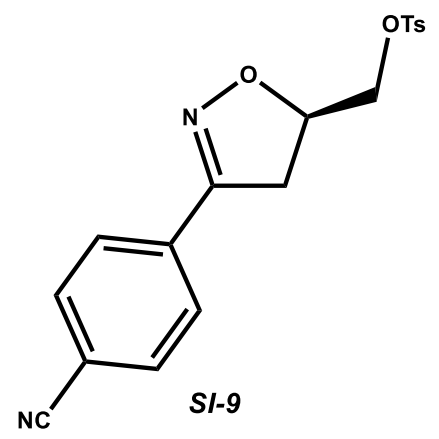

Tosylate SI-9. 46\% yield, white solid. ${ }^{1} \mathrm{H}$ NMR (400 MHz, $\left.\mathrm{CDCl}_{3}\right): \delta 7.71(\mathrm{~d}, J=8.0,2 \mathrm{H}), 7.68-7.62$ (m, 4H), $7.28(\mathrm{~d}, J=8.0,2 \mathrm{H}), 4.97-4.90(\mathrm{~m}, 1 \mathrm{H}), 4.16-4.12(\mathrm{dd}, J=12.0,4.0,1 \mathrm{H}), 4.09-4.05(\mathrm{dd}, J=$ $12.0,8.0,1 \mathrm{H}), 3.41-3.34(\mathrm{dd}, J=16.0,12.0,1 \mathrm{H}), 3.25-3.19(\mathrm{dd}, J=16.0,8.0,1 \mathrm{H}), 2.38(\mathrm{~s}, 3 \mathrm{H}) .{ }^{13} \mathrm{C}$ NMR (100 MHz, $\left.\mathrm{CDCl}_{3}\right): \delta 155.0,145.3,133.1,132.5,132.4,130.0,128.0,127.2,118.2,113.8,78.4$, 68.9, 36.5, 21.7; HRMS-ESI (m/z) [M + H] calculated for $\mathrm{C}_{18} \mathrm{H}_{17} \mathrm{O}_{4} \mathrm{~N}_{2} \mathrm{~S}, 357.09035$; found, 357.09080.

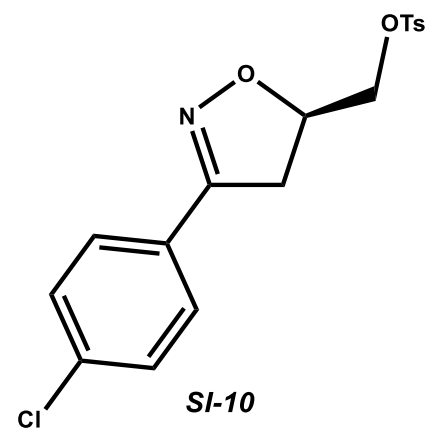


Tosylate SI-10. 93\% yield, white solid. ${ }^{1} \mathrm{H}$ NMR (400 $\left.\mathrm{MHz}, \mathrm{CDCl}_{3}\right): \delta 7.79(\mathrm{~d}, J=8.0,2 \mathrm{H}), 7.56(\mathrm{~d}, J$ $=8.0,2 \mathrm{H}), 7.39-7.34(\mathrm{~m}, 4 \mathrm{H}), 4.98-4.92(\mathrm{~m}, 1 \mathrm{H}), 4.21-4.17(\mathrm{dd}, J=12.0,4.0,1 \mathrm{H}), 4.13-4.09$ (dd, $J$ $=12.0,4.0,1 \mathrm{H}), 3.46-3.39(\mathrm{dd}, J=16.0,12.0,1 \mathrm{H}), 3.26-3.20(\mathrm{dd}, J=16.0,4.0,1 \mathrm{H}), 2.45(\mathrm{~s}, 3 \mathrm{H}) .{ }^{13} \mathrm{C}$ NMR (100 MHz, $\left.\mathrm{CDCl}_{3}\right): \delta 155.3,145.2,136.4,132.4,130.0,129.0,127.97,127.96,127.3,77.7,69.1$, 37.1, 21.6; HRMS-ESI (m/z) [M + Na $]^{+}$calculated for $\mathrm{C}_{17} \mathrm{H}_{16} \mathrm{O}_{4} \mathrm{NSClNa}, 388.03808$; found, 388.03827.
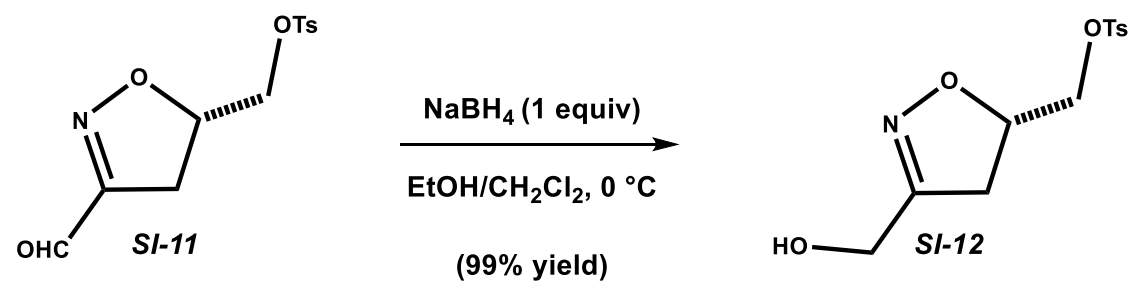

Alcohol SI-12. A round-bottom flask was charged with aldehyde SI-11 (5.00 g, 17.7 mmol, 1 equiv), followed by the addition of ethanol $(75 \mathrm{~mL})$ and $\mathrm{CH}_{2} \mathrm{Cl}_{2}(75 \mathrm{~mL})$. The resulting solution was then cooled to $0{ }^{\circ} \mathrm{C}$ and sodium borohydride (734 mg, $19.4 \mathrm{mmol}, 1.1$ equiv) was added in two $367 \mathrm{mg}$ portions. The reaction temperature was maintained at $0{ }^{\circ} \mathrm{C}$ while the mixture was stirred for $30 \mathrm{~min}$. The reaction was then quenched by the slow addition of saturated aqueous $\mathrm{NH}_{4} \mathrm{Cl}$ solution $(50 \mathrm{~mL})$. The mixture was allowed to warm to room temperature and was then transferred to a separatory funnel with $\mathrm{Et}_{2} \mathrm{O}(50 \mathrm{~mL})$ and water $(40 \mathrm{~mL})$. The resulting biphasic mixture was extracted with $\mathrm{Et}_{2} \mathrm{O}(3 \times 50 \mathrm{~mL})$ and the organic layers were combined, dried over $\mathrm{MgSO}_{4}$, filtered through a silica pad (washed with $100 \mathrm{~mL} \mathrm{of} \mathrm{Et}_{2} \mathrm{O}$ ), and evaporated under reduced pressure to yield alcohol SI-12 (5.02 g, 99\% yield) as a white solid. ${ }^{1} \mathrm{H}$ NMR (400 MHz, $\left.\mathrm{CDCl}_{3}\right): \delta 7.78(\mathrm{~d}, J=8.0,2 \mathrm{H}), 7.36(\mathrm{~d}, J=8.0,2 \mathrm{H}), 4.84-4.77(\mathrm{~m}, 1 \mathrm{H}), 4.42-4.34$ (m, 2H), 4.11-4.07 (dd, $J=8.0,4.0,1 \mathrm{H}), 4.06-4.03(\mathrm{dd}, J=8.0,4.0,1 \mathrm{H}), 3.20-3.13(\mathrm{dd}, J=20.0,12.0$, 1H), 2.99-2.93 (dd, $J=20.0,8.0,1 \mathrm{H}), 2.45(\mathrm{~s}, 3 \mathrm{H}) ;{ }^{13} \mathrm{C} \mathrm{NMR}\left(100 \mathrm{MHz}, \mathrm{CDCl}_{3}\right): \delta 158.3,145.3,132.3$, 130.0, 127.9, 77.1, 69.5, 57.7, 37.1, 21.6; HRMS-ESI (m/z) $[\mathrm{M}+\mathrm{Na}]^{+}$calculated for $\mathrm{C}_{12} \mathrm{H}_{15} \mathrm{O}_{5} \mathrm{NSNa}$, 308.05631; found, 308.05685.
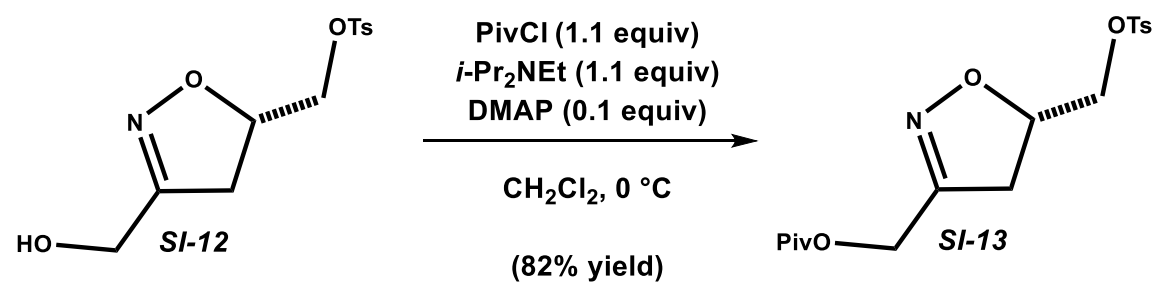
Pivalate SI-13. A round-bottom flask was charged with alcohol SI-12 (2.00 g, $7.01 \mathrm{mmol}, 1$ equiv), followed by the addition of $\mathrm{CH}_{2} \mathrm{Cl}_{2}(15 \mathrm{~mL}), i-\operatorname{Pr}_{2} \mathrm{NEt}(1.00 \mathrm{~g},(1.35 \mathrm{~mL}), 7.75 \mathrm{mmol}, 1.1$ equiv), and DMAP (0.084 g, $0.069 \mathrm{mmol}, 0.1$ equiv). The resulting solution was then cooled to $0{ }^{\circ} \mathrm{C}$ in an ice bath and pivaloyl chloride $(0.930 \mathrm{~g},(0.950 \mathrm{~mL}), 7.71 \mathrm{mmol}, 1.1$ equiv) was added dropwise. After the addition was complete the reaction was removed from the ice bath and allowed to stir a room temperature for $30 \mathrm{~min}$. After which time the reaction mixture was transferred to a separatory funnel with EtOAc (50 $\mathrm{mL})$, water $(10 \mathrm{~mL})$, and saturated aqueous $\mathrm{NH}_{4} \mathrm{Cl}$ solution $(25 \mathrm{~mL})$. The resulting biphasic mixture was extracted with EtOAc $(2 \times 50 \mathrm{~mL})$ and the organic layers were combined, dried over $\mathrm{MgSO}_{4}$, and evaporated under reduced pressure. The crude residue was purified by automated flash chromatography (80 g column, $100 \%$ heptanes to $60 \%$ heptanes / 40\% EtOAc gradient over $30 \mathrm{~min}$ ) to yield pivalate SI$13\left(2.13 \mathrm{~g}, 82 \%\right.$ yield) as a white solid. ${ }^{1} \mathrm{H}$ NMR (400 MHz, $\left.\mathrm{C}_{6} \mathrm{D}_{6}\right): \delta 7.70(\mathrm{~d}, J=8.0,2 \mathrm{H}), 6.69(\mathrm{~d}, J=$ $8.0,2 \mathrm{H}), 4.54-4.47(\mathrm{~m}, 2 \mathrm{H}), 4.20-4.14(\mathrm{~m}, 1 \mathrm{H}), 3.75-3.68(\mathrm{~m}, 2 \mathrm{H}), 2.40-2.34(\mathrm{dd}, J=20.0,8.0,1 \mathrm{H})$, 2.30-2.23 (dd, $J=16.0,12.0,1 \mathrm{H}), 1.82(\mathrm{~s}, 3 \mathrm{H}), 1.12(\mathrm{~s}, 9 \mathrm{H}) ;{ }^{13} \mathrm{C} \mathrm{NMR}\left(100 \mathrm{MHz}, \mathrm{C}_{6} \mathrm{D}_{6}\right): \delta 177.5$, 154.4, 144.9, 134.0, 130.3, 128.5, 77.9, 69.8, 58.7, 39.2, 37.3, 27.5, 21.5; HRMS-ESI (m/z) $[\mathrm{M}+\mathrm{Na}]^{+}$ calculated for $\mathrm{C}_{17} \mathrm{H}_{23} \mathrm{O}_{6} \mathrm{NSNa}, 392.11383$; found, 392.11459 .
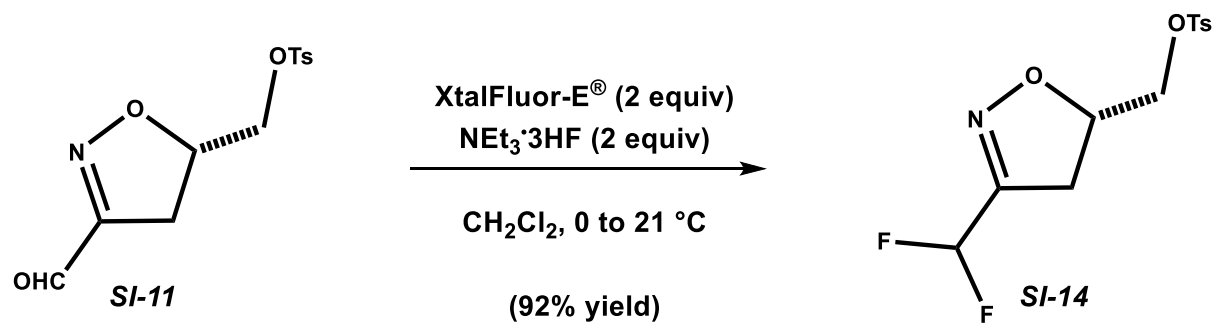

Tosylate SI-14. A round-bottom flask was charged with aldehyde SI-11 (5.00 g, 17.7 mmol, 1 equiv), followed by the addition of $\mathrm{CH}_{2} \mathrm{Cl}_{2}(50 \mathrm{~mL})$. The resulting solution was then cooled to $0{ }^{\circ} \mathrm{C}$ and XtalFluor-E $^{\circledR}$ (8.35 g, $36.5 \mathrm{mmol}, 2$ equiv) was added in one portion as a solid. The $\mathrm{NEt}_{3} \cdot 3 \mathrm{HF}(5.81 \mathrm{~g}$ $(5.87 \mathrm{~mL}), 36.5 \mathrm{mmol}, 2$ equiv) was then added dropwise and after the addition was complete the reaction was allowed to warm to room temperature and stirred for $3 \mathrm{~h}$. After which time a separate flask was charged with $\mathrm{CH}_{2} \mathrm{Cl}_{2}(30 \mathrm{~mL})$ and $5 \mathrm{M} \mathrm{NaOH}(30 \mathrm{~mL})$ then cooled to $0{ }^{\circ} \mathrm{C}$. The crude reaction mixture was then transferred to an addition funnel and added dropwise to the $\mathrm{CH}_{2} \mathrm{Cl}_{2} / 5 \mathrm{M} \mathrm{NaOH}$ mixture at such a rate to keep the internal temperature below $15^{\circ} \mathrm{C}$. After the addition was complete the mixture was transferred to a separatory funnel with MTBE $(100 \mathrm{~mL})$ and water $(50 \mathrm{~mL})$. The resulting biphasic mixture was extracted with $\operatorname{MTBE}(2 \times 50 \mathrm{~mL})$ and the organic layers were combined, dried over $\mathrm{MgSO}_{4}$, filtered through a silica pad (washed with $100 \mathrm{~mL}$ of MTBE), and evaporated under reduced pressure. The crude residue was purified by automated flash chromatography ( $220 \mathrm{~g}$ column, $100 \%$ heptanes to 
$100 \%$ EtOAc gradient over $30 \mathrm{~min})$ to yield tosylate SI-14 (4.98 g, 92\% yield) as a yellow oil. ${ }^{1} \mathrm{H}$ NMR (400 MHz, $\left.\mathrm{CDCl}_{3}\right): \delta 7.79(\mathrm{~d}, J=8.0,2 \mathrm{H}), 7.37(\mathrm{~d}, J=8.0,2 \mathrm{H}), 6.43\left(\mathrm{t}, J_{H-F}=56.0,1 \mathrm{H}\right), 4.98-4.92$ (m, 1H), 4.16-4.08 (m, 2H), 3.27-3.18 (m, 1H), 3.05-2.97 (m, 1H), 2.46 (s, 3H); ${ }^{13} \mathrm{C}$ NMR (100 MHz, $\left.\mathrm{CDCl}_{3}\right): \delta 152.7\left(\mathrm{t}, J_{C}{ }_{F}=31.0\right), 145.4,132.3,130.0,128.0,109.4\left(\mathrm{t}, J_{C}{ }_{F}=235.0\right), 78.7,68.7,33.0$, 21.7; ${ }^{19} \mathrm{~F}$ NMR $\left({ }^{1} \mathrm{H}\right.$ decoupled) $\left(377 \mathrm{MHz}, \mathrm{CDCl}_{3}\right): \delta-118.8\left(\mathrm{~d}, J_{F}{ }_{F}=324.2,1 \mathrm{~F}\right),-119.8\left(\mathrm{~d}, J_{F^{-}} F=\right.$ 328.0, 1F); HRMS-ESI (m/z) [M + H] $]^{+}$calculated for $\mathrm{C}_{12} \mathrm{H}_{14} \mathrm{O}_{4} \mathrm{NF}_{2} \mathrm{~S}, 306.06061$; found, 306.06119 .

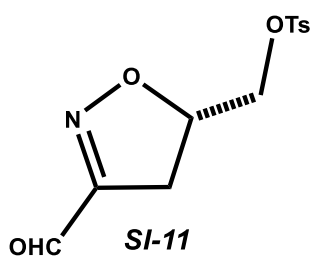

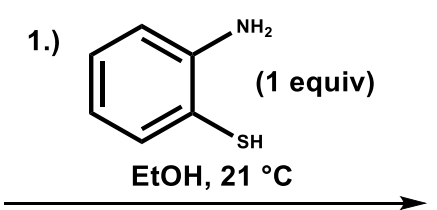

2.) Chloranil ( 1.05 equiv) $\mathrm{CH}_{2} \mathrm{Cl}_{2}, 21^{\circ} \mathrm{C}$

(84\% yield, 2 steps)

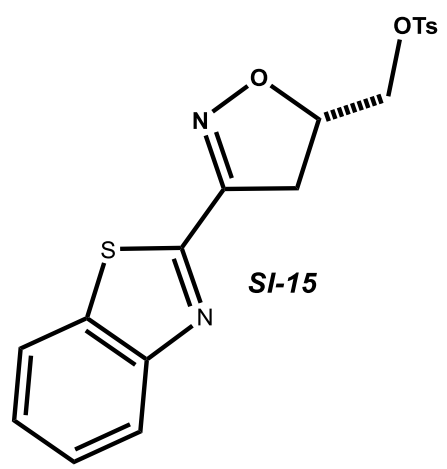

Tosylate SI-15. A round-bottom flask was charged with aldehyde SI-11 (5.00 g, 17.7 mmol, 1 equiv), followed by the addition of ethanol $(50 \mathrm{~mL})$. To resulting suspension was added 2-aminobenzenethiol ( $2.21 \mathrm{~g}, 17.7 \mathrm{mmol}, 1.0$ equiv). The reaction was allowed stir at $21^{\circ} \mathrm{C}$ for $16 \mathrm{~h}$. The resulting suspension was then concentrated under reduced pressure to remove the residual solvent. The crude residue was then concentrated under reduced pressure 3 times from $50 \mathrm{~mL}$ of $\mathrm{CH}_{2} \mathrm{Cl}_{2}$ and then used directly in the next reaction.

To the crude residue from the previous step is added $300 \mathrm{~mL}$ of $\mathrm{CH}_{2} \mathrm{Cl}_{2}$ and stirred at $21^{\circ} \mathrm{C}$ until all solids dissolve. To the resulting solution is added chloranil (4.56 g, $18.53 \mathrm{mmol}, 1.05$ equiv) and the reaction is allowed to stir $2 \mathrm{~h}$ at $21^{\circ} \mathrm{C}$. The reaction is then filtered through a silica pad, washed with $\mathrm{CH}_{2} \mathrm{Cl}_{2}$, and evaporated under reduced pressure. The crude residue was then suspended in $\mathrm{Et}_{2} \mathrm{O}(75 \mathrm{~mL})$ and stirred for $3 \mathrm{~h}$ at $21^{\circ} \mathrm{C}$. The suspension was then filtered, and the solids washed with $3 \times 25 \mathrm{~mL}$ $\mathrm{Et}_{2} \mathrm{O}$ washings to yield tosylate SI-15 (5.75 g, 84\% yield) as a light brown solid. ${ }^{1} \mathrm{H}$ NMR (400 MHz, $\left.\mathrm{CDCl}_{3}\right): \delta 8.08(\mathrm{~d}, J=8.0,1 \mathrm{H}), 7.92(\mathrm{~d}, J=8.0,1 \mathrm{H}), 7.81(\mathrm{~d}, J=8.0,1 \mathrm{H}), 7.56-7.52(\mathrm{~m}, 1 \mathrm{H}), 7.50$ $7.46(\mathrm{~m}, 1 \mathrm{H}), 7.34(\mathrm{~d}, J=8.0,1 \mathrm{H}), 5.12-5.05(\mathrm{~m}, 1 \mathrm{H}), 4.27-4.19(\mathrm{~m}, 2 \mathrm{H}), 3.73-3.65(\mathrm{dd}, J=20.0$, 12.0, 1H), 3.54-3.48 (dd, $J=16.0,8.0,1 \mathrm{H}), 2.43$ (s, 3H). ${ }^{13} \mathrm{C}$ NMR (100 MHz, $\left.\mathrm{CDCl}_{3}\right): \delta 157.0,154.4$, 153.1, 145.3, 135.1, 132.5, 130.0, 128.0, 126.8, 126.6, 123.9, 121.9, 79.4, 69.0, 36.7, 21.7; HRMS-ESI $(\mathrm{m} / \mathrm{z})[\mathrm{M}+\mathrm{Na}]^{+}$calculated for $\mathrm{C}_{18} \mathrm{H}_{16} \mathrm{O}_{4} \mathrm{~N}_{2} \mathrm{~S}_{2} \mathrm{Na}$, 411.04437; found, 411.04529 . 


\section{Synthesis Of Cyclopropanes}

Cyclopropanes were synthesized following the general procedure below.
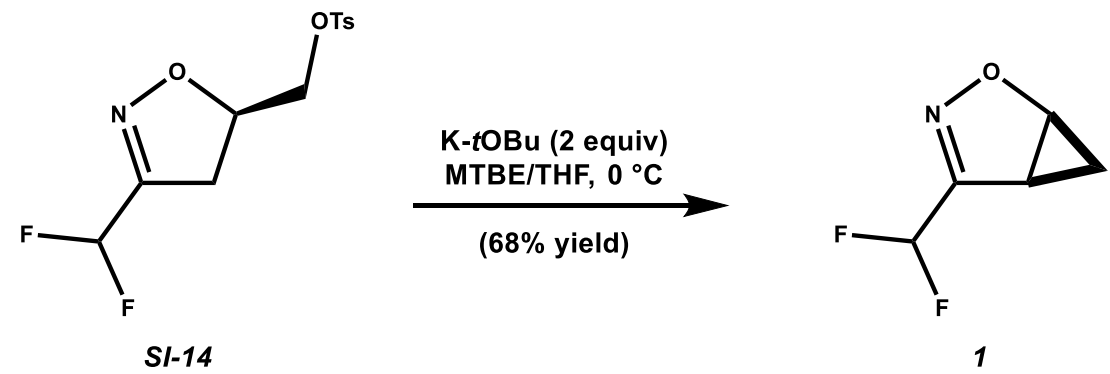

Cyclopropane 1. A round-bottom flask was charged with tosylate SI-14 (1.10 g, $3.60 \mathrm{mmol}, 1$ equiv), followed by the addition of MTBE (36 mL) and THF (36 mL). The resulting solution was then cooled to $0{ }^{\circ} \mathrm{C}$ and potassium tert-butoxide ( $811 \mathrm{mg}, 7.23 \mathrm{mmol}, 2$ equiv) was added. The reaction temperature was maintained at $0{ }^{\circ} \mathrm{C}$ while the mixture was stirred for $30 \mathrm{~min}$. The reaction was then quenched by the slow addition of saturated aqueous $\mathrm{NH}_{4} \mathrm{Cl}$ solution $(10 \mathrm{~mL})$. The mixture was allowed to warm to room temperature and was then transferred to a separatory funnel with EtOAc $(50 \mathrm{~mL})$ and water $(20 \mathrm{~mL})$. The resulting biphasic mixture was extracted with EtOAc $(2 \times 50 \mathrm{~mL})$ and the organic layers were combined, dried over $\mathrm{MgSO}_{4}$, filtered, and evaporated under reduced pressure. The crude residue was purified by automated flash chromatography ( $80 \mathrm{~g}$ column, $100 \%$ hexanes to $60 \%$ hexanes / $40 \%$ EtOAc gradient over $30 \mathrm{~min})$ to yield cyclopropane 1 ( $0.326 \mathrm{~g}, 68 \%$ yield, $99.72 \%$ ee, yellow oil. ${ }^{1} \mathrm{H}$ NMR (400 $\left.\mathrm{MHz}, \mathrm{CDCl}_{3}\right): 6.44\left(\mathrm{t}, J_{H-F}=56.0,1 \mathrm{H}\right), 5.11-5.07(\mathrm{~m}, 1 \mathrm{H}), 4.16-4.08(\mathrm{~m}, 2 \mathrm{H}), 2.81-32.77(\mathrm{~m}, 1 \mathrm{H})$, 1.12-1.07 (m, 1H), 0.41-0.38 (m, 1H); ${ }^{13} \mathrm{C}$ NMR (100 MHz, $\left.\mathrm{CDCl}_{3}\right): \delta 157.7\left(\mathrm{t}, J_{C^{-} F}=31.0\right), 109.4(\mathrm{t}$, $\left.J_{C-F}=234.0\right), 65.5,23.0,6.4 ;{ }^{19} \mathrm{~F}$ NMR $\left({ }^{1} \mathrm{H}\right.$ decoupled $)\left(377 \mathrm{MHz}, \mathrm{CDCl}_{3}\right):-115.3\left(\mathrm{~d}, J_{F-F}=324.2\right.$, 1F), $-121.9\left(\mathrm{~d}, J_{F^{-} F}=324.2,1\right.$ FHRMS-ESI $(\mathrm{m} / \mathrm{z})[\mathrm{M}+\mathrm{H}]^{+}$calculated for $\mathrm{C}_{5} \mathrm{H}_{6} \mathrm{ONF}_{2}, 134.04120$; found, 134.04181 .

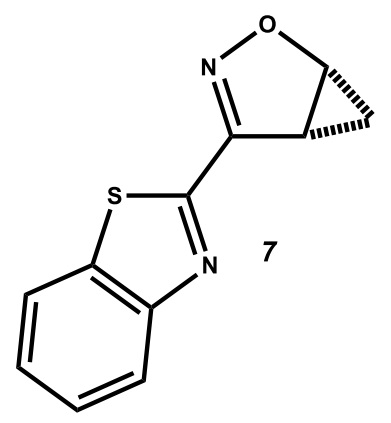


Cyclopropane 7. 78\% yield, $99.92 \% e e$, white solid. ${ }^{1} \mathrm{H}$ NMR (400 MHz, $\left.\mathrm{CDCl}_{3}\right): \delta 8.13$ (d, $J=8.0$, 1H), $7.92(\mathrm{~d}, J=8.0,1 \mathrm{H}), 7.56-7.52(\mathrm{~m}, 1 \mathrm{H}), 7.49-7.45(\mathrm{~m}, 1 \mathrm{H}), 5.22-5.19(\mathrm{~m}, 1 \mathrm{H}), 3.36-3.32(\mathrm{~m}$, 1H), 1.25-1.20 (m, 1H), 0.59-0.56 (m, 1H). $\left.{ }^{13} \mathrm{C} \mathrm{NMR} \mathrm{(100} \mathrm{MHz,} \mathrm{CDCl}_{3}\right): \delta 159.2,157.4,153.2,135.2$, 126.7, 126.6, 123.9, 121.9, 66.3, 25.7, 7.5; HRMS-ESI $(\mathrm{m} / \mathrm{z})[\mathrm{M}+\mathrm{H}]^{+}$calculated for $\mathrm{C}_{11} \mathrm{H}_{9} \mathrm{ON}_{2} \mathrm{~S}$, 217.04301; found, 217.04341.

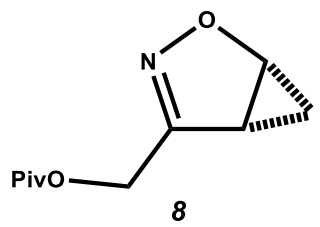

Cyclopropane 8. 71\% yield, 99.08 ee, yellow oil. ${ }^{1} \mathrm{H}$ NMR (400 MHz, $\left.\mathrm{CDCl}_{3}\right)$ : $\delta 4.98-4.86(\mathrm{~m}, 3 \mathrm{H})$, 2.60-2.55 (m, 1H), 1.25 (s, 9H), 0.97-0.95 (m, 1H), 0.32-0.29 (m, 1H); ${ }^{13} \mathrm{C}$ NMR (100 MHz, $\left.\mathrm{CDCl}_{3}\right)$ : $\delta$ 177.9, 159.8, 64.2, 58.4, 38.9, 27.1, 26.2, 7.0; HRMS-ESI $(\mathrm{m} / \mathrm{z})[\mathrm{M}+\mathrm{H}]^{+}$calculated for $\mathrm{C}_{10} \mathrm{H}_{16} \mathrm{O}_{3} \mathrm{~N}$, 198.11247; found, 198.11287.

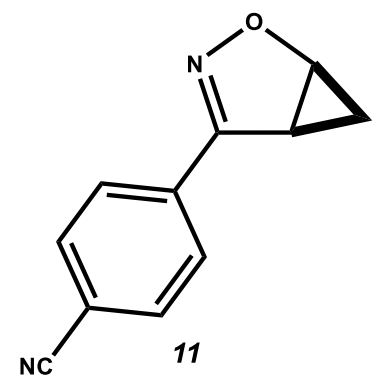

Cyclopropane 11. 80\% yield, $96.50 \% e e$, white solid. ${ }^{1} \mathrm{H}$ NMR (400 MHz, $\left.\mathrm{CDCl}_{3}\right): \delta 7.91-7.89(\mathrm{~m}, 2 \mathrm{H})$, 7.73-7.71 (m, 2H), 5.14-5.11 (m, 1H), 2.91-2.87 (m, 1H), 1.17-1.12 (m, 1H), 0.48-0.45 (m, 1H); ${ }^{13} \mathrm{C}$ NMR (100 MHz, $\left.\mathrm{CDCl}_{3}\right): \delta 160.4,133.2,132.4,127.5,118.3,113.6,65.1,25.1,7.5 ;$ HRMS-ESI (m/z) $[\mathrm{M}+\mathrm{H}]^{+}$calculated for $\mathrm{C}_{11} \mathrm{H}_{9} \mathrm{~N}_{2} \mathrm{O}, 185.0709$; found, 185.0710.

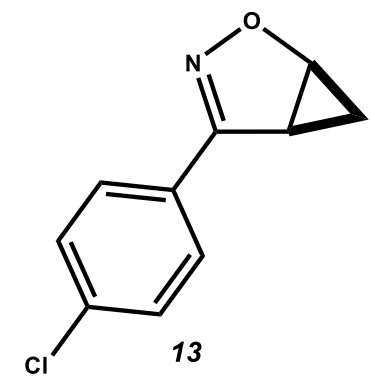


Cyclopropane 13. 86\% yield, $98.72 \%$ ee, white solid. ${ }^{1} \mathrm{H}$ NMR (400 MHz, $\left.\mathrm{CDCl}_{3}\right)$ : $\delta 7.75-7.72(\mathrm{~m}, 2 \mathrm{H})$, 7.43-7.39 (m, 2H), 5.08-5.05 (m, 1H), 2.88-2.84 (m, 1H), 1.12-1.07 (m, 1H), 0.47-0.44 (m, 1H); ${ }^{13} \mathrm{C}$ NMR (100 MHz, $\left.\mathrm{CDCl}_{3}\right): \delta 160.9,136.3,129.0,128.4,127.5,64.5,25.6,7.7 ;$ HRMS-ESI (m/z) [M + $\mathrm{H}]^{+}$calculated for $\mathrm{C}_{10} \mathrm{H}_{9} \mathrm{NOCl}, 194.03672$; found, 194.03688 .

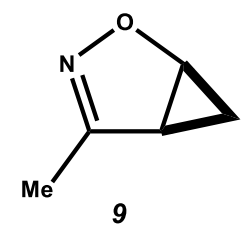

Cyclopropane 9. 53\% yield, 98.98\% ee, yellow oil. ${ }^{1} \mathrm{H}$ NMR (400 MHz, $\left.\mathrm{CDCl}_{3}\right): \delta 4.86-4.83(\mathrm{~m}, 1 \mathrm{H})$, 2.46-2.42 (m, 1H), 2.07 (s, 3H), 0.90-0.84 (m, 1H), 0.30-0.27 (m, 1H); $\left.{ }^{13} \mathrm{C} \mathrm{NMR} \mathrm{(100} \mathrm{MHz,} \mathrm{CDCl}_{3}\right)$ : $\delta$ 160.3, 63.5, 28.7, 12.8, 7.5; HRMS-ESI $(\mathrm{m} / \mathrm{z})[\mathrm{M}+\mathrm{H}]^{+}$calculated for $\mathrm{C}_{5} \mathrm{H}_{7} \mathrm{ON}, 98.0600$; found, 98.0605 .

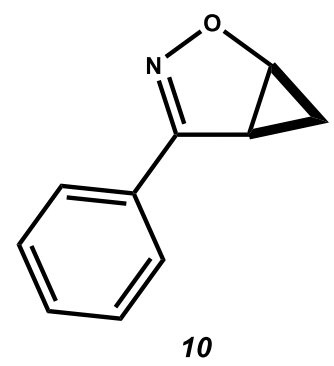

Cyclopropane 10. 88\% yield, $94.54 \% e e$ ) as a white solid. ${ }^{1} \mathrm{H} \mathrm{NMR}\left(400 \mathrm{MHz}, \mathrm{CDCl}_{3}\right)$ : $\delta 7.83-7.78(\mathrm{~m}$, 2H), 7.47-7.41 (m, 3H), 5.06-5.03 (m, 1H), 2.92-2.87 (m, 1H), 1.11-1.06 (m, 1H), 0.47-0.44 (m, 1H); ${ }^{13} \mathrm{C} \mathrm{NMR}\left(100 \mathrm{MHz}, \mathrm{CDCl}_{3}\right): \delta 161.7,130.2,129.0,128.7,127.1,64.2,25.7,7.7 ; \mathrm{HRMS}-\mathrm{ESI}(\mathrm{m} / \mathrm{z})[\mathrm{M}$ $+\mathrm{H}]^{+}$calculated for $\mathrm{C}_{10} \mathrm{H}_{10} \mathrm{ON}, 160.07569$; found, 160.07608 .

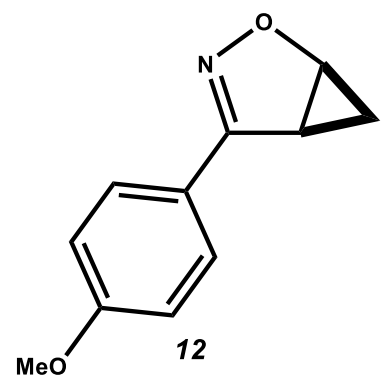

Cyclopropane 12. $82 \%$ yield, $100 \%$ ee, white solid. ${ }^{1} \mathrm{H}$ NMR (400 MHz, $\left.\mathrm{CDCl}_{3}\right): \delta 7.76-7.74(\mathrm{~m}, 2 \mathrm{H})$, 6.98-6.95 (m, 2H), 5.02-4.99 (m, 1H), 3.87 (s, 3H), 2.87-2.83 (m, 1H), 1.08-1.02 (m, 1H), 0.46-0.43 
(m, 1H); ${ }^{13} \mathrm{C}$ NMR (100 MHz, $\left.\mathrm{CDCl}_{3}\right): \delta 161.3,128.8,121.7 .0,120.1,114.1,63.9,55.4,26.0,8.0$; HRMS-ESI $(\mathrm{m} / \mathrm{z})[\mathrm{M}+\mathrm{H}]^{+}$calculated for $\mathrm{C}_{11} \mathrm{H}_{12} \mathrm{NO}_{2}, 190.0863$; found, 190.0861 .

\section{E. Racemization Studies Of Cyclopropanes}

Racemization of cyclopropanes was conducted on $0.05 \mathrm{M}$ solutions following the general procedure below in the appropriate solvent and temperature listed in the tables.
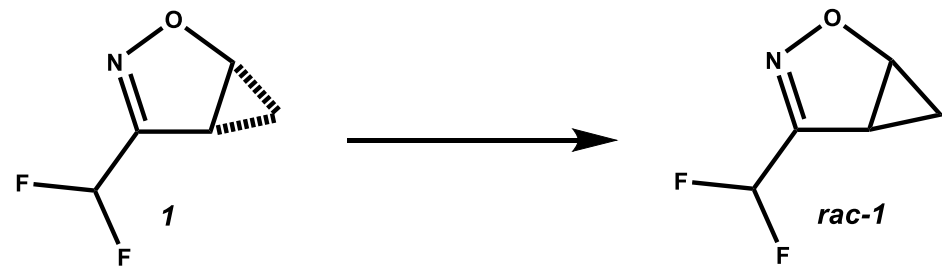

A 1-dram vial was charged with cyclopropane $1(13.3 \mathrm{mg}, 0.100 \mathrm{mmol})$, followed by the addition of $\mathrm{CH}_{3} \mathrm{CN}(2 \mathrm{~mL})$. The resulting solution was then stirred and maintained at $30{ }^{\circ} \mathrm{C}$ in an oil bath. Samples were pulled periodically for HPLC analysis to determine $e e$.

\begin{tabular}{|c|c|c|c|c|c|c|c|}
\hline \multirow[t]{2}{*}{ Time (h) } & \multicolumn{3}{|c|}{ ee In Various Solvents At $30^{\circ} \mathrm{C}$} & \multirow[t]{2}{*}{ Time (h) } & \multicolumn{3}{|c|}{ ee In Various Solvents At $45^{\circ} \mathrm{C}$} \\
\hline & $\underline{M T B E}$ & Acetone & Acetonitrile & & $\underline{M T B E}$ & Acetone & $\underline{\text { Acetonitrile }}$ \\
\hline 0 & 99.72 & 99.72 & 99.72 & 0 & 99.72 & 99.72 & 99.72 \\
\hline 24 & 92.3 & 77.38 & 67 & 24 & 60.18 & 20.3 & 7.3 \\
\hline 48 & 85.36 & 60.72 & 46.32 & 48 & 37.06 & 4.74 & 0.4 \\
\hline 72 & 79.24 & 46.82 & 30.94 & 72 & 22.24 & 1.38 & 0.06 \\
\hline 96 & 73.26 & 36.5 & 21.18 & 96 & 13.46 & 0.7 & 0.08 \\
\hline 120 & 67.6 & 28.3 & 14.12 & 120 & 7.9 & 0.66 & 0.06 \\
\hline
\end{tabular}

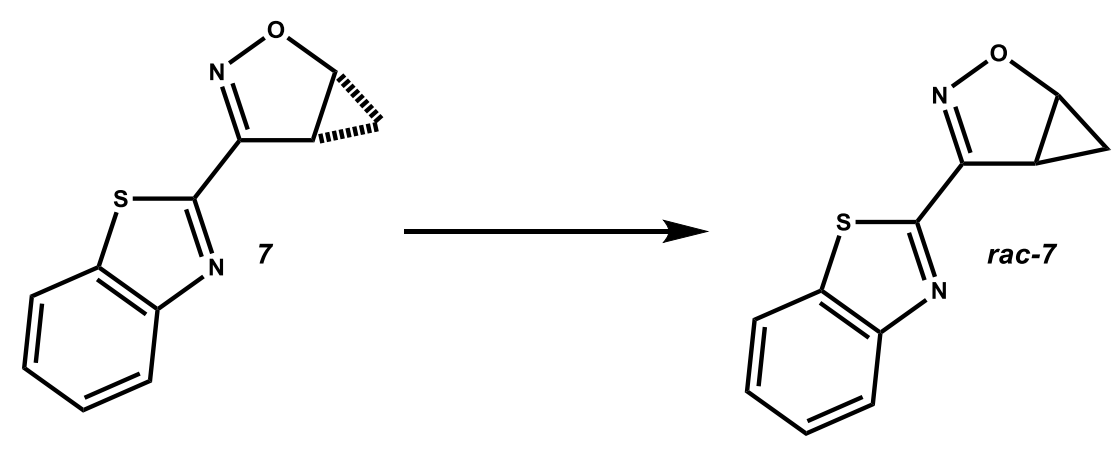




\begin{tabular}{|c|c|c|c|c|c|c|}
\hline Time (h) & \multicolumn{9}{|c|}{ ee In Various Solvents At $\mathbf{3 0}^{\circ} \mathbf{C}$} & & Time (h) & \\
\hline & MTBE & Toluene & Acetonitrile & & \\
\hline 0 & 99.92 & 99.92 & 99.92 & & & Acetone \\
\hline 24 & 98.32 & 97.64 & 93.48 & 25 & 99.92 \\
\hline 48 & 96.1 & 93.18 & 81.34 & 55 & 87.48 \\
\hline 73 & 94.32 & 90.32 & 73.26 & 72 & 84.1 \\
\hline 103 & 92.1 & 87.2 & 66.4 & & 96 & 80.46 \\
\hline 120 & 90.7 & 83.8 & 63.34 & & 120 & 76.56 \\
\hline
\end{tabular}

\begin{tabular}{|c|c|c|c|c|c|c|}
\hline Time (h) & \multicolumn{3}{|c|}{$\boldsymbol{e e}$ In Various Solvents At 45 ${ }^{\circ} \mathbf{C}$} & & Time (h) & \\
\hline & MTBE & Toluene & Acetonitrile & & & Acetone \\
\hline 0 & 99.92 & 99.92 & 99.92 & & 0 & 99.92 \\
\hline 24 & 85 & 71.42 & 47.3 & & 25 & 64.34 \\
\hline 48 & 70.58 & 51.58 & 16.36 & 75 & 40.42 \\
\hline 73 & 60.46 & 40.38 & 9.48 & & 72 & 29.48 \\
\hline 103 & 51.58 & 31.44 & 5.14 & & 96 & 20.18 \\
\hline 120 & 46.64 & 27.16 & 4.08 & & 120 & 14.4 \\
\hline
\end{tabular}
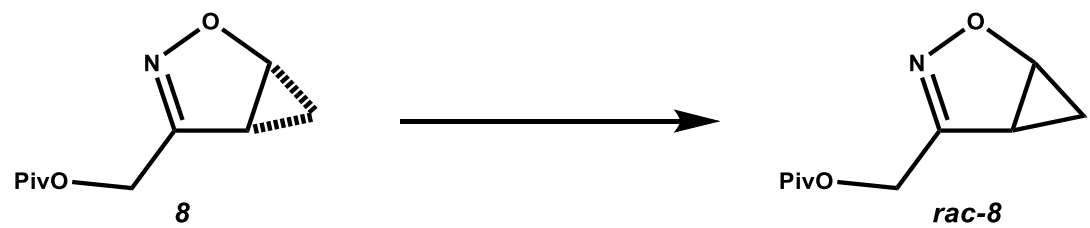

\begin{tabular}{|c|c|c|c|c|c|c|c|}
\hline \multirow[t]{2}{*}{ Time (h) } & \multicolumn{3}{|c|}{ ee In Various Solvents At $30^{\circ} \mathrm{C}$} & \multirow[t]{2}{*}{ Time (h) } & \multicolumn{3}{|c|}{ ee In Various Solvents At $45^{\circ} \mathrm{C}$} \\
\hline & $\underline{M T B E}$ & Acetone & Acetonitrile & & $\underline{M T B E}$ & Acetone & Acetonitrile \\
\hline 0 & 99.08 & 99.08 & 99.08 & 0 & 99.08 & 99.08 & 99.08 \\
\hline 24 & 98.26 & 97.94 & 97.86 & 24 & 94.34 & 91.86 & 90.34 \\
\hline 48 & 97.58 & 97.18 & 96.96 & 48 & 90.42 & 85.76 & 82.88 \\
\hline 72 & 97.1 & 96.42 & 96.02 & 72 & 86.2 & 79.5 & 75.58 \\
\hline 96 & 96.66 & 95.6 & 95.02 & 96 & 81.96 & 73.46 & 68.5 \\
\hline 120 & 96.12 & 94.8 & 94.06 & 120 & 78 & 68.16 & 62.62 \\
\hline 144 & 95.28 & 93.96 & 93.06 & 144 & 73.72 & 62.62 & 56.9 \\
\hline 168 & 94.86 & 93.26 & 92.16 & 168 & 70.1 & 57.72 & 51.7 \\
\hline
\end{tabular}

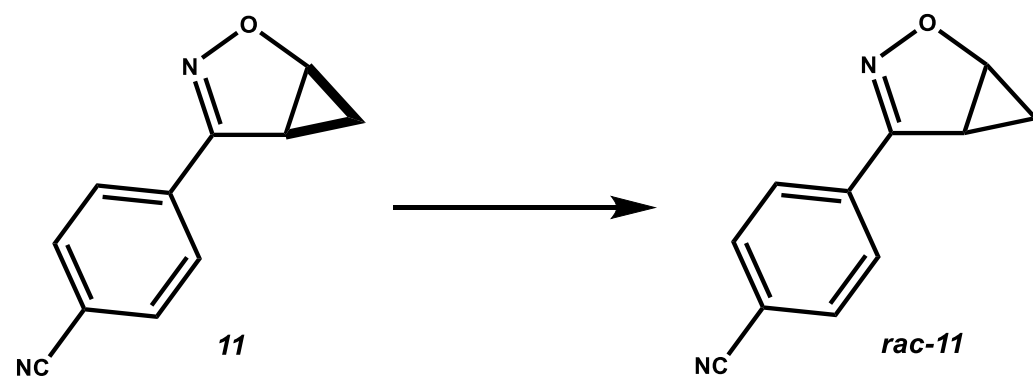




\begin{tabular}{|c|c|c|}
\hline Time (h) & \multicolumn{2}{|c|}{ ee In Acetonitrile At $70^{\circ} \mathbf{C}$} \\
\hline & Acetonitrile & \\
\hline 0 & 96.5 & \\
\hline 24 & 51.38 & \\
\hline 48 & 28.92 & \\
\hline 72 & 14.76 & \\
\hline
\end{tabular}

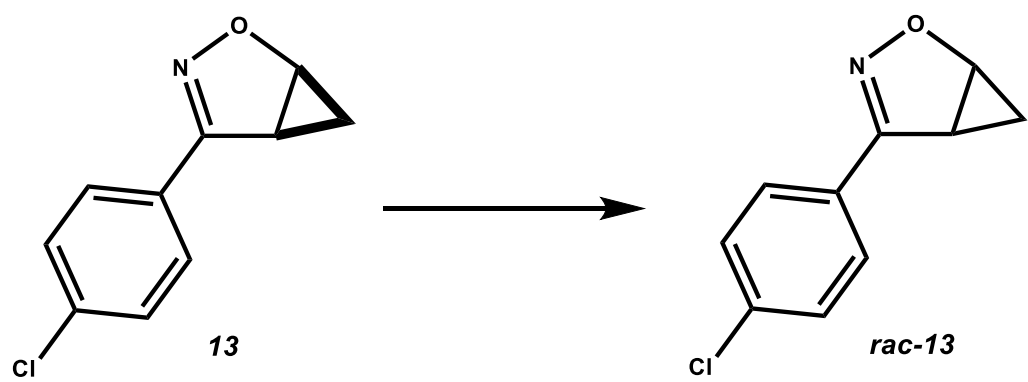

Time (h) Time (s) ee In Acetonitrile At $70^{\circ} \mathrm{C}$

\begin{tabular}{|c|c|c|c|}
\hline & & & New Acetonitrile \\
\hline 0 & 0 & 98.72 \\
\hline 24 & 86400 & 62.84 \\
\hline 48 & 172800 & 43.64 \\
\hline 72 & 259200 & 30.44 \\
\hline
\end{tabular}

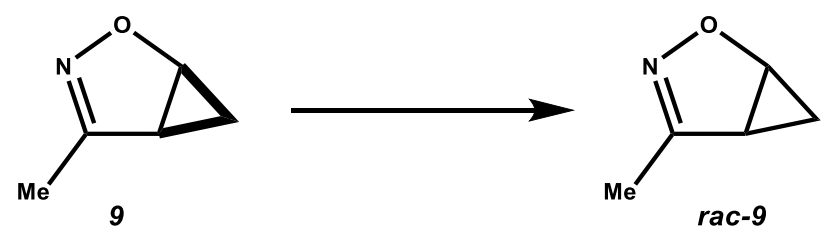

\begin{tabular}{|c|c|c|c|c|c|c|c|}
\hline \multirow[t]{2}{*}{ Time (h) } & \multicolumn{3}{|c|}{ ee In Various Solvents At $30^{\circ} \mathrm{C}$} & \multirow[t]{2}{*}{ Time (h) } & \multicolumn{3}{|c|}{ ee In Various Solvents At $45^{\circ} \mathrm{C}$} \\
\hline & MTBE & Acetone & Acetonitrile & & $\underline{M T B E}$ & Acetone & Acetonitrile \\
\hline 0 & 98.9 & 98.9 & 98.9 & 0 & 98.9 & 98.9 & 98.9 \\
\hline 24 & 98.42 & 98.56 & 98.18 & 24 & 97.96 & 98.18 & 97.5 \\
\hline 48 & $N / A$ & N/A & N/A & 48 & $N / A$ & N/A & N/A \\
\hline 74 & 98.62 & 98.58 & 98.5 & 74 & 95.48 & 95.8 & N/A \\
\hline 98 & 98.94 & 98.72 & 98.3 & 98 & 94.94 & 94.52 & N/A \\
\hline 128 & 98.92 & 99.32 & 98 & 128 & 92.26 & 92.74 & N/A \\
\hline 171 & 98.48 & 98.4 & 98.72 & 171 & 91.12 & 91.52 & N/A \\
\hline
\end{tabular}

\begin{tabular}{|c|c|c|}
\hline Time (h) & \multicolumn{2}{|c|}{$\begin{array}{c}\text { ee In Acetonitrile At } \mathbf{7 0} \\
\text { Acetonitrile }\end{array}$} \\
\hline & \multicolumn{1}{c|}{$\mathbf{C}$} \\
\hline 0 & 98.98 & \\
\hline 24 & 82.26 & \\
\hline 49 & 66.72 & \\
\hline 72 & 54.32 & \\
\hline 144 & 28.54 & \\
\hline
\end{tabular}




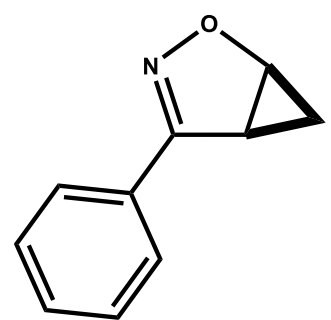

10

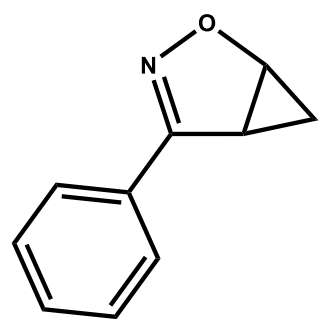

rac-10

\begin{tabular}{|c|c|c|c|c|c|c|c|}
\hline \multirow[t]{2}{*}{ Time (h) } & \multicolumn{3}{|c|}{ ee In Various Solvents At $30^{\circ} \mathrm{C}$} & \multirow[t]{2}{*}{ Time (h) } & \multicolumn{3}{|c|}{ ee In Various Solvents At $45^{\circ} \mathrm{C}$} \\
\hline & MTBE & Acetone & Acetonitrile & & MTBE & Acetone & Acetonitrile \\
\hline 0 & 95.4 & 95.4 & 95.4 & 0 & 95.4 & 95.4 & 95.4 \\
\hline 24 & 95.02 & 94.98 & 95.74 & 24 & 94.84 & 94.24 & 94.7 \\
\hline 48 & 95.08 & 94.88 & 95.64 & 48 & 94.24 & 93.44 & 93.82 \\
\hline 72 & 95.04 & 94.8 & 95.44 & 72 & 93.9 & 92.76 & 92.84 \\
\hline 96 & 94.96 & 94.72 & 95.5 & 96 & 93.32 & 91.96 & 92.04 \\
\hline 120 & 94.92 & 94.66 & 95.34 & 120 & 92.8 & 91.12 & 91.14 \\
\hline
\end{tabular}

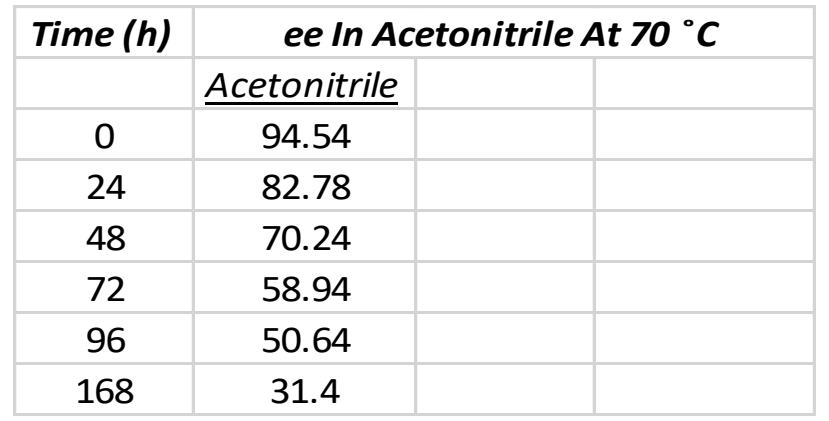

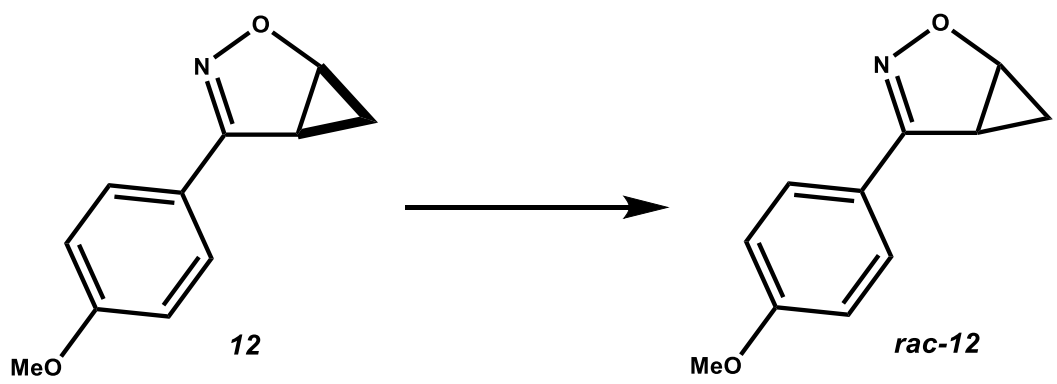

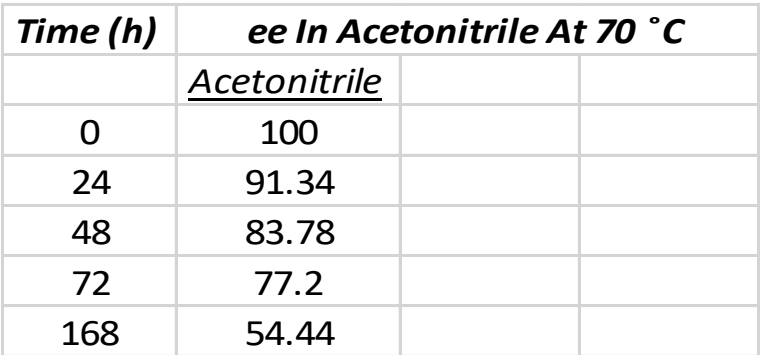


An example of for how experimental $\Delta \mathrm{G}^{\ddagger}$ values were calculated is shown below for cyclopropane $\mathbf{1}$ in acetonitrile at $30{ }^{\circ} \mathrm{C}$. Here $\mathrm{A}$ is the concentration of the starting cyclopropane $\mathbf{1}$ and $\mathrm{A} *$ is the concentration of ent-cyclopropane $\mathbf{1}$ as the racemization proceeds. $\mathrm{A}_{\mathrm{eq}}$ is the equilibrium concentration which is equal to half the initial concentration since this racemization is completely reversible. For these reactions the initial concentration is always $0.05 \mathrm{M}$ so $\mathrm{A}_{\text {eq }}$ is always $0.025 \mathrm{M}$. Given that this is a completely reversible first order reaction, a plot of $-\mathrm{LN}\left(\left[\mathrm{A}_{\mathrm{t}}\right]-\left[\mathrm{A}_{\mathrm{eq}}\right]\right)$ vs time is linear and the slope is $\left(k_{1}\right.$ $+k_{2}$ ). Given $k_{1}$ and $k_{2}$ are equal in this process the slope $/ 2=k$.

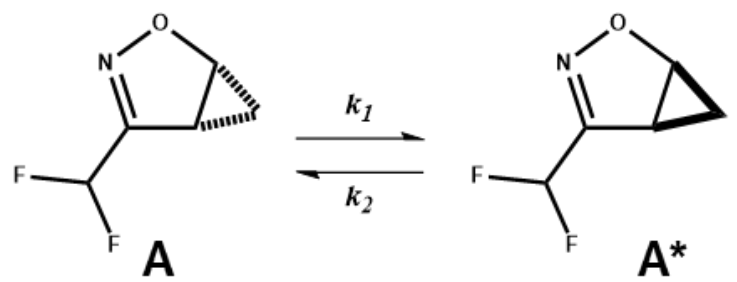

\begin{tabular}{|c|c|c|c|c|c|}
\hline Time (h) & Time $(\boldsymbol{s})$ & $\begin{array}{c}\text { ee In Various Solvents } \mathbf{A t} \mathbf{3 0}^{\circ} \mathbf{C} \\
\text { Acetonitrile }\end{array}$ & Concentration Of A & Concentration Of A* & LN A-Aeq \\
\hline 0 & 0 & 99.72 & 0.04993 & 0.00007 & 3.691683 \\
\hline 24 & 86400 & 67 & 0.04175 & 0.00825 & 4.089357 \\
\hline 48 & 172800 & 46.32 & 0.036575 & 0.01343 & 4.458908 \\
\hline 72 & 262800 & 30.94 & 0.032735 & 0.01727 & 4.862 \\
\hline 96 & 345600 & 21.18 & 0.030295 & 0.01971 & 5.240992 \\
\hline 120 & 432000 & 14.12 & 0.02853 & 0.02147 & 5.646457 \\
\hline
\end{tabular}

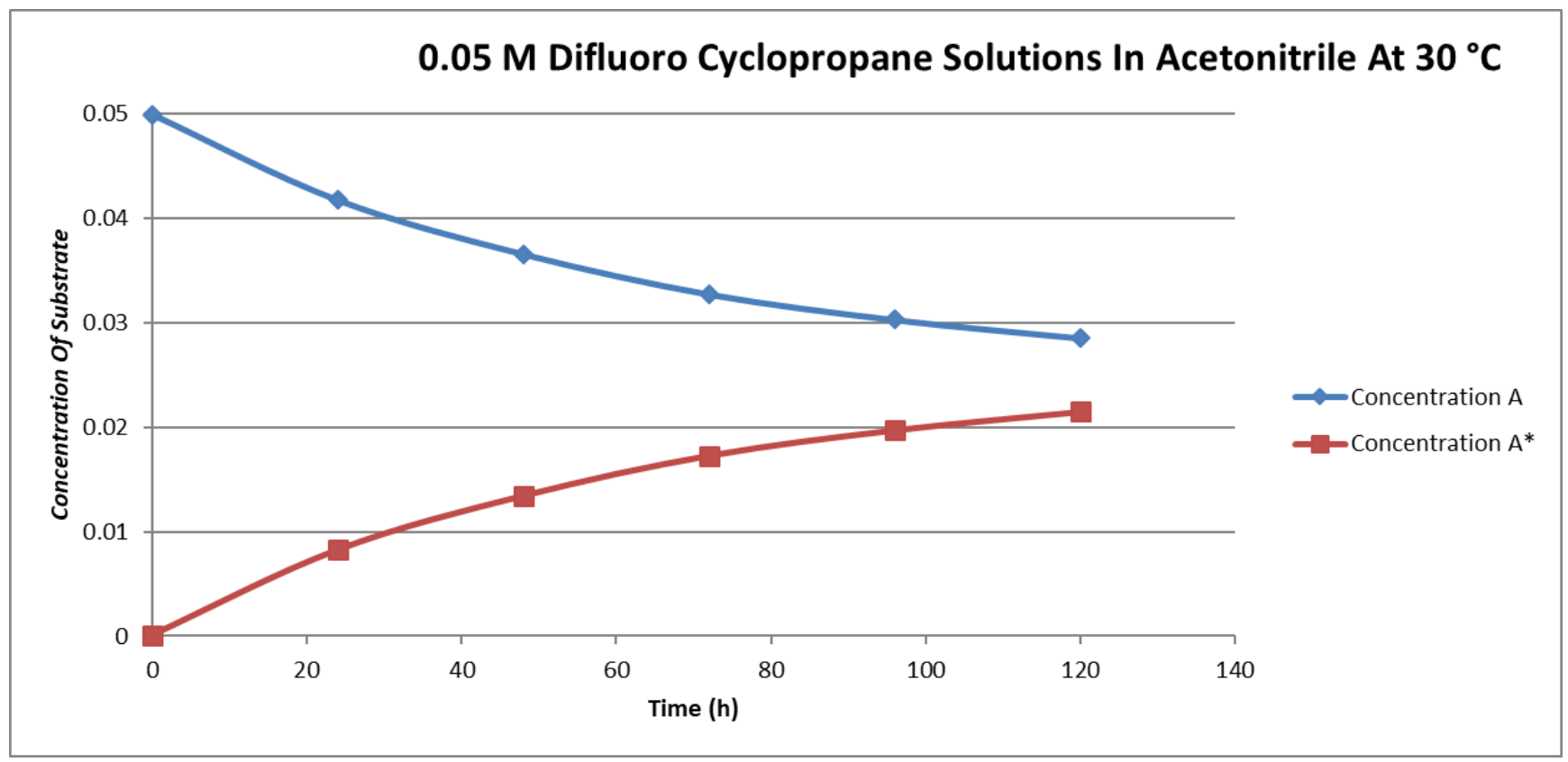




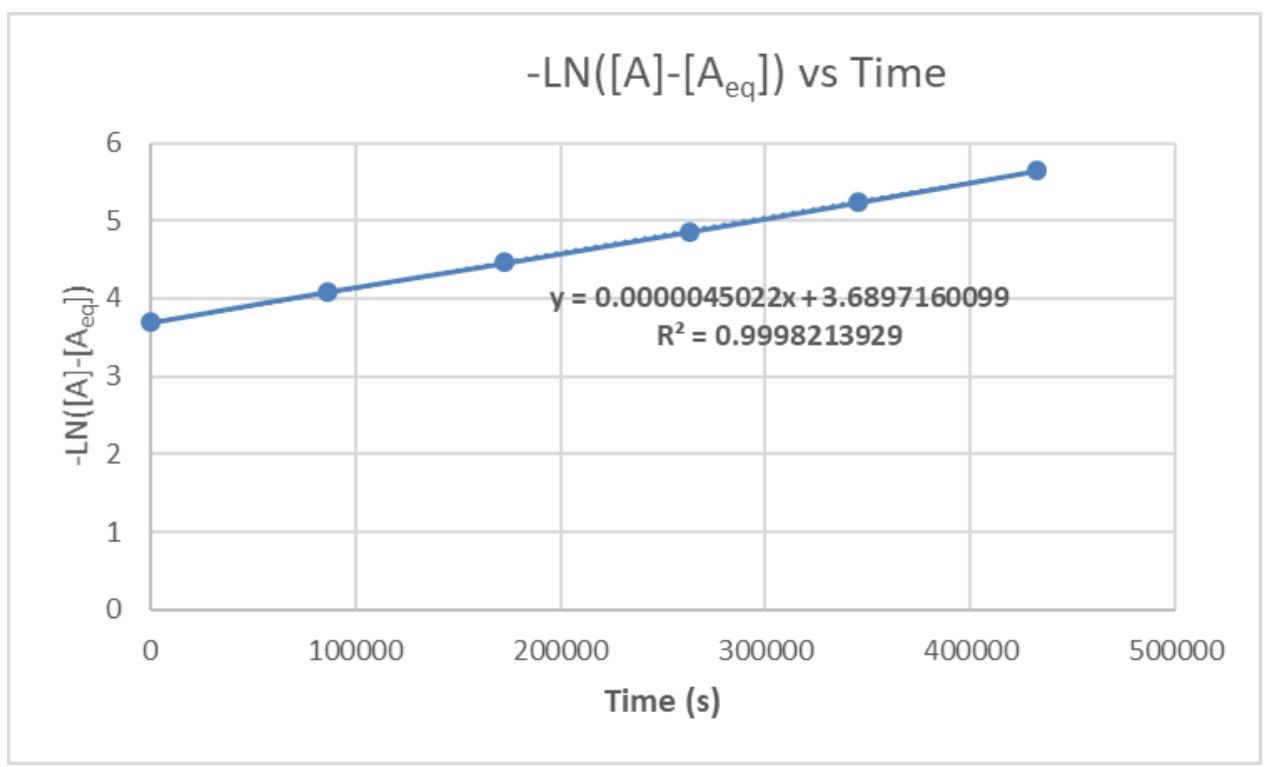

Determining $k$ from the plot above we can then use the flowing equation to solve for $\Delta \mathrm{G}^{\ddagger}$

$$
\Delta \mathrm{G}^{\dagger}=-\mathrm{RT} \ln \left(k h / k_{\mathrm{b}} \mathrm{T}\right)
$$

Converting the answer from this formula to $\mathrm{kcal} / \mathrm{mol}$ we get at $30{ }^{\circ} \mathrm{C} \Delta \mathrm{G}^{\ddagger}=25.6 \mathrm{kcal} / \mathrm{mol}$

The same calculation was done for all other cyclopropane compounds.

\section{Data For Hammett Plot Table}

The rate constants calculated from the above data tables and sigma values used to prepare the Hammett plot are shown here.

\begin{tabular}{|c|c|c|c|}
\hline & $\underline{\log (\boldsymbol{k} / \mathbf{k H})}$ & Sigma Value & rate constant $\boldsymbol{k}$ \\
\hline$p-\mathrm{CN}$ & 0.655278076 & 0.66 & 0.000005232 \\
\hline$p-\mathrm{Cl}$ & 0.289487174 & 0.23 & $2.2536 \mathrm{E}-06$ \\
\hline $\mathrm{H}$ & 0 & 0 & $1.15715 \mathrm{E}-06$ \\
\hline$p-\mathrm{OMe}$ & -0.191262535 & -0.27 & $7.4495 \mathrm{E}-07$ \\
\hline
\end{tabular}


Spectra And Chromatograms

\section{HPLC Chromatograms}


Compounds were separated on AS-H or AD-H chiral columns using 70/30 heptane/isopropanol mobile phases.

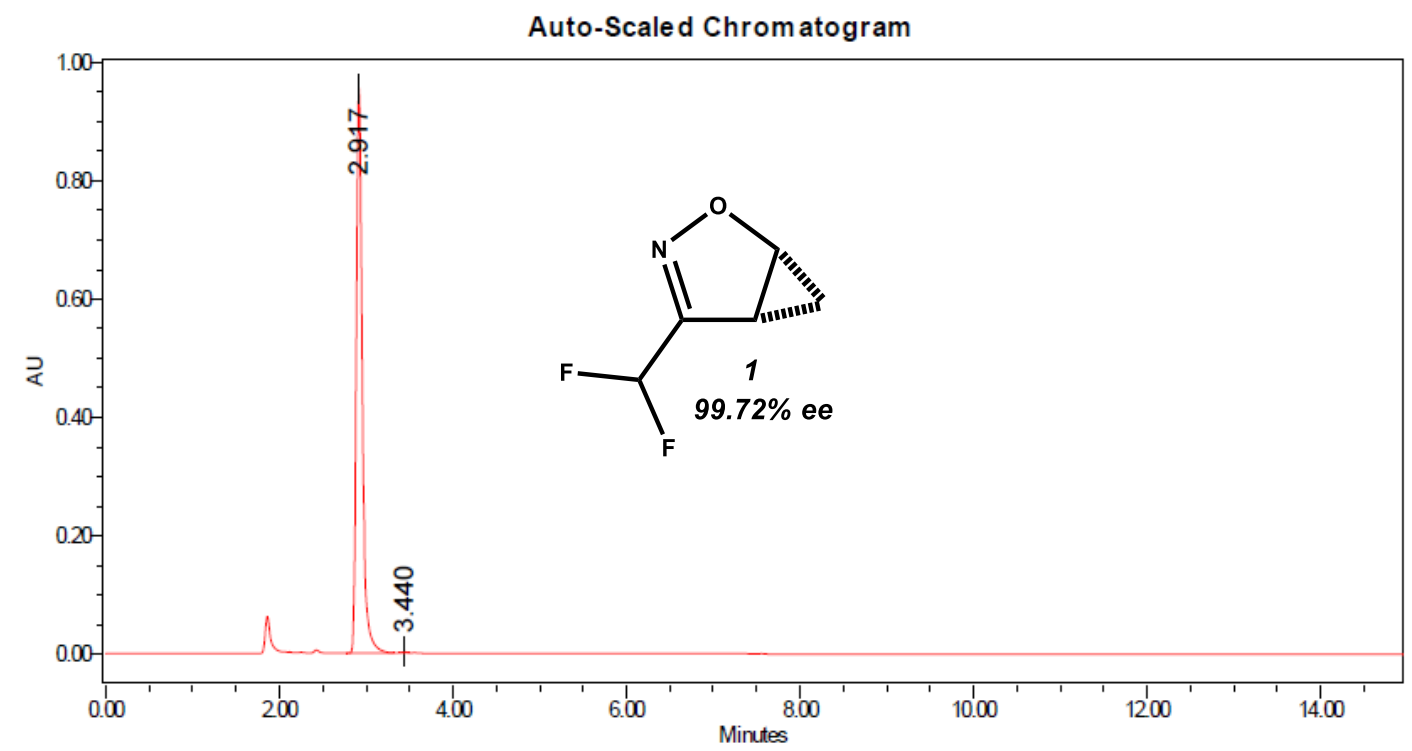

Processed Channel: DAD AU Ch 2 Sample

226, Bw 8

\begin{tabular}{|l|c|r|r|r|r|}
\hline & Processed Channel & $\begin{array}{r}\text { Retention } \\
\text { Time (min) }\end{array}$ & Area & $\%$ Area & Height \\
\hline 1 & DAD AU Ch2 Sample 226, Bw8 & 2917 & 4601474 & 99.86 & 954200 \\
\hline 2 & DAD AU Ch2 Sample 226, Bw8 & 3.440 & 6430 & 0.14 & 1737 \\
\hline
\end{tabular}




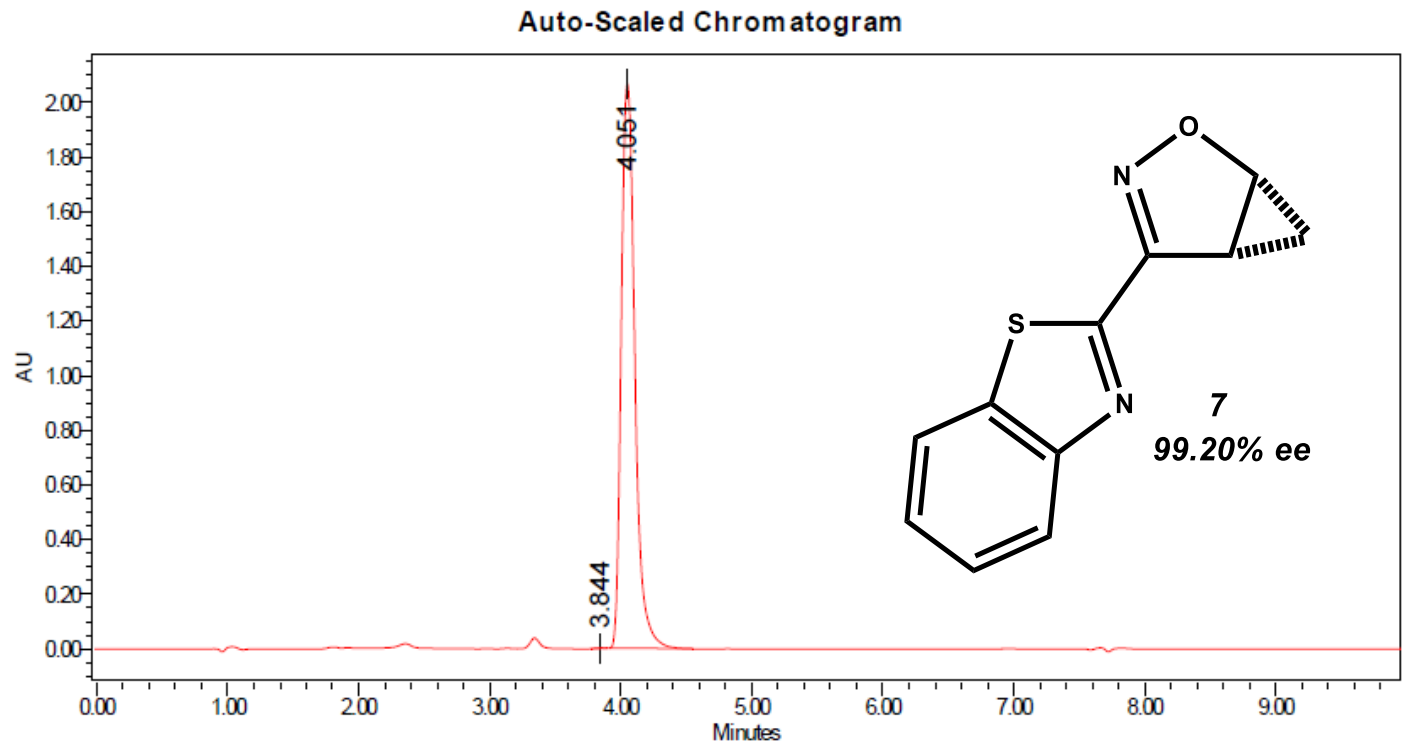

Processed Channel: DAD AU Ch 2 Sample

226, Bw 8

\begin{tabular}{|l|c|r|r|r|r|}
\hline & Processed Channel & $\begin{array}{r}\text { Retention } \\
\text { Time (min) }\end{array}$ & Area & \% Area & Height \\
\hline 1 & DAD AU Ch2 Sample 226, Bw8 & 3.844 & 6458 & 0.04 & 1700 \\
\hline 2 & DAD AU Ch2 Sample 226, Bw8 & 4.051 & 14902185 & 99.96 & 2067531 \\
\hline
\end{tabular}

Auto-Scaled Chrom atogram

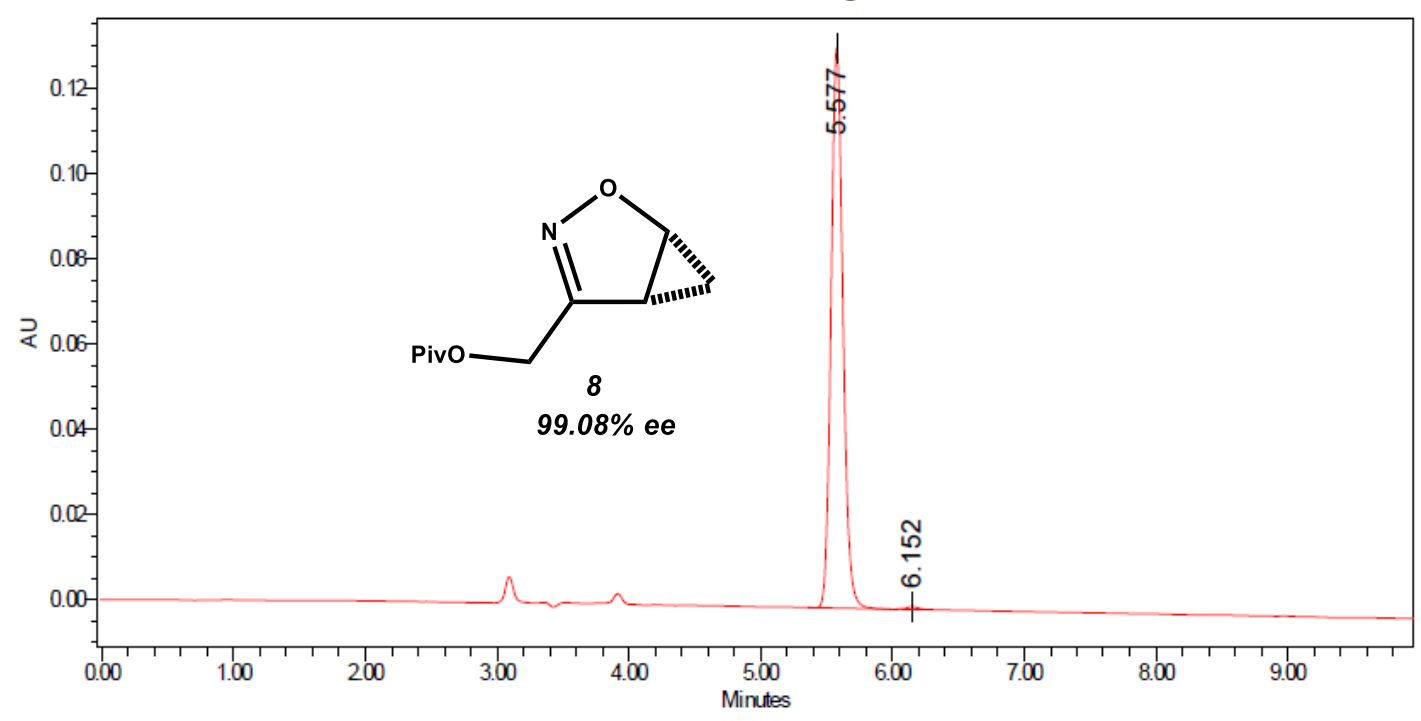

Processed Channel: DAD AU Ch 1 Sample

226, Bw 8

\begin{tabular}{|r|c|r|r|r|r|}
\hline & Processed Channel & $\begin{array}{c}\text { Retention } \\
\text { Time (min) }\end{array}$ & Area & $\%$ Area & Height \\
\hline 1 & DAD AU Ch1 Sample 226, Bw8 & 5.577 & 820489 & 99.54 & 131646 \\
\hline 2 & DAD AU Ch1 Sample 226, Bw8 & 6.152 & 3774 & 0.46 & 609 \\
\hline
\end{tabular}




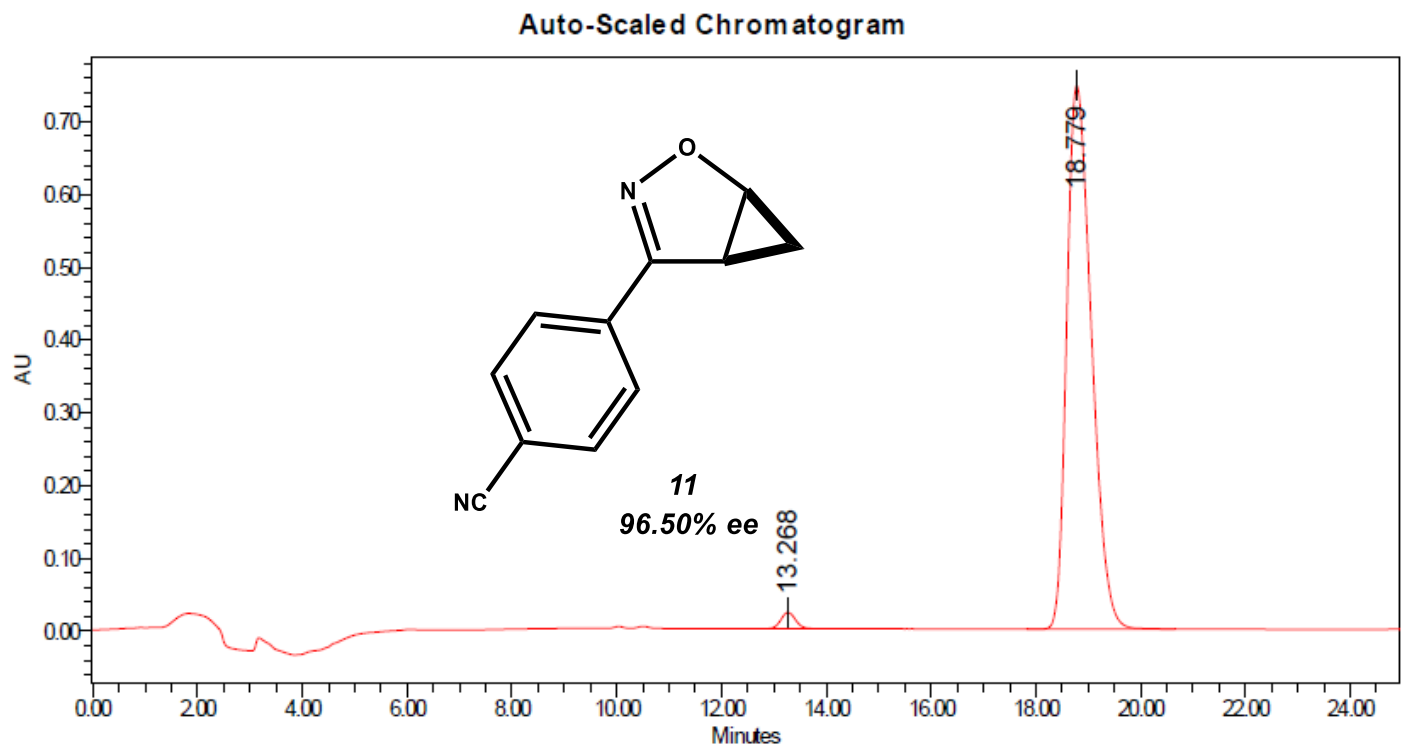

Processed Channel: DAD AU Ch 1 Sample

226, BW 8

\begin{tabular}{|l|c|r|r|r|r|}
\hline & Processed Charnel & $\begin{array}{c}\text { Retention } \\
\text { Time(min) }\end{array}$ & Area & \% Area & Height \\
\hline 1 & DAD AU Ch 1 Sample 226, Bw8 & 13.268 & 434788 & 1.75 & 22567 \\
\hline 2 & DAD AU Ch 1 Sample 226, Bw8 & 18.779 & 24383438 & 98.25 & 746832 \\
\hline
\end{tabular}

Auto-Scaled Chrom atogram

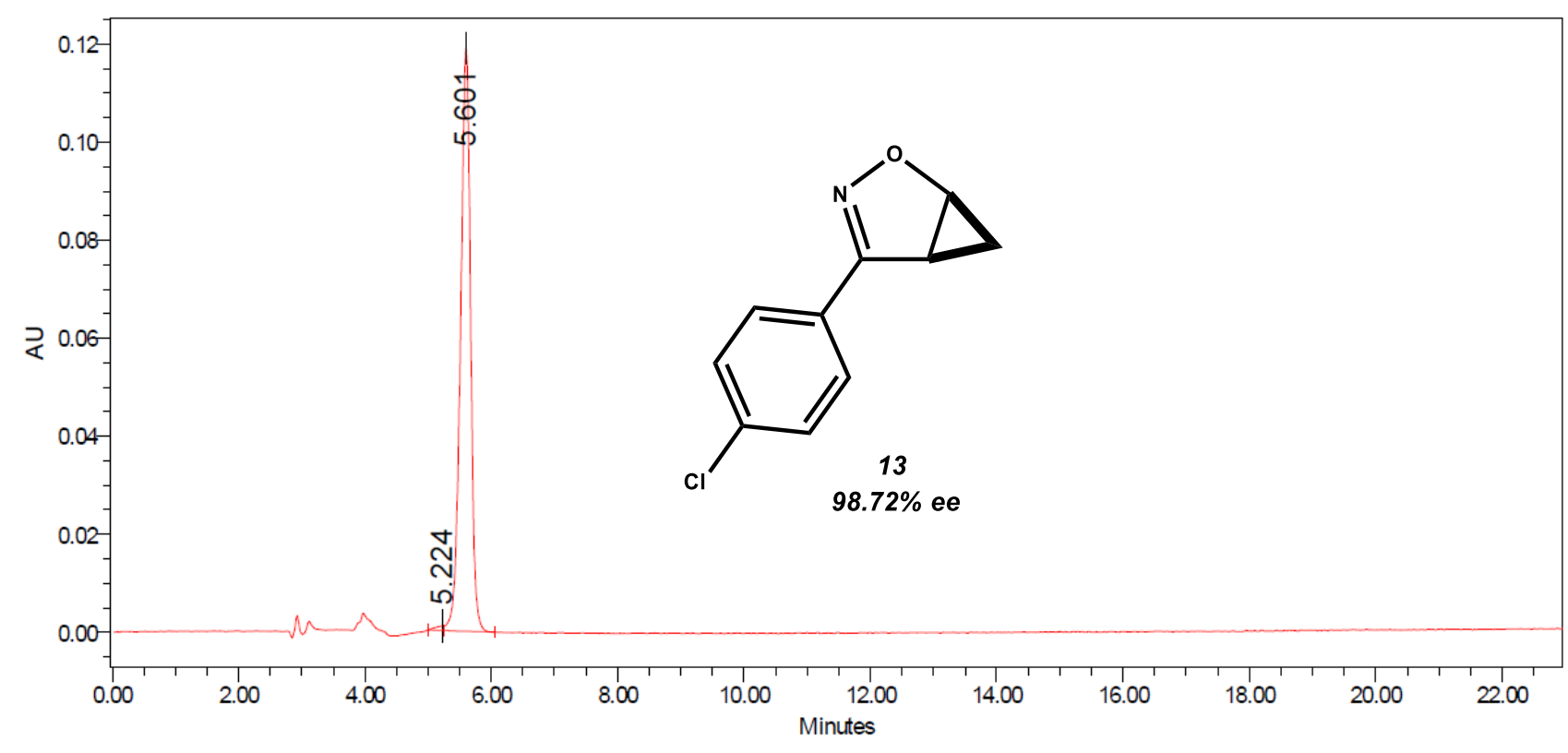

Peak Results

\begin{tabular}{|c|c|c|c|c|c|c|c|}
\hline & Name & RT & Area & $\%$ Area & Height & USP Tailing & RRT \\
\hline 1 & & 5.224 & 8419 & 0.64 & 904 & & \\
\hline 2 & & 5.601 & 1316194 & 99.36 & 119090 & & \\
\hline
\end{tabular}




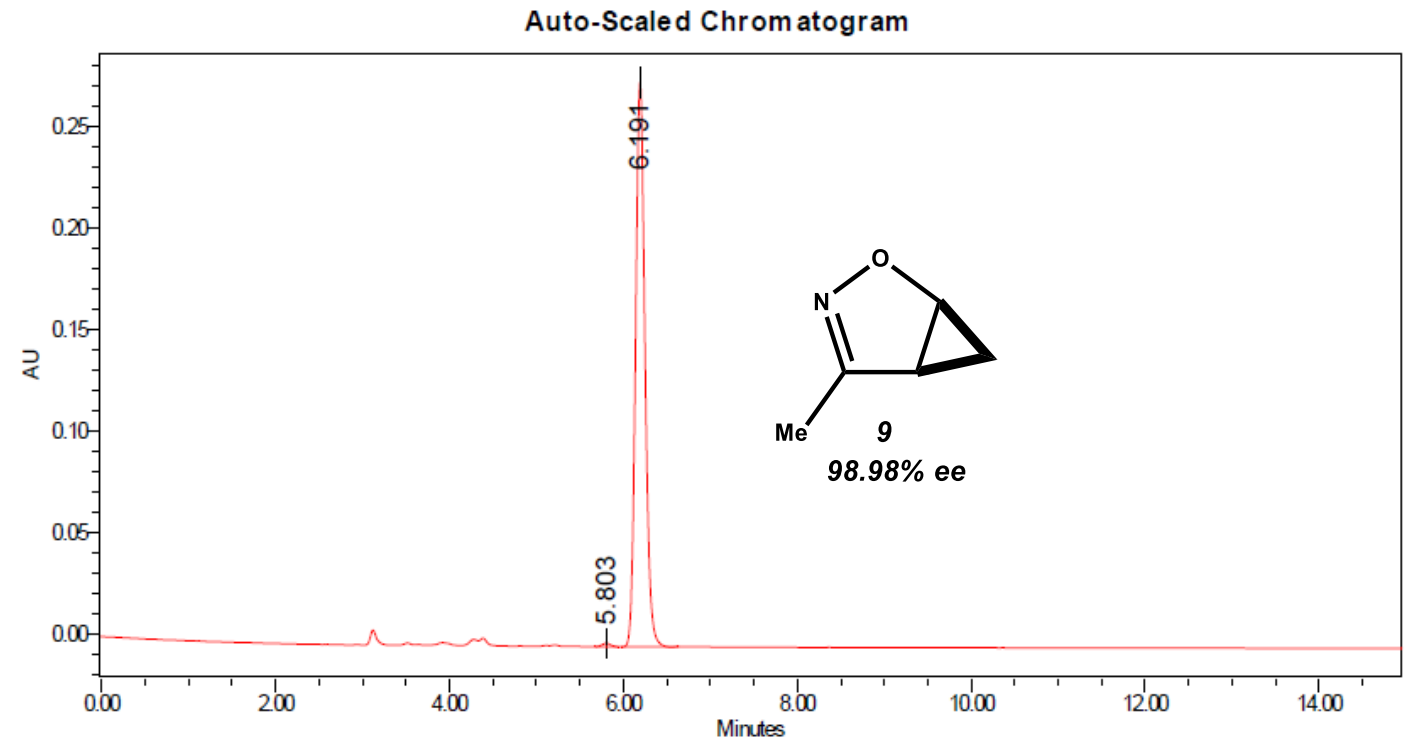

Processed Channel: DAD AU Ch 1 Sample

226, BW 8

\begin{tabular}{|l|c|r|r|r|r|}
\hline & Processed Channel & $\begin{array}{r}\text { Retention } \\
\text { Time (min) }\end{array}$ & Area & \% Area & Height \\
\hline 1 & DAD AU Ch 1 Sample 226, Bw8 & 5.803 & 10627 & 0.51 & 1650 \\
\hline 2 & DAD AU Ch 1 Sample 226, Bw8 & 6.191 & 2126330 & 99.49 & 278396 \\
\hline
\end{tabular}

Auto-Scaled Chrom atogram

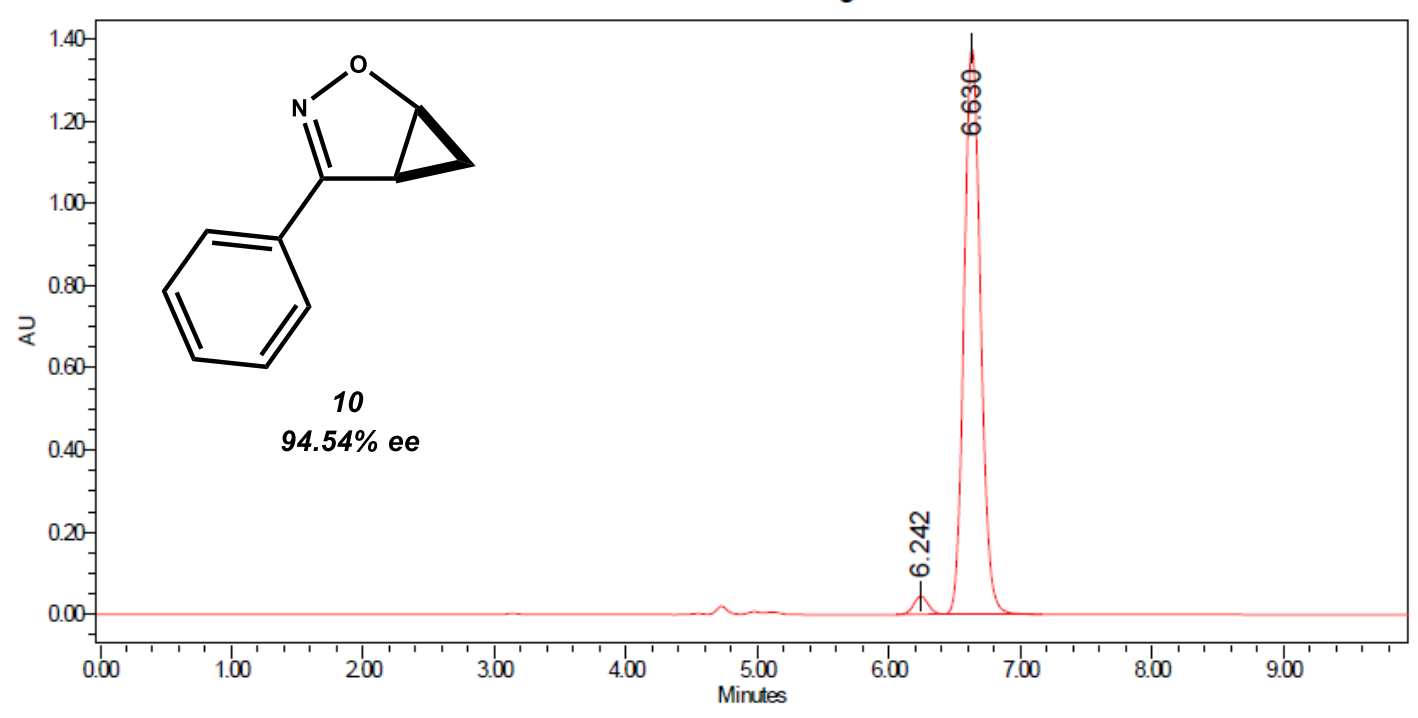

Processed Channel: DAD AU Ch 1 Sample 226, Bw 8

\begin{tabular}{|r|c|r|r|r|r|}
\hline & Processed Channel & $\begin{array}{c}\text { Retertion } \\
\text { Time(min) }\end{array}$ & Area & \% Area & Height \\
\hline 1 & DAD AU Ch 1 Sample 226, Bw8 & 6.242 & 336730 & 273 & 43726 \\
\hline 2 & DAD AU Ch 1 Sample 226, Bw8 & 6.630 & 11968532 & 97.27 & 1375626 \\
\hline
\end{tabular}


Auto-Scaled Chrom atogram

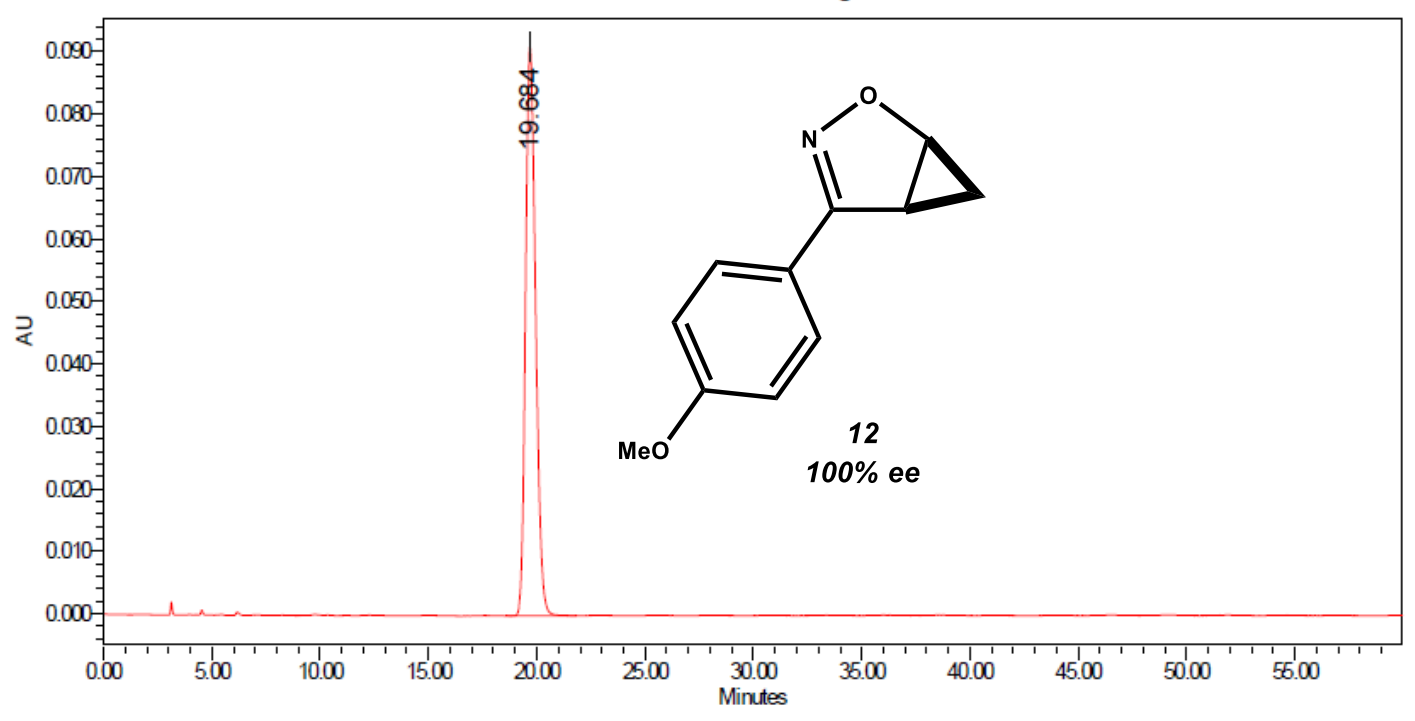

Processed Channel: DAD AU Ch 1 Sample

226, BW 8

\begin{tabular}{|c|c|r|c|c|c|}
\hline & Processed Channel & $\begin{array}{c}\text { Retention } \\
\text { Time (min) }\end{array}$ & Area & \% Area & Height \\
\hline 1 & DAD AU Ch 1 Sample 226, Bw8 & 19.684 & 2956229 & 100.00 & 91058 \\
\hline
\end{tabular}




\section{${ }^{1}$ H NMR Spectra}




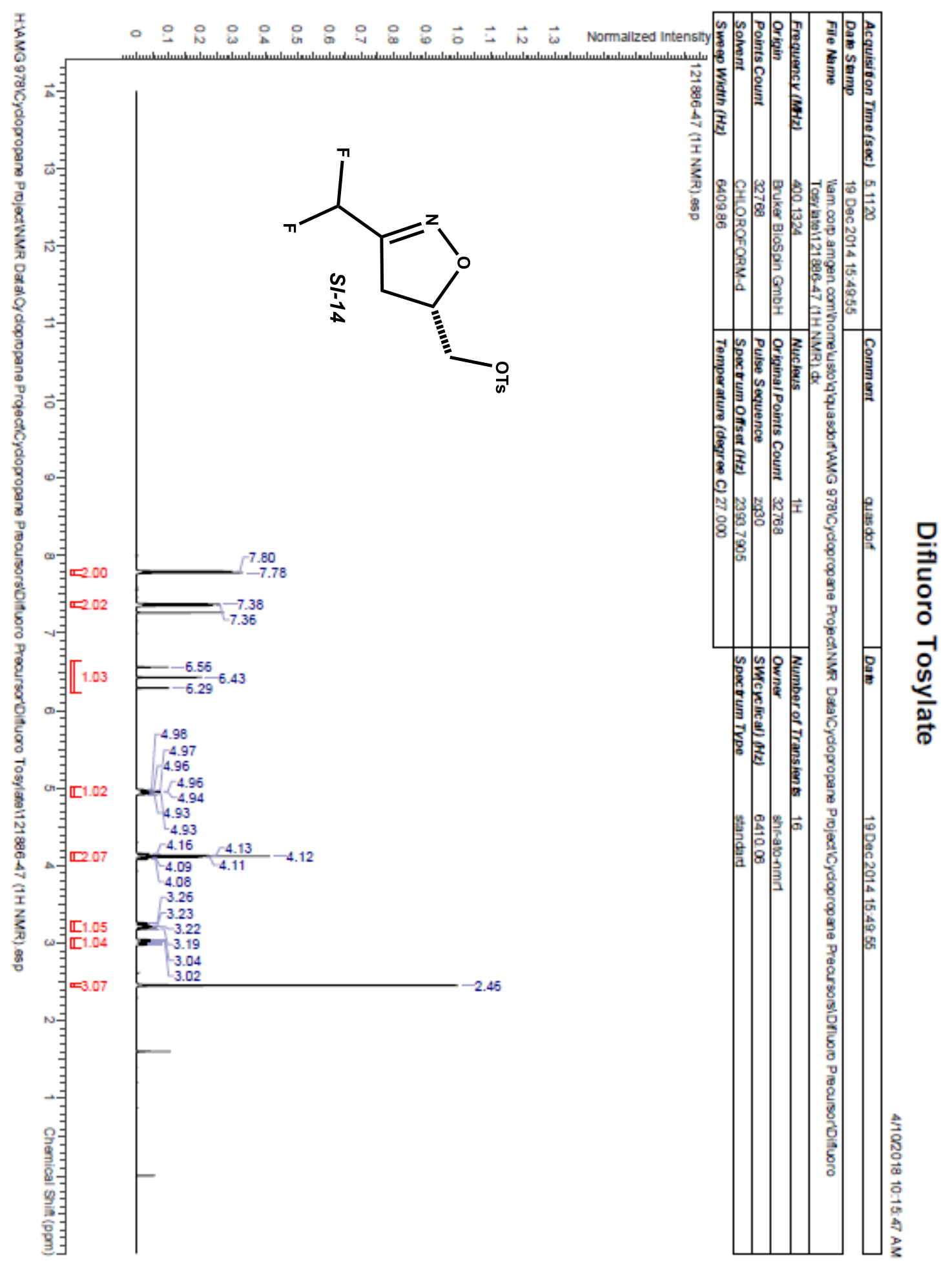




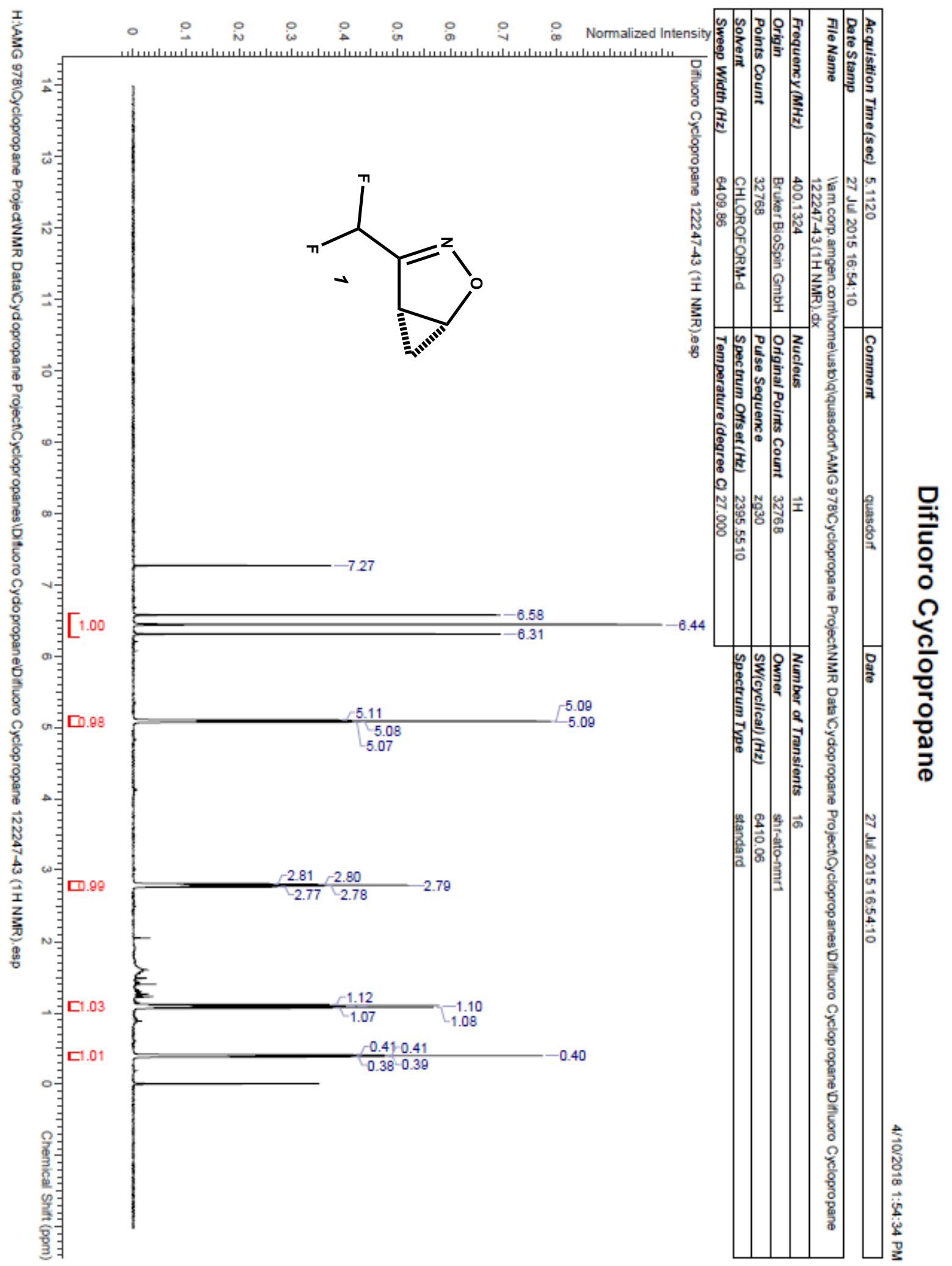




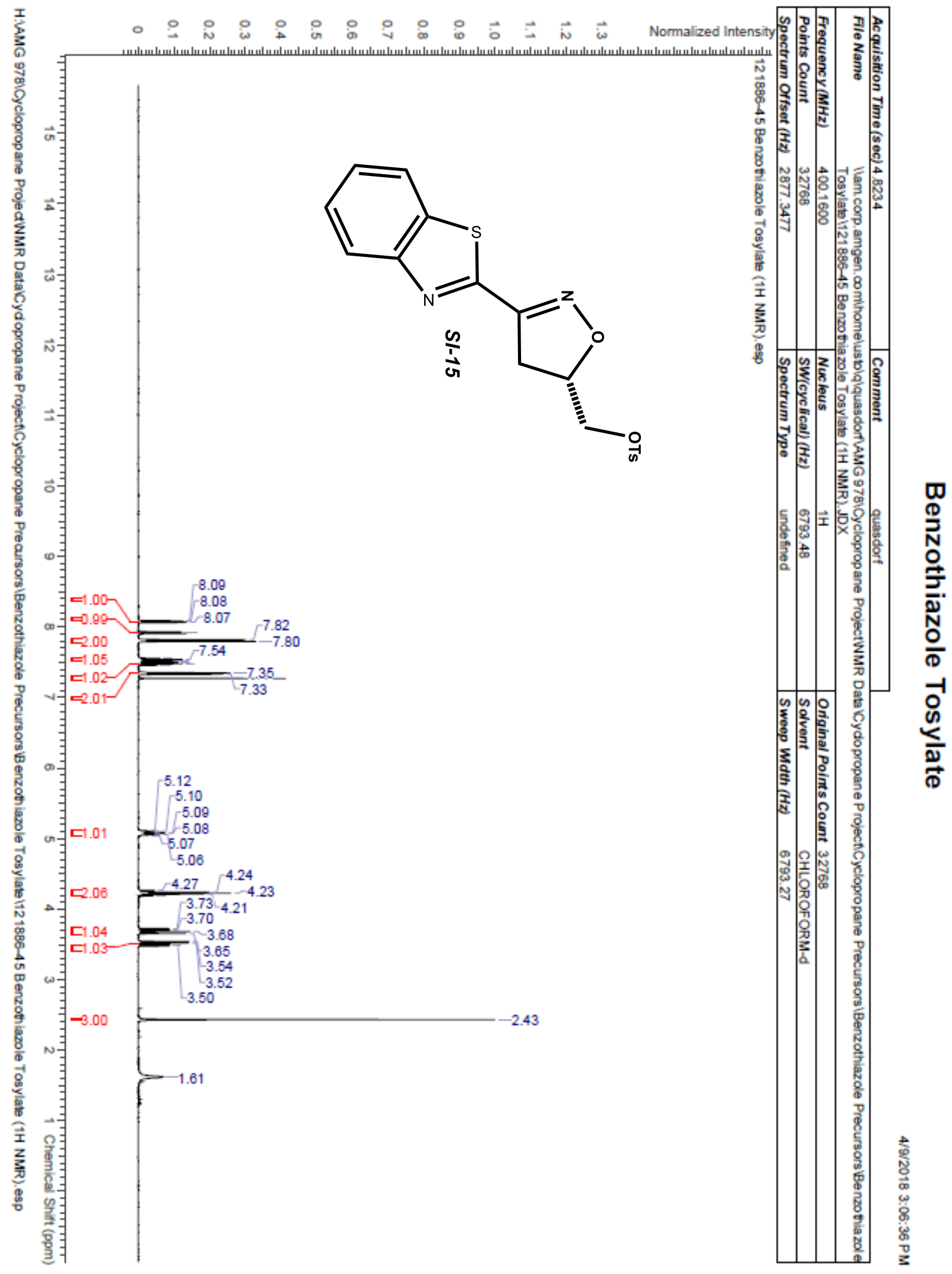




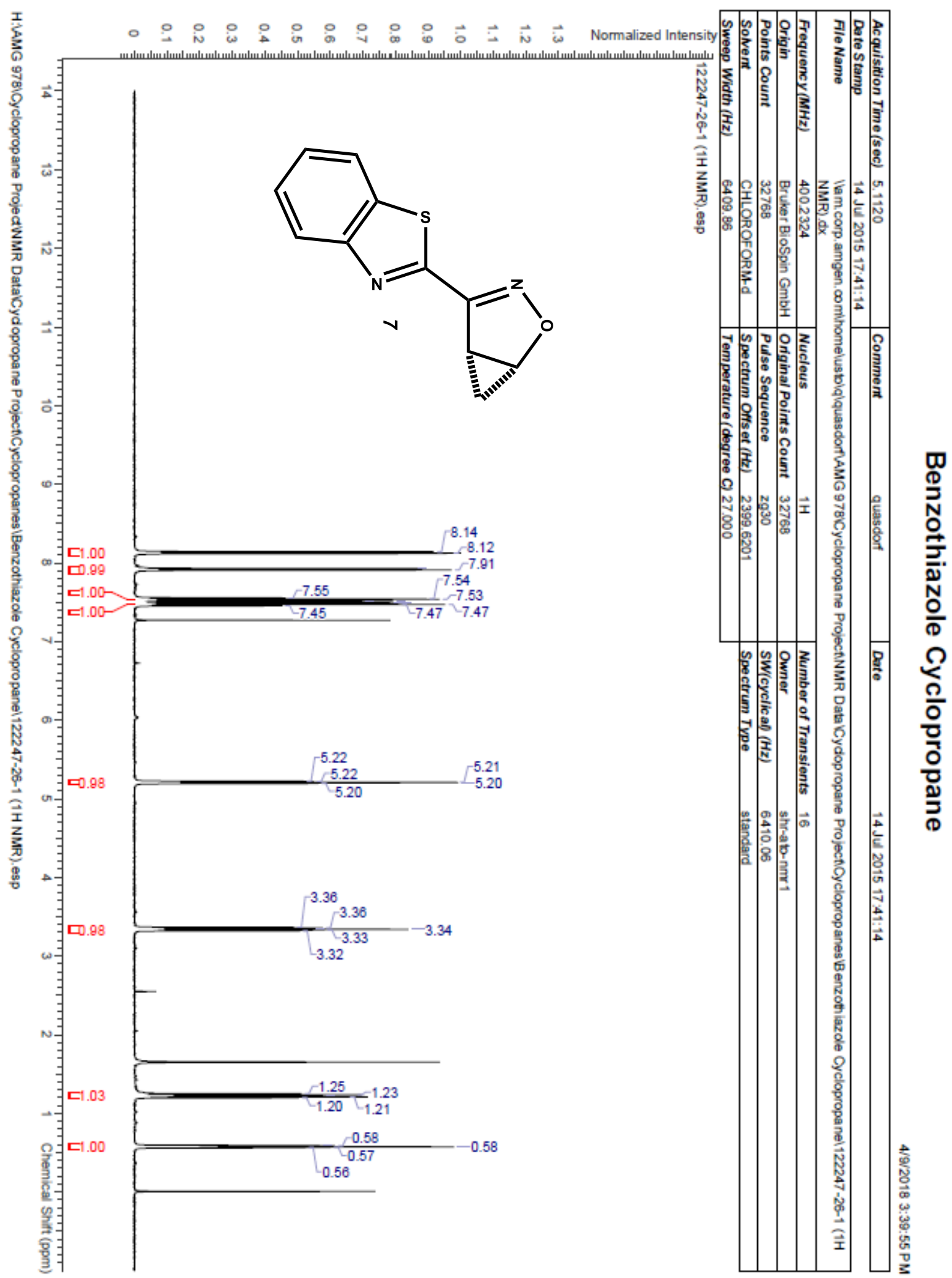




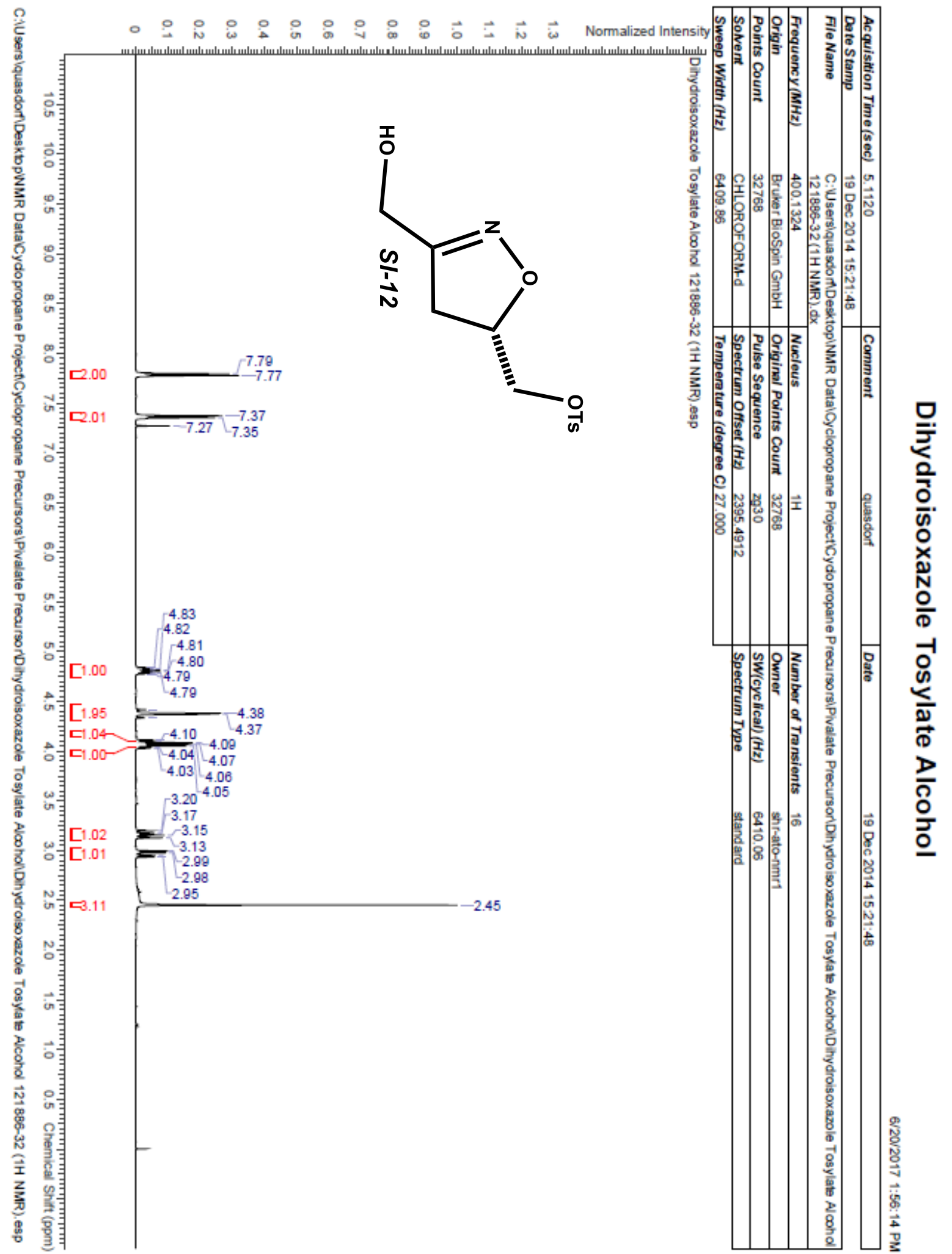




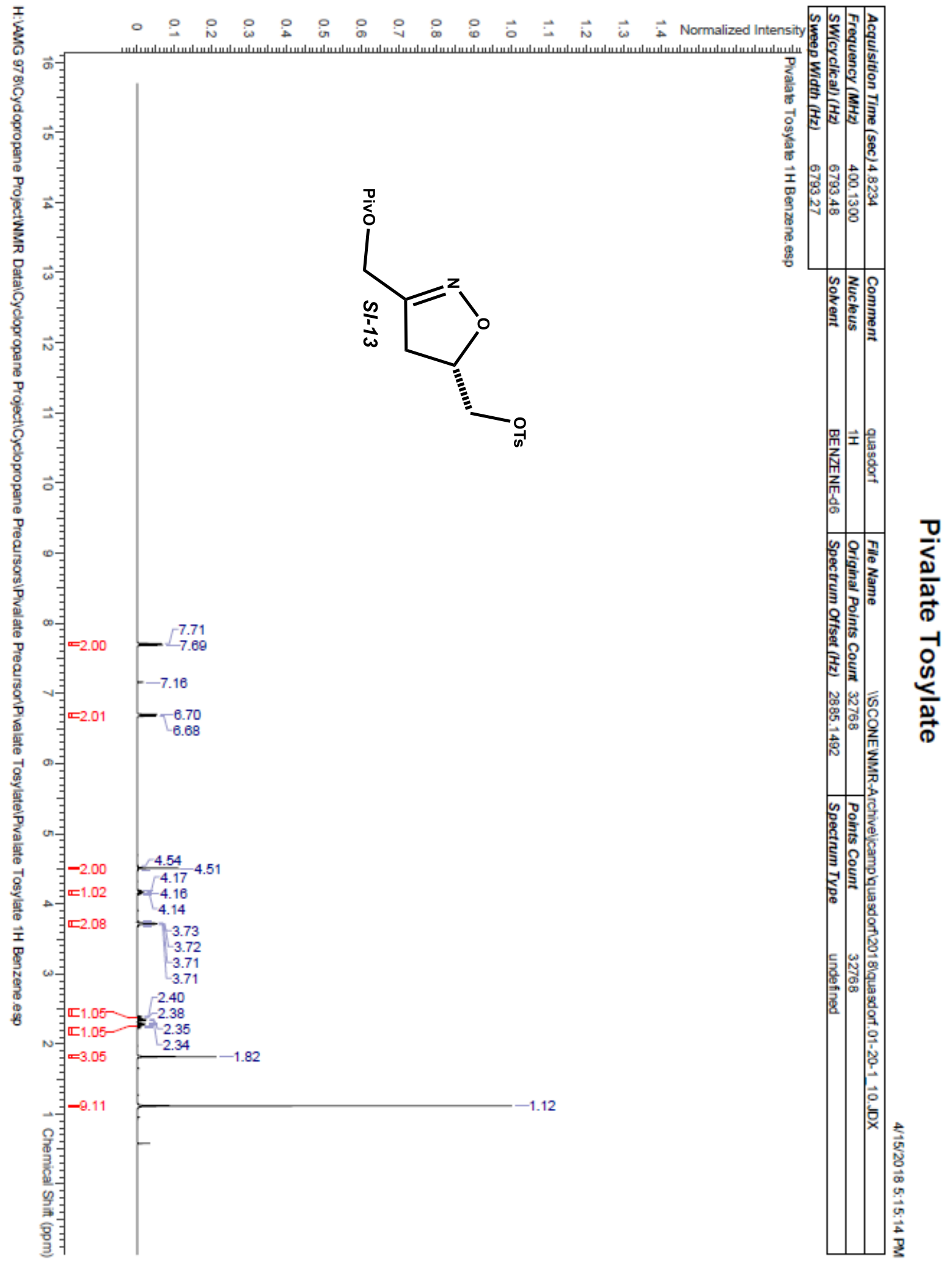




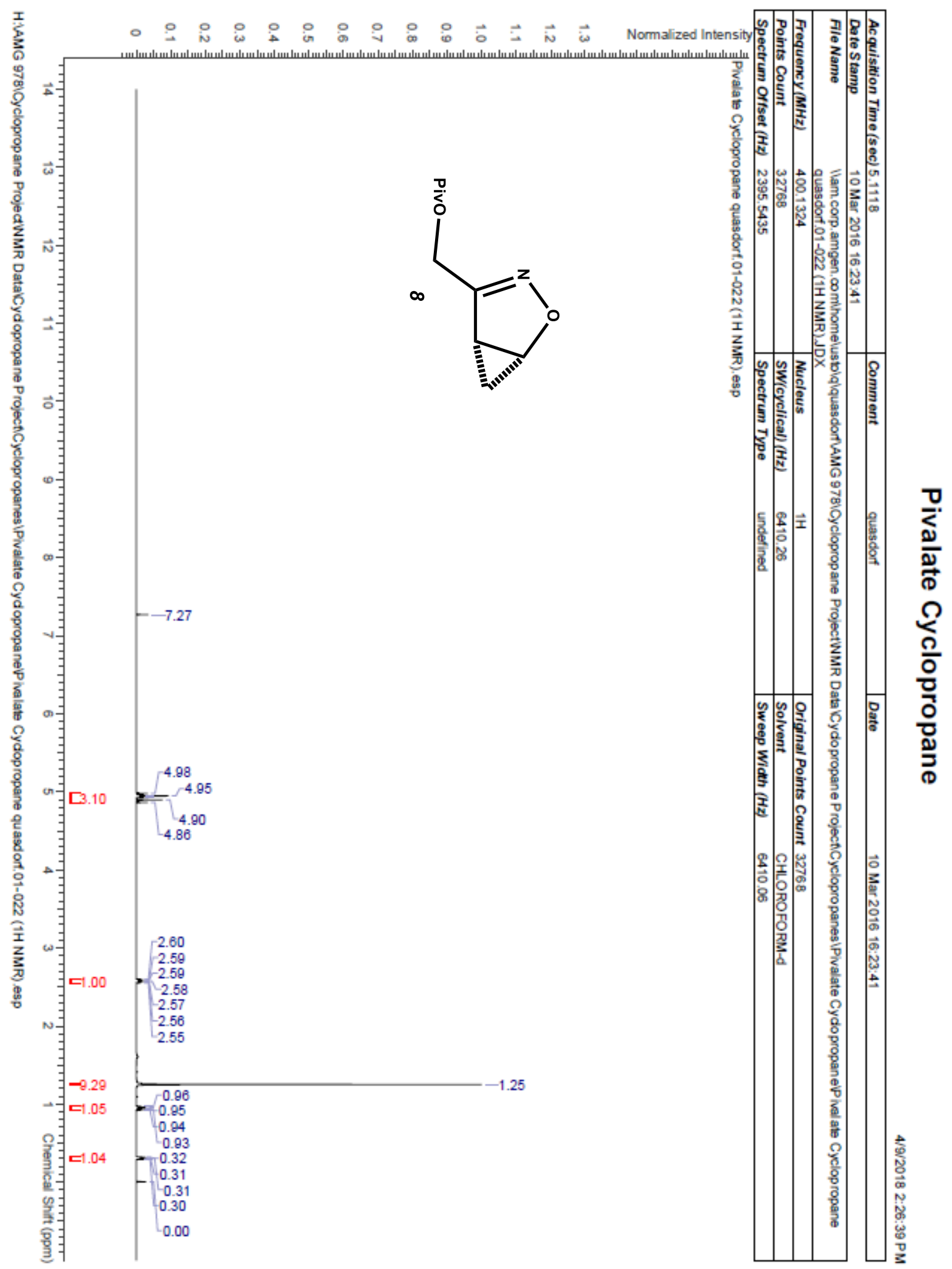




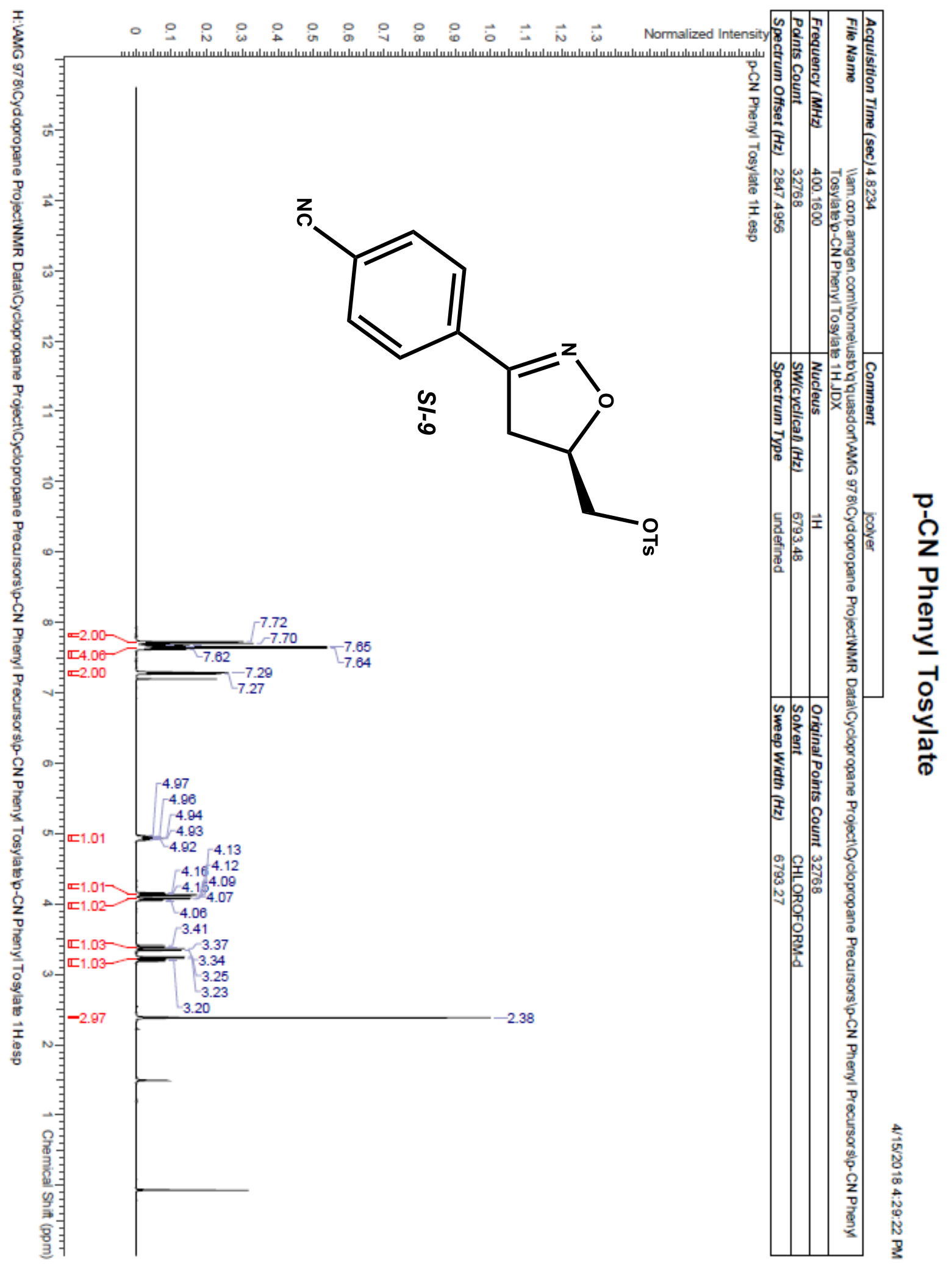




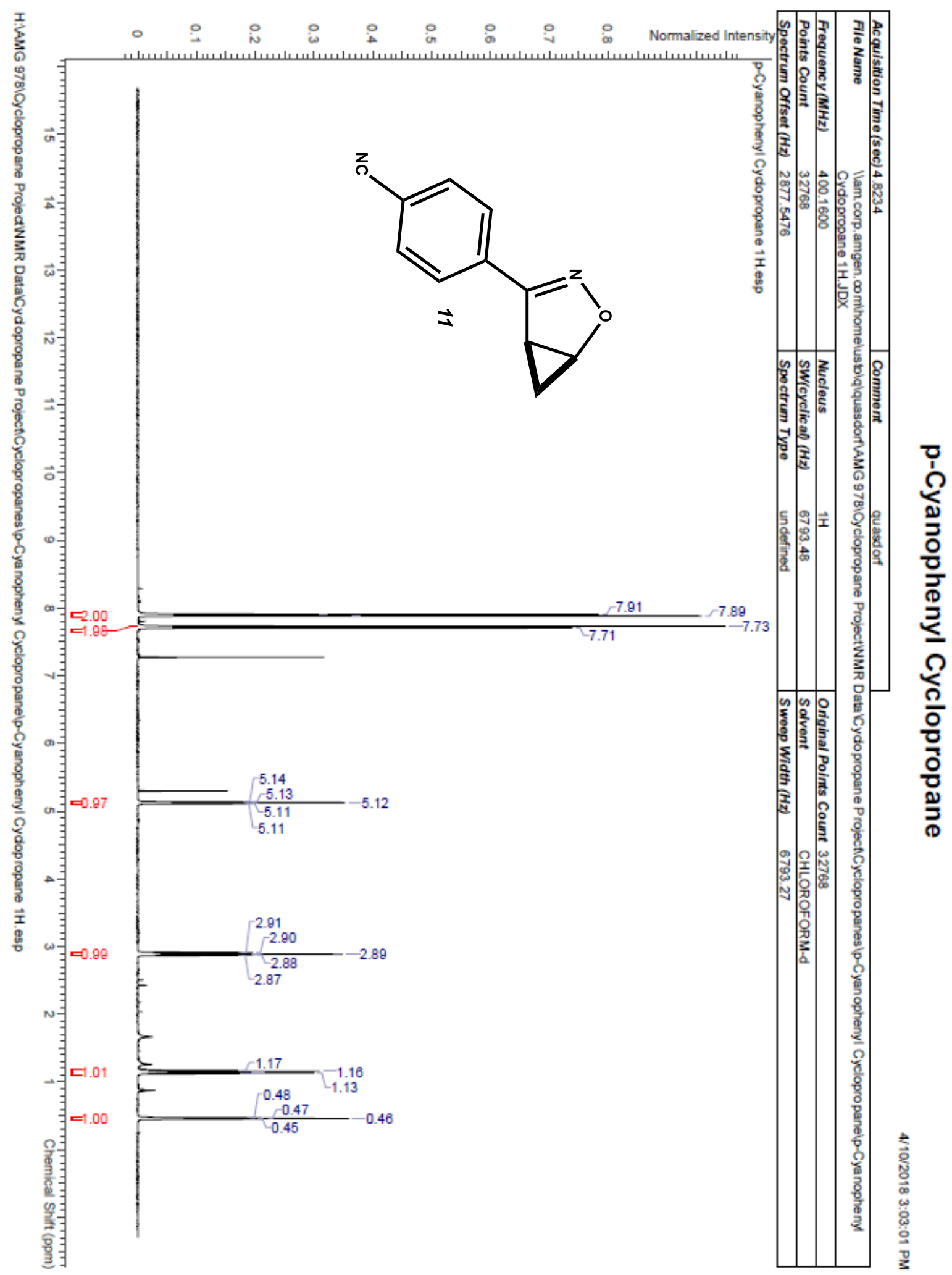




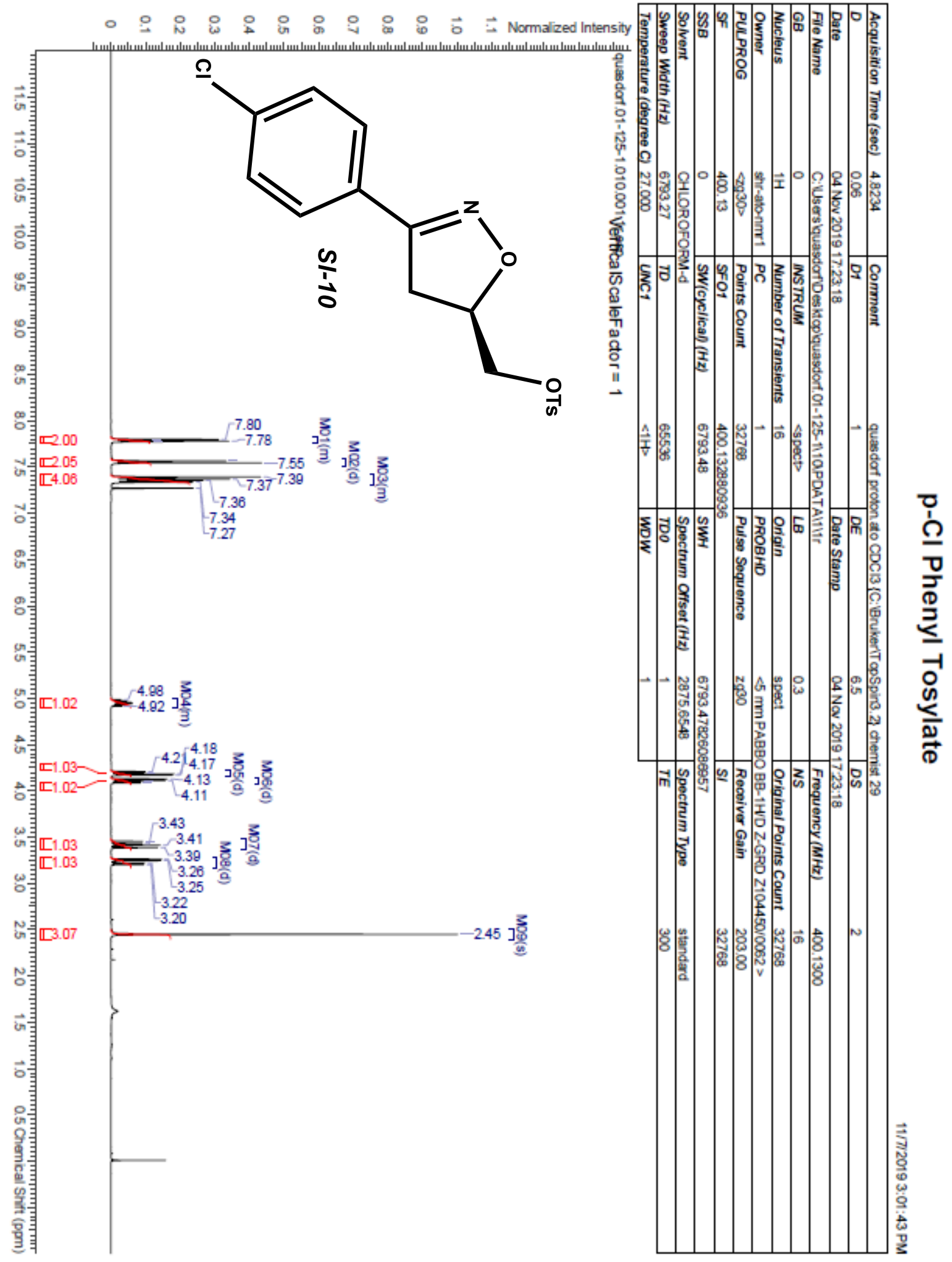




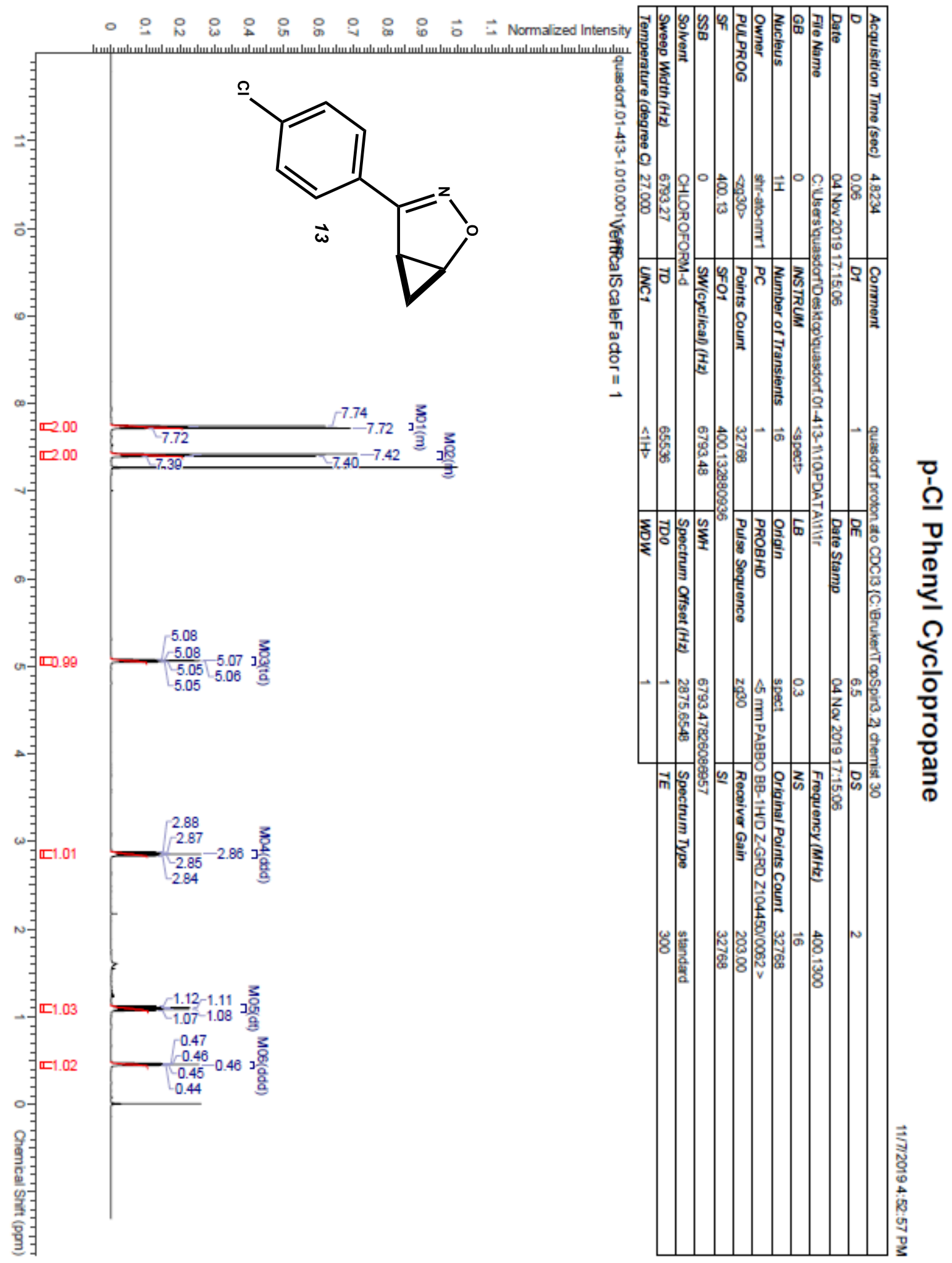




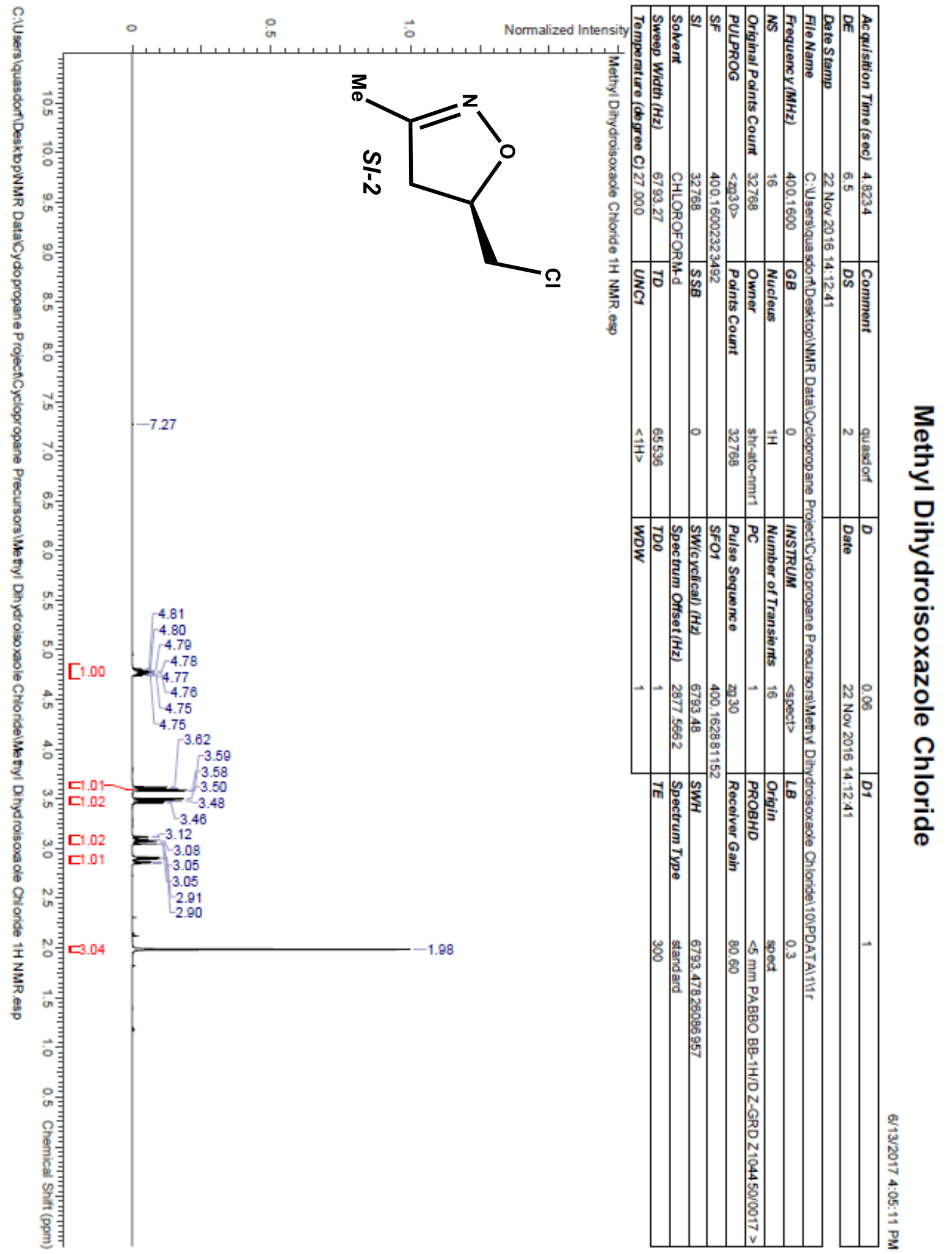




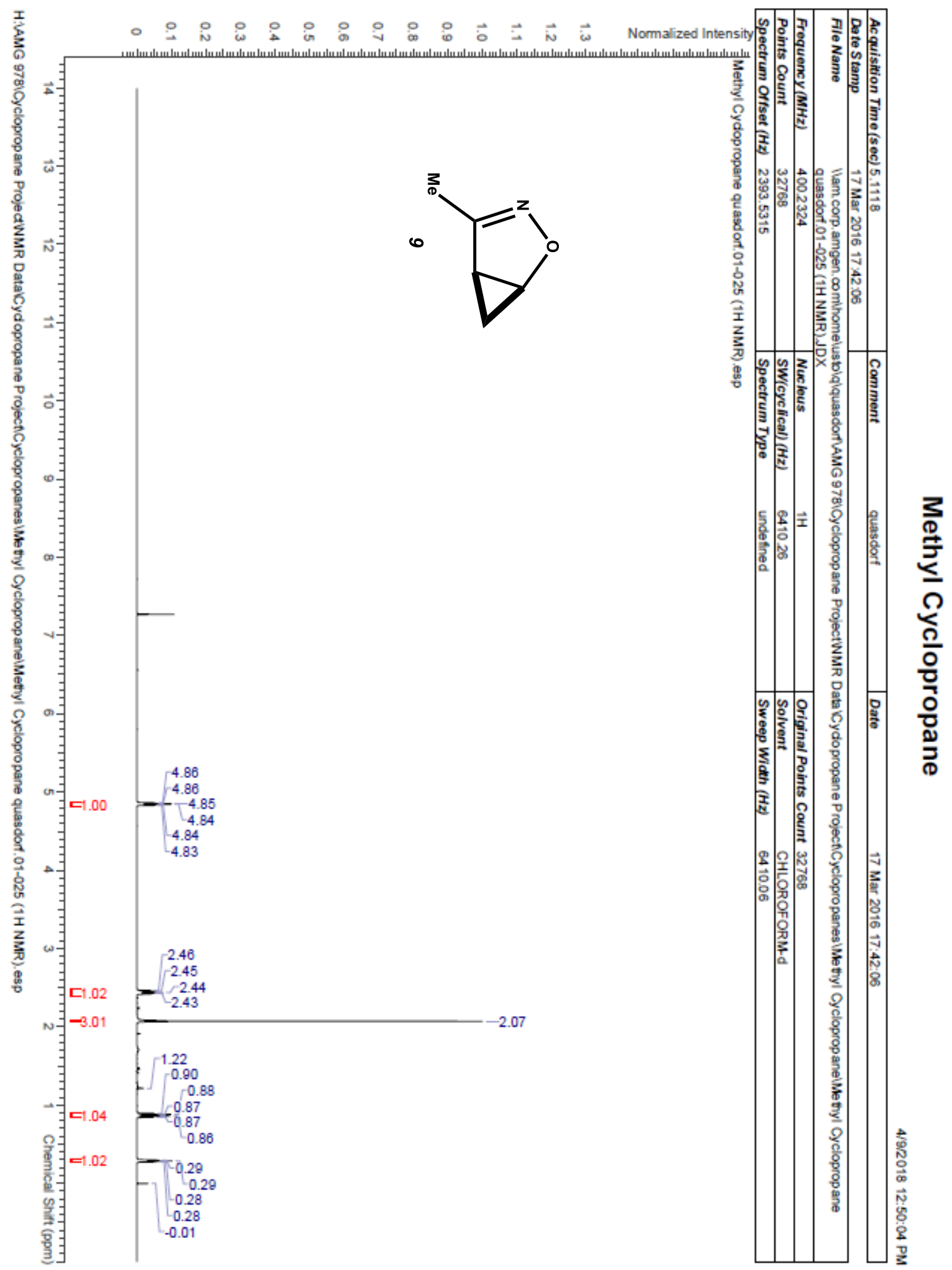




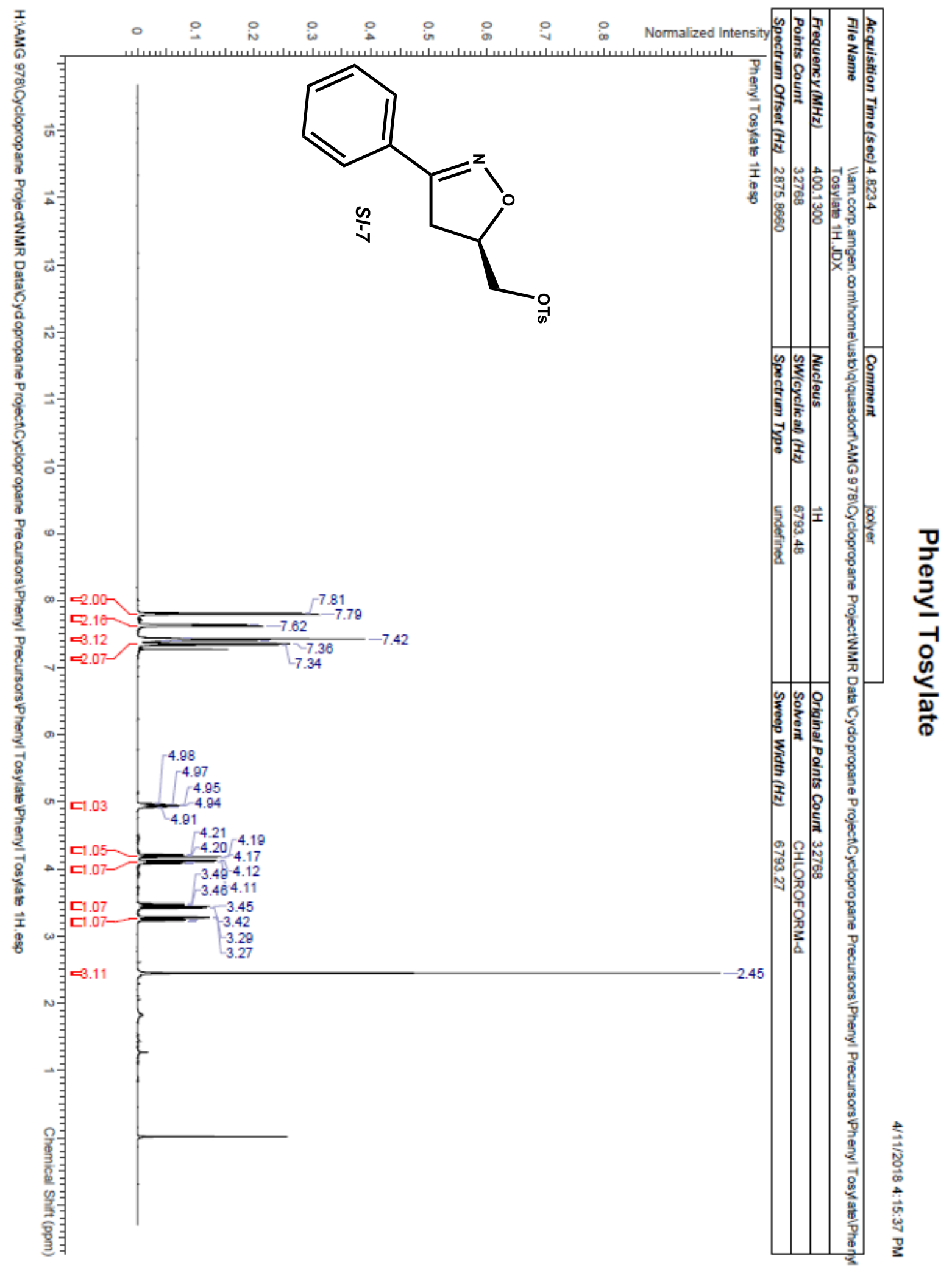




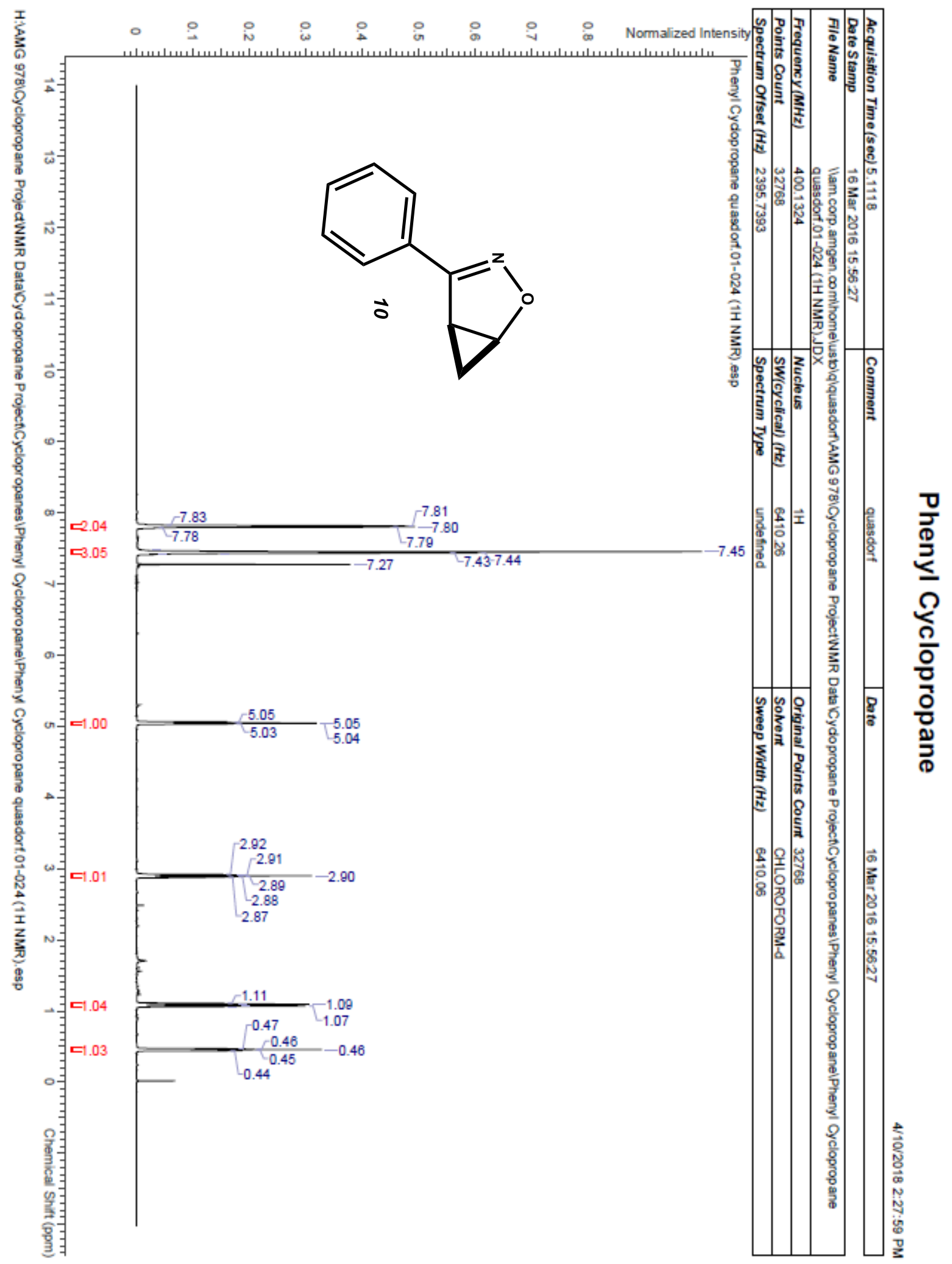




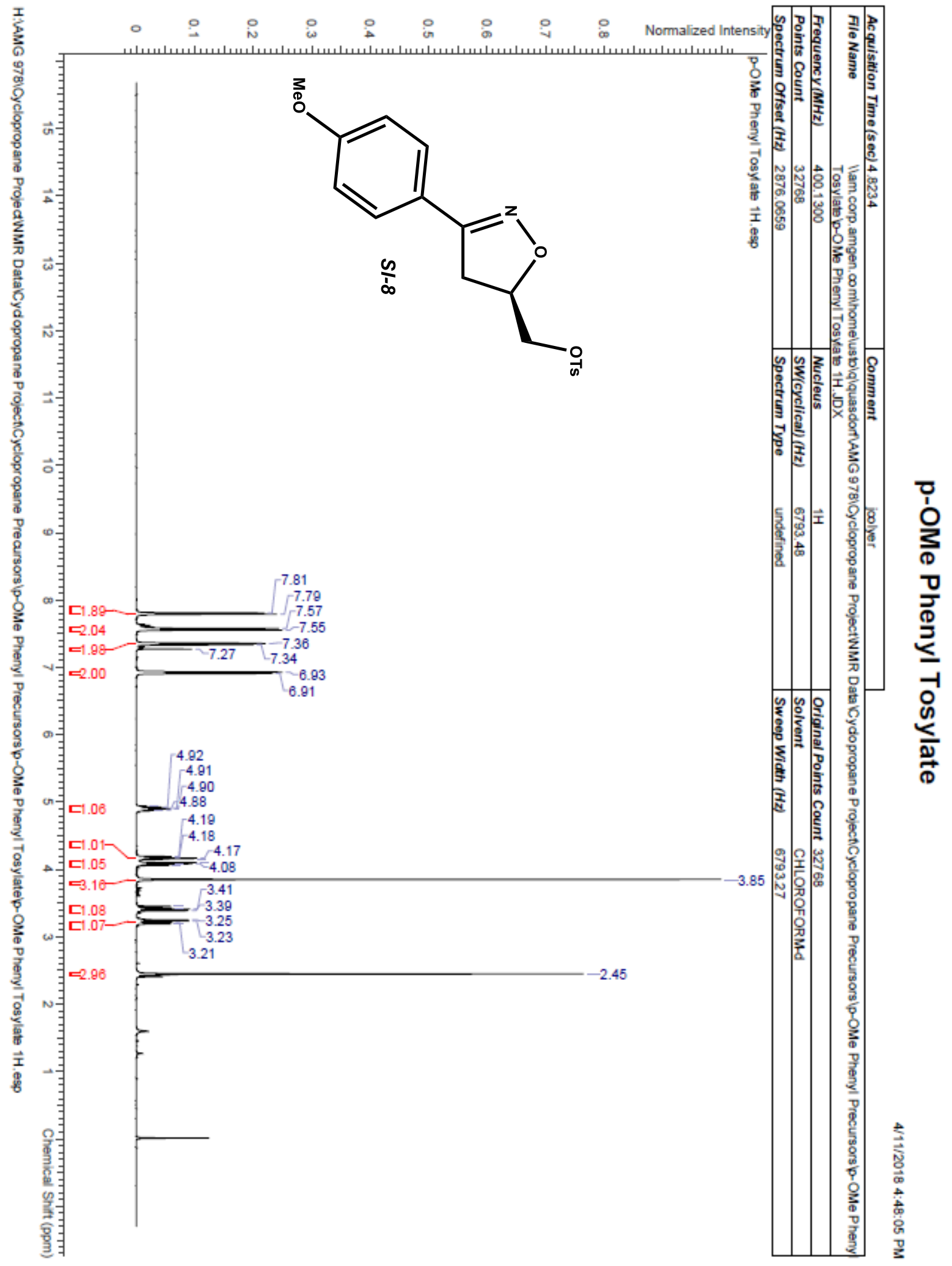




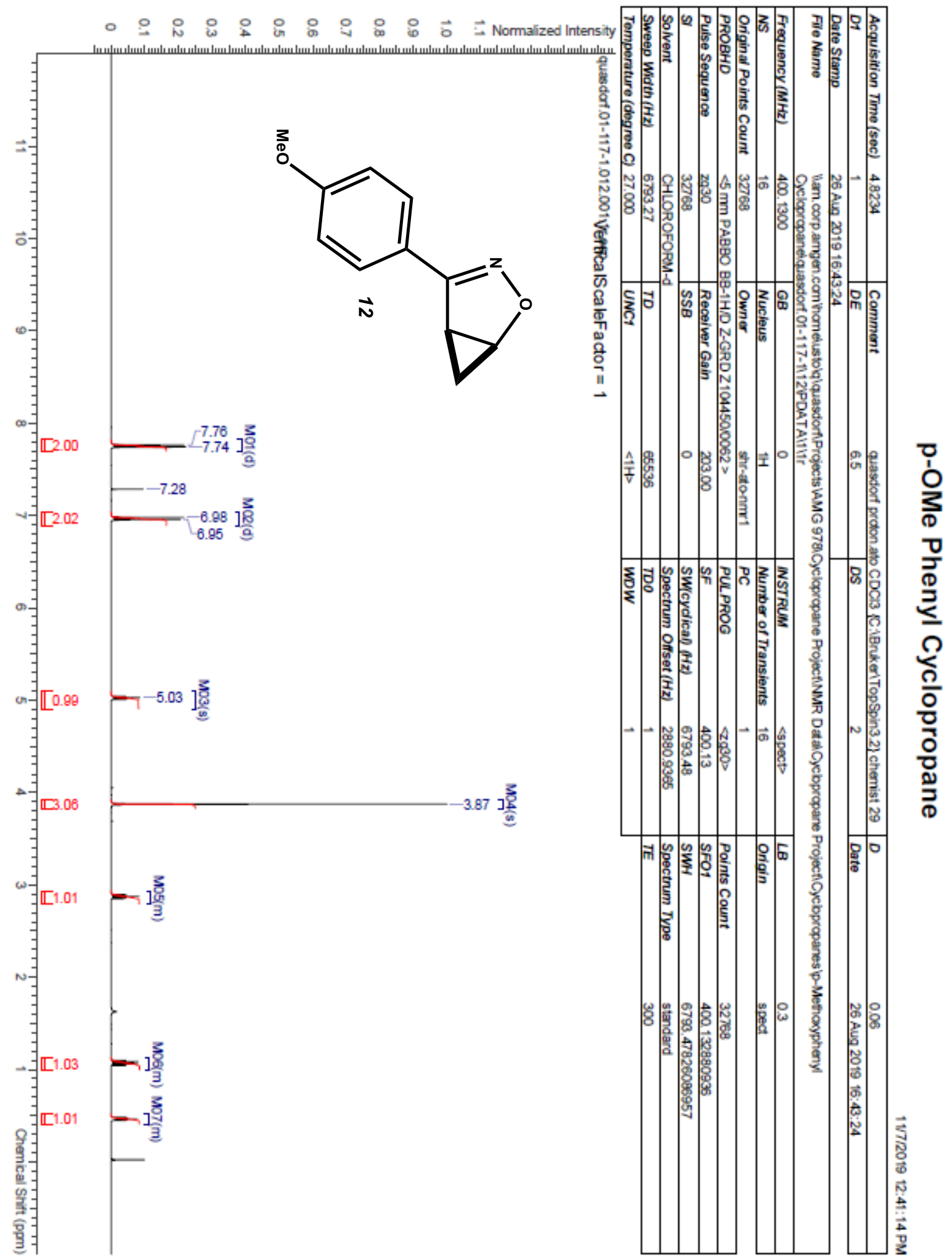




\section{${ }^{13}$ C NMR Spectra}




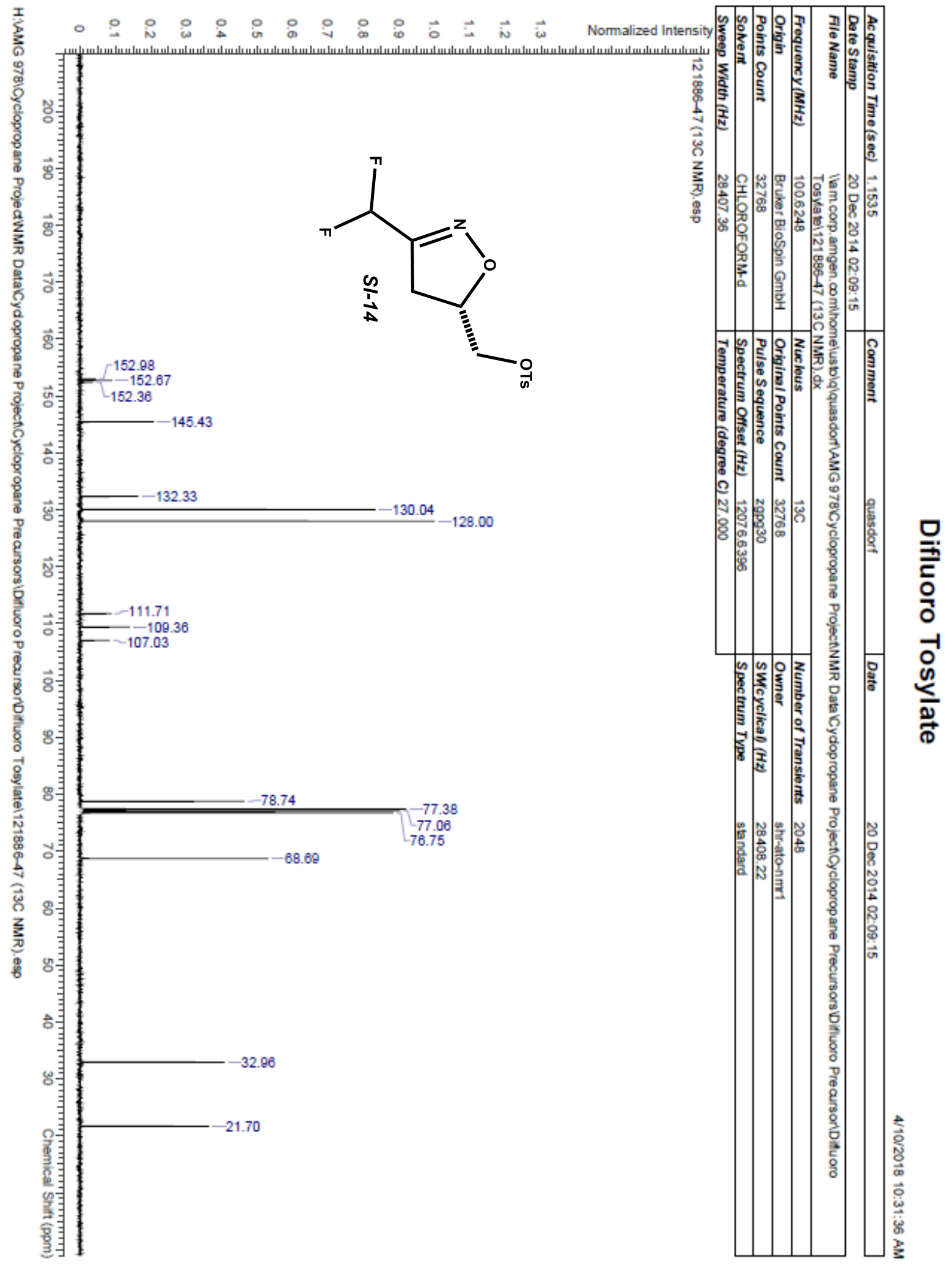




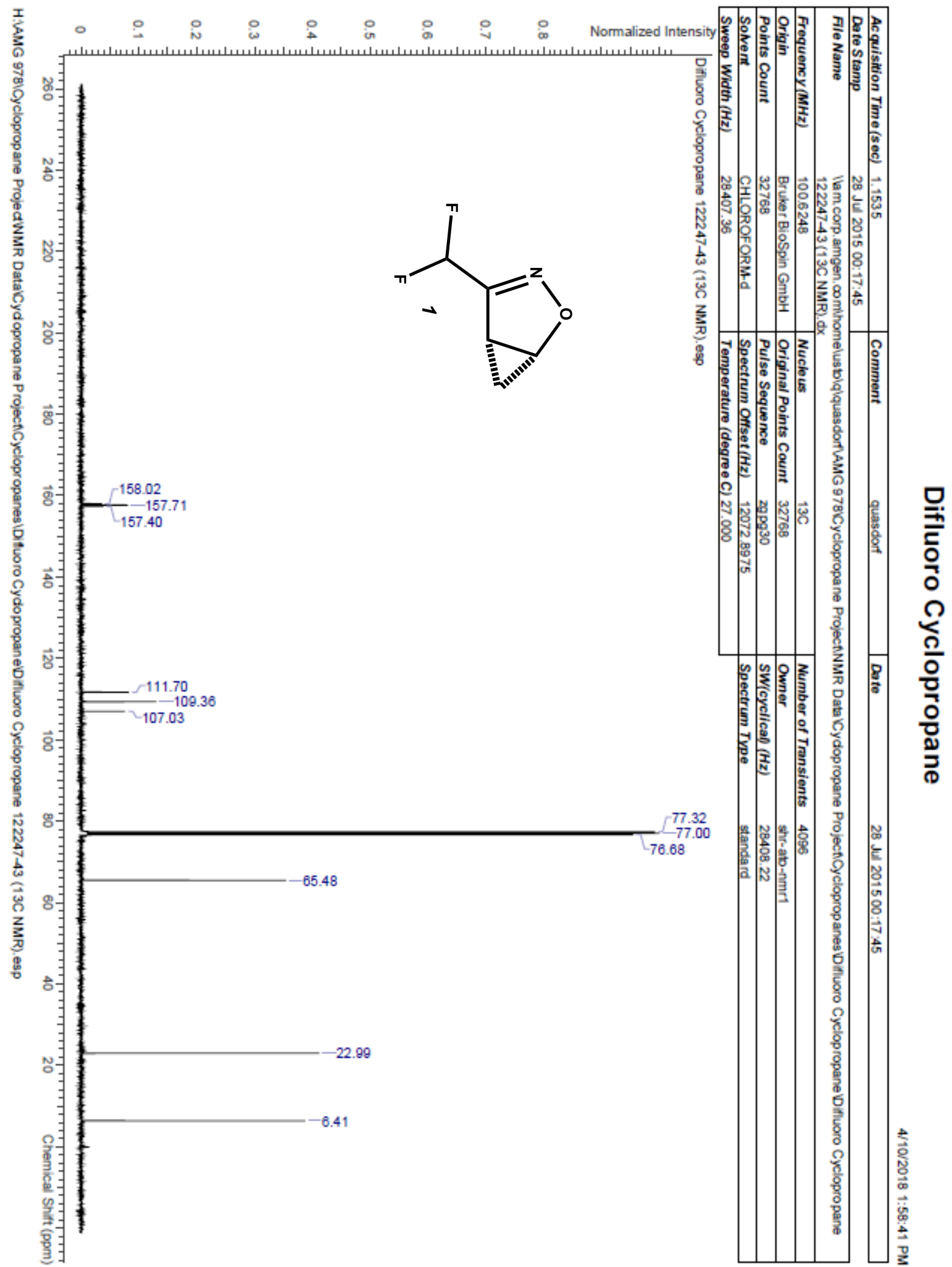




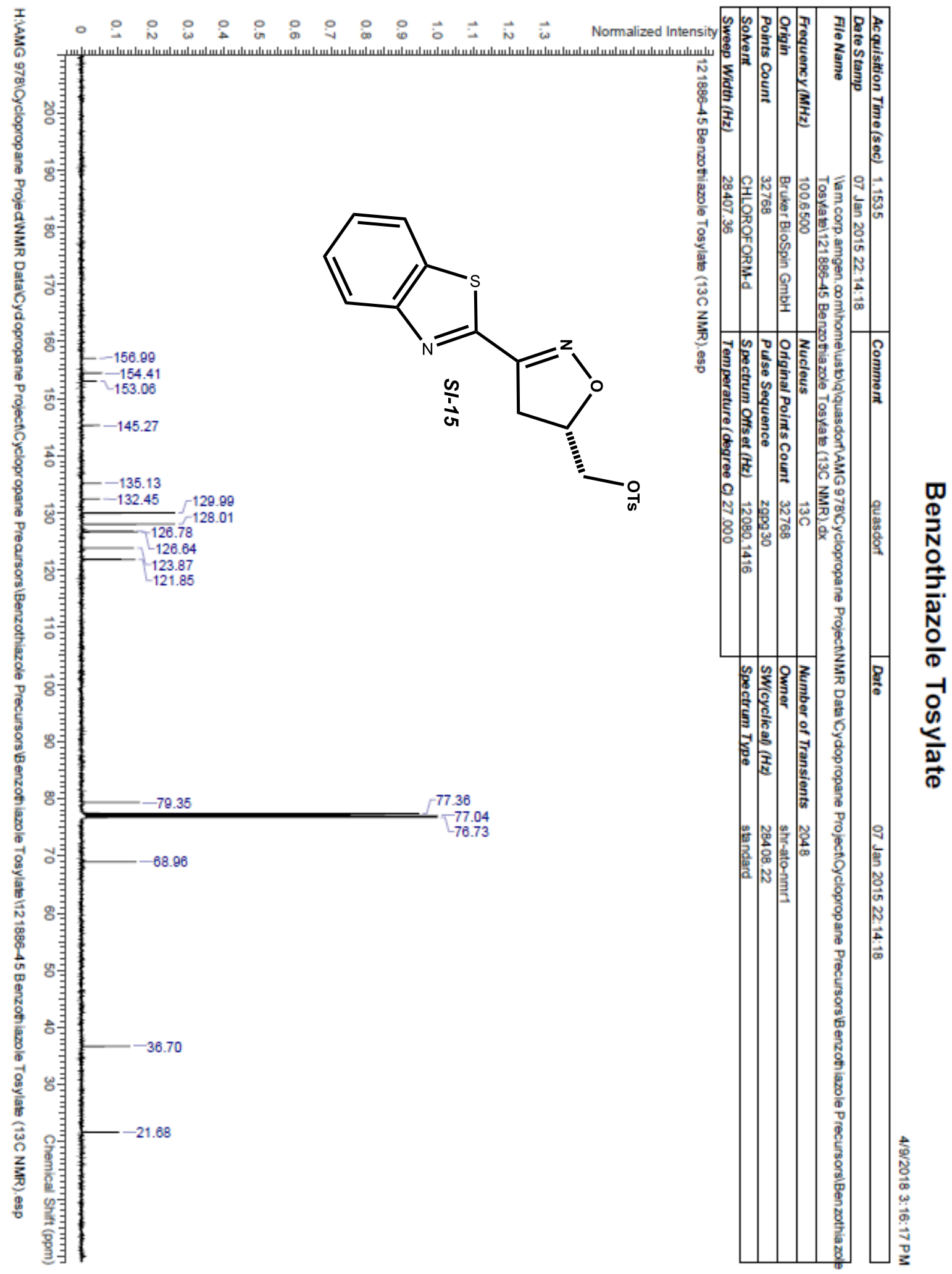




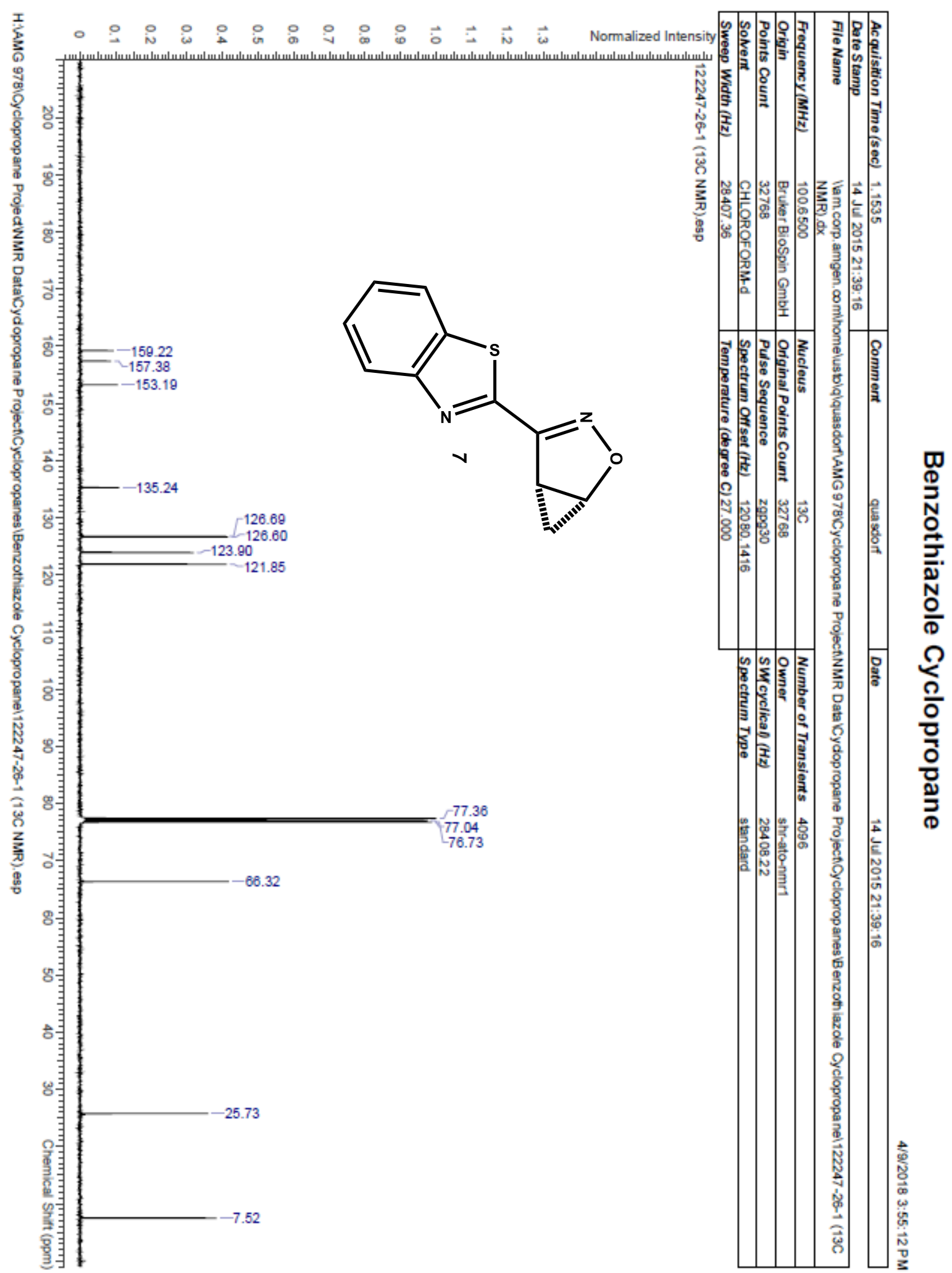




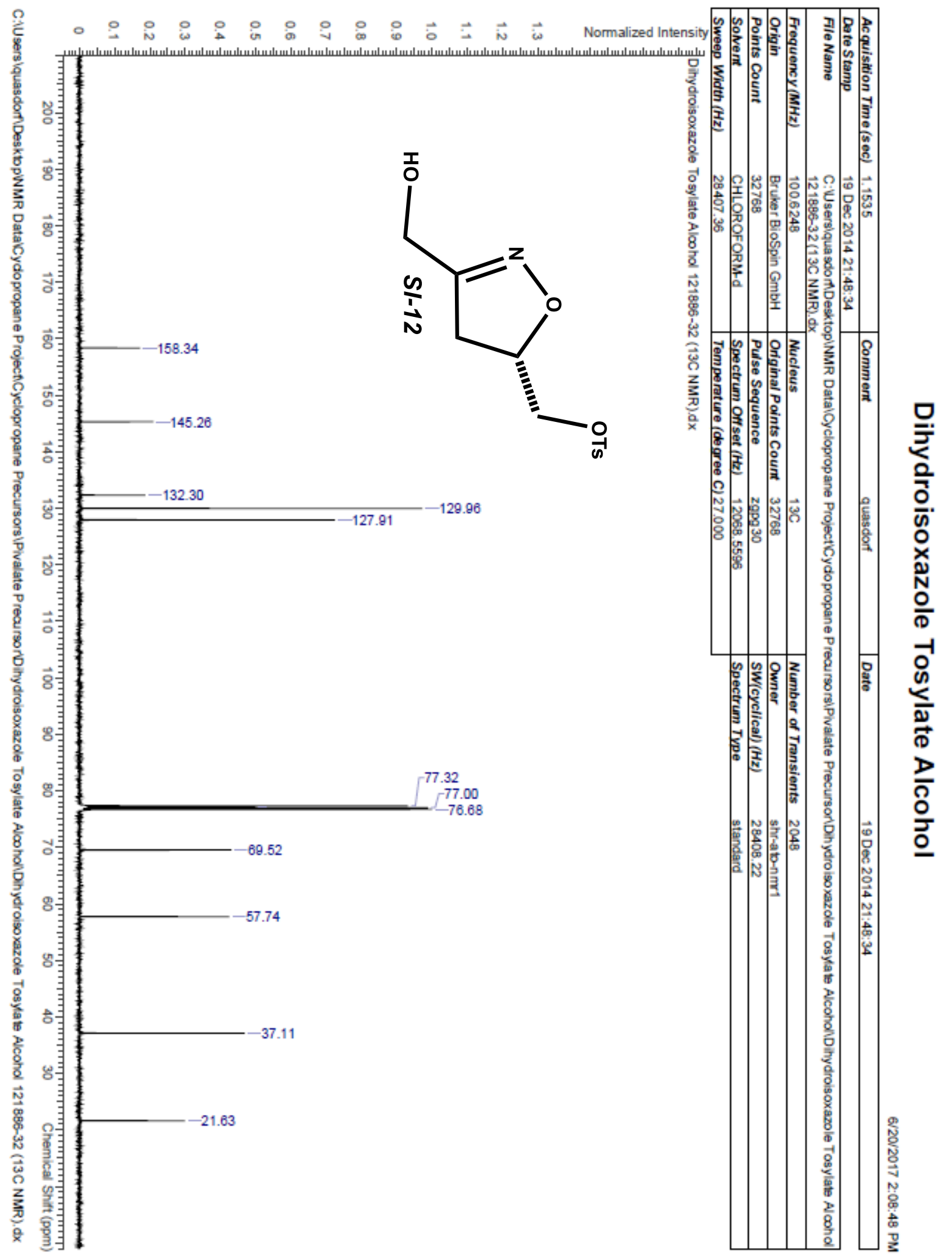




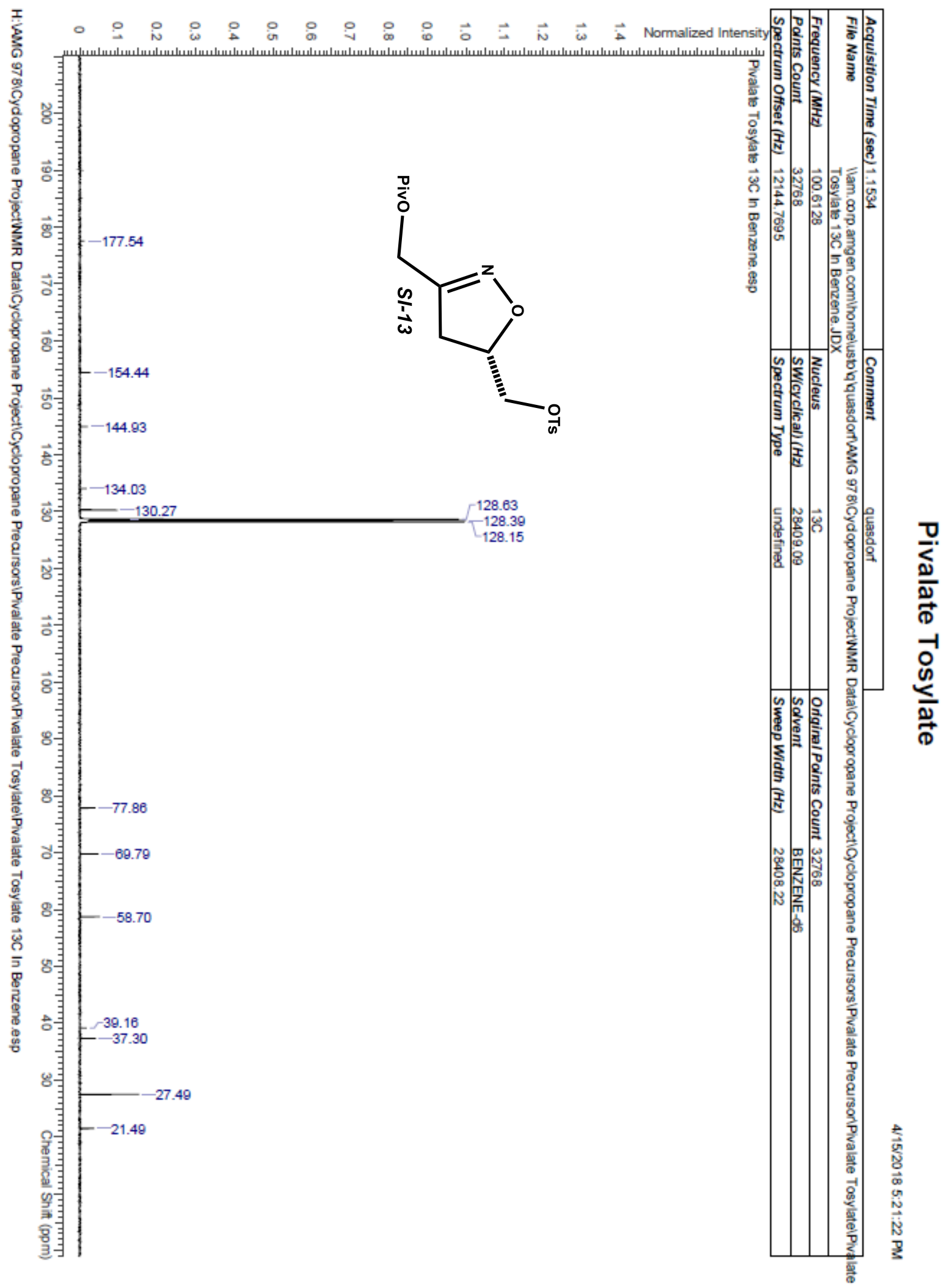




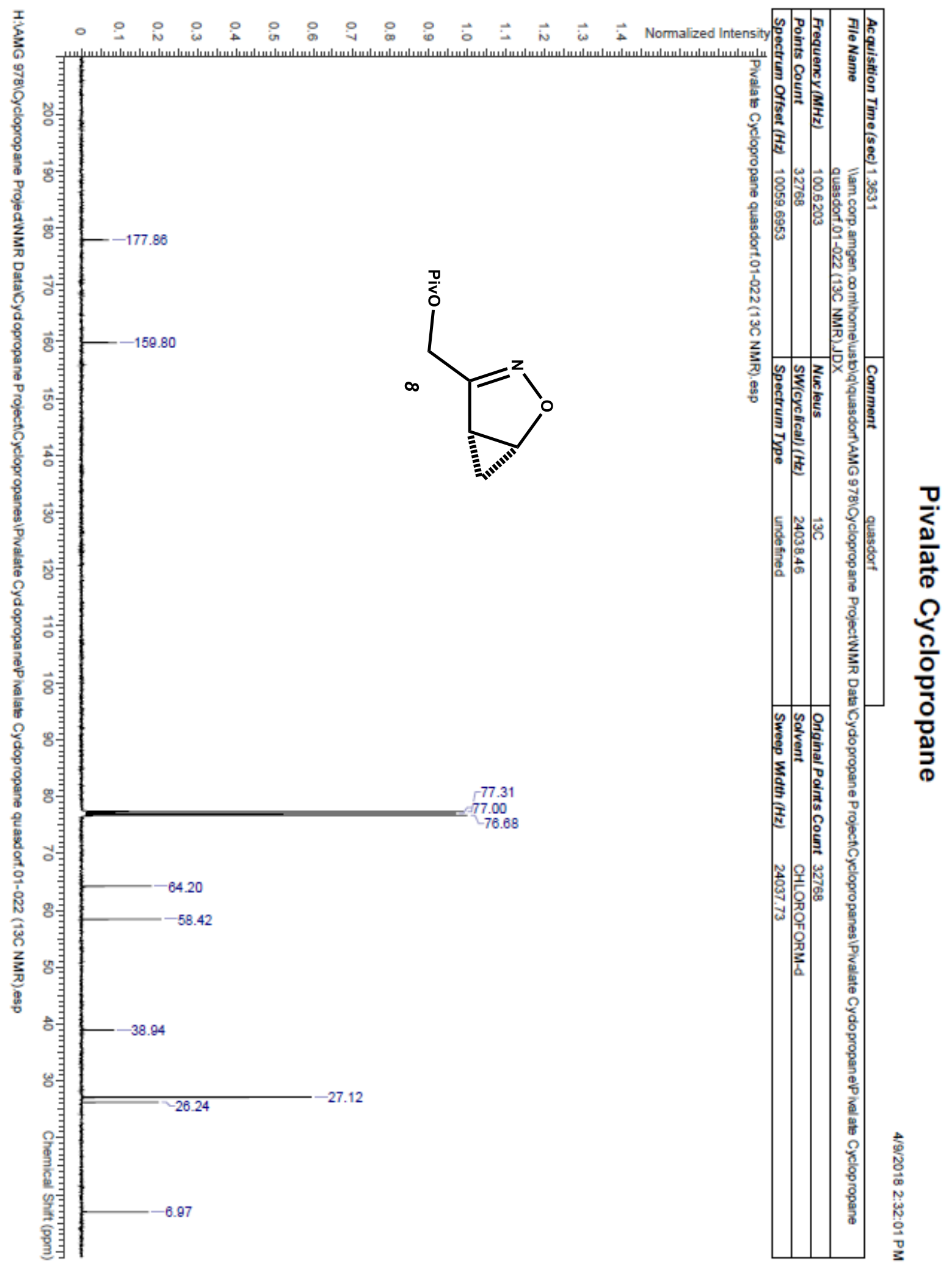




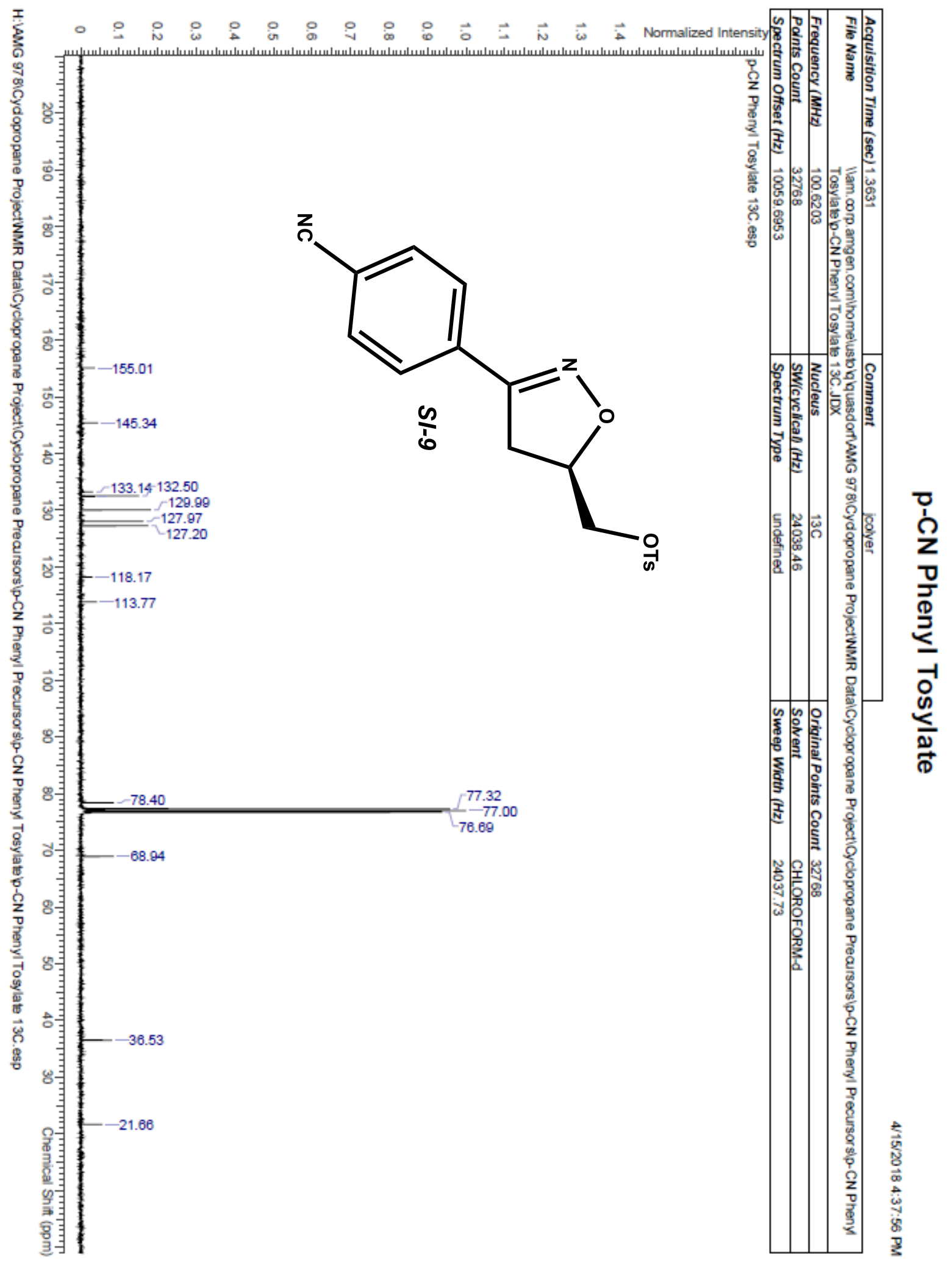




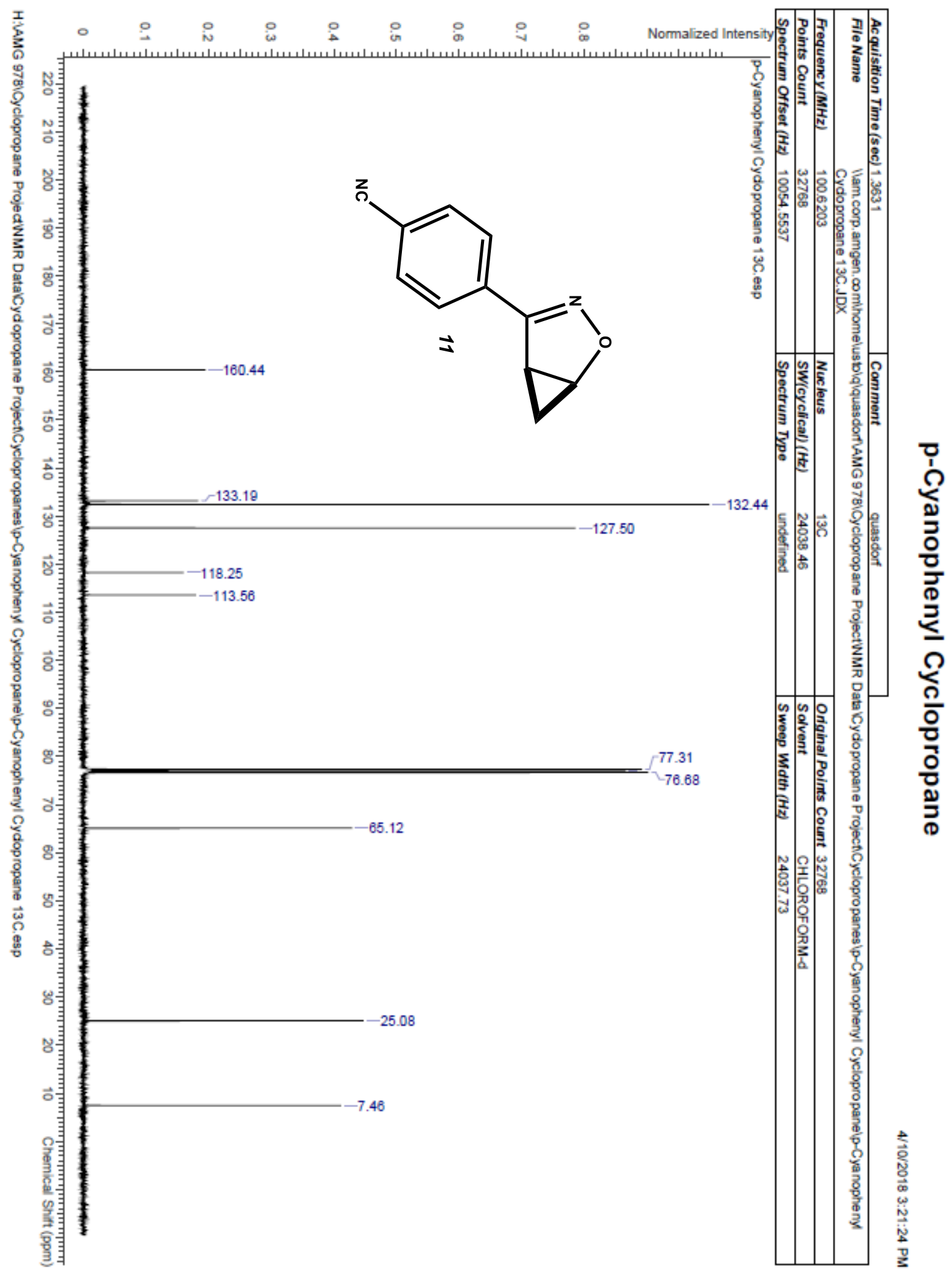




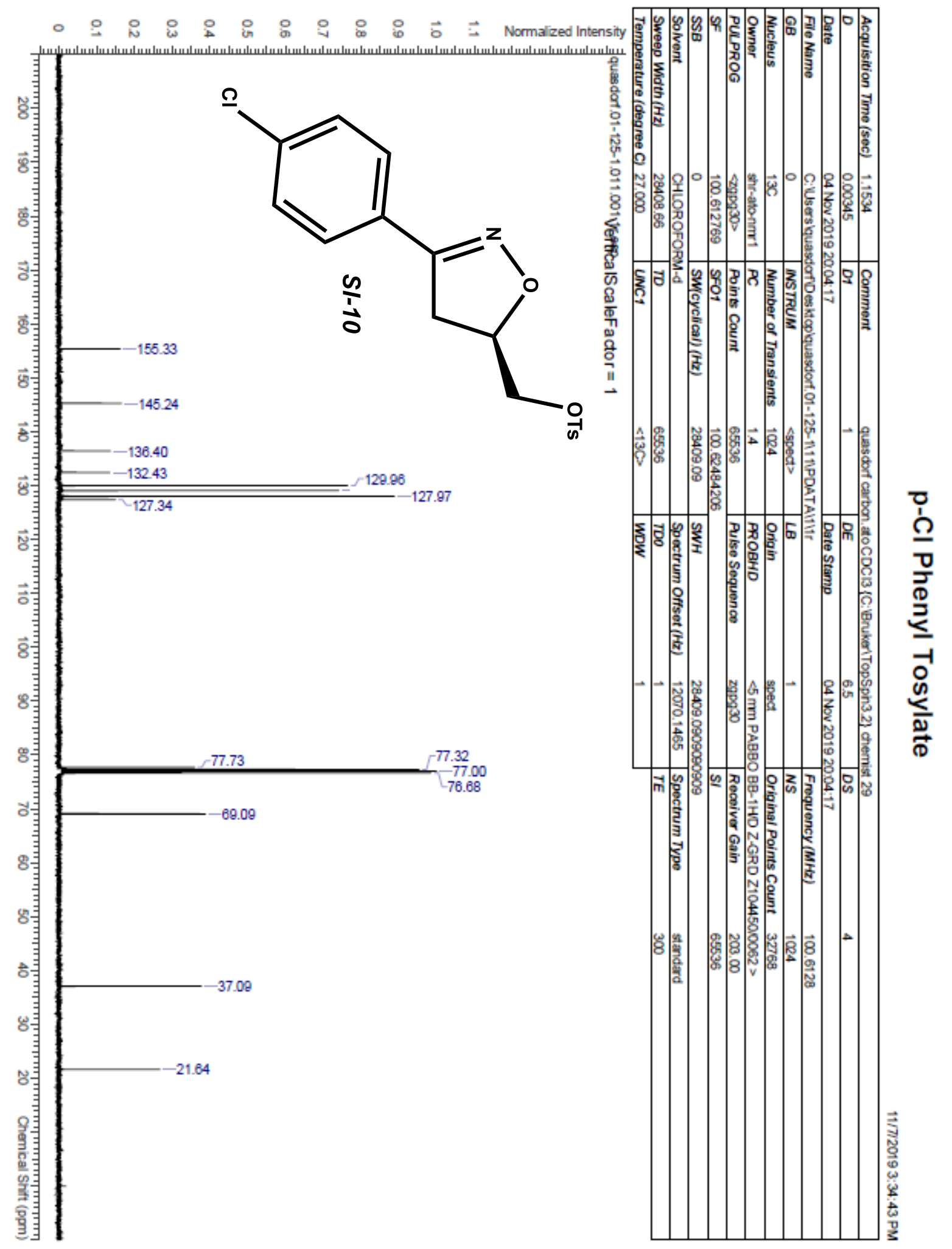




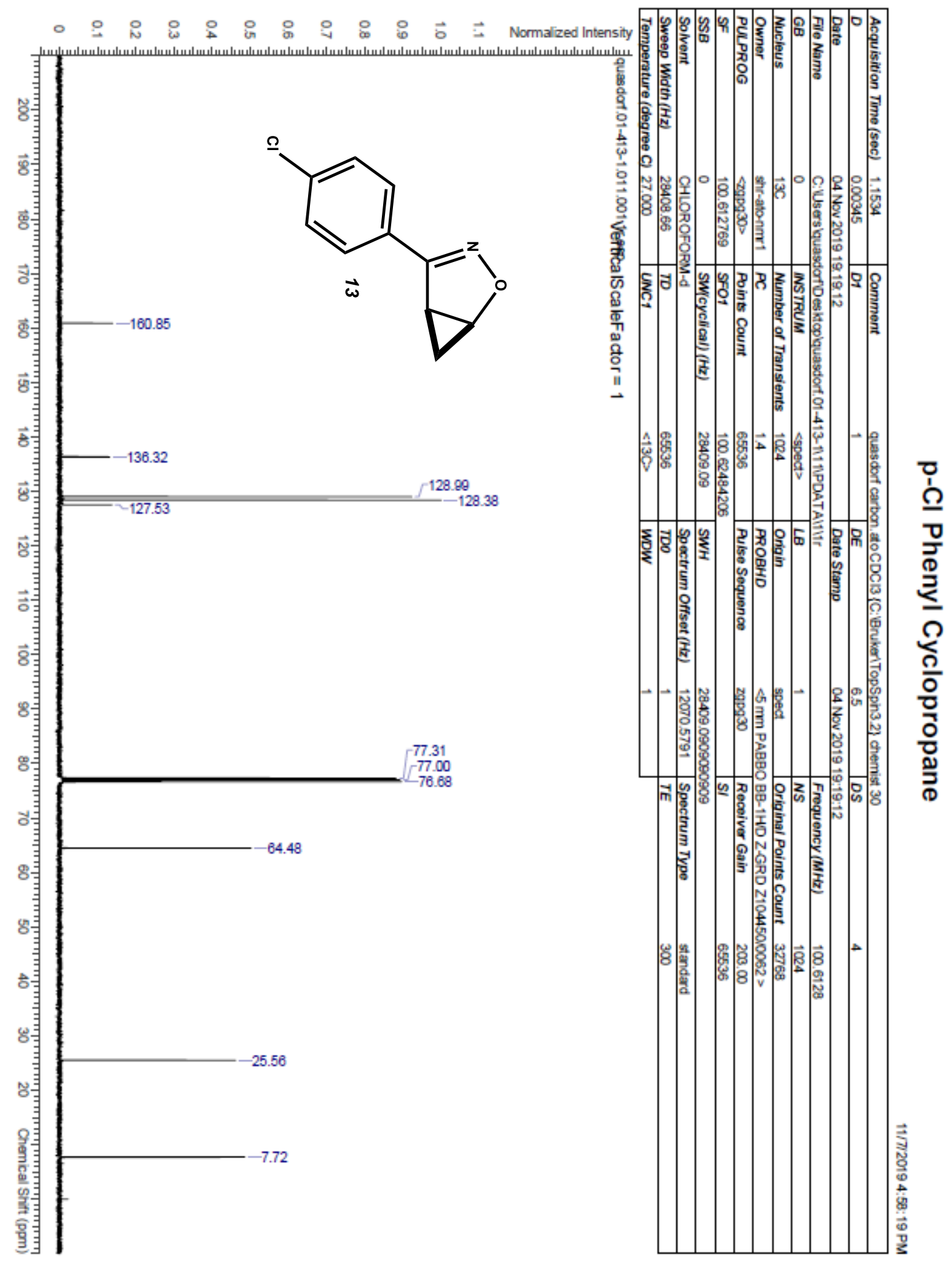




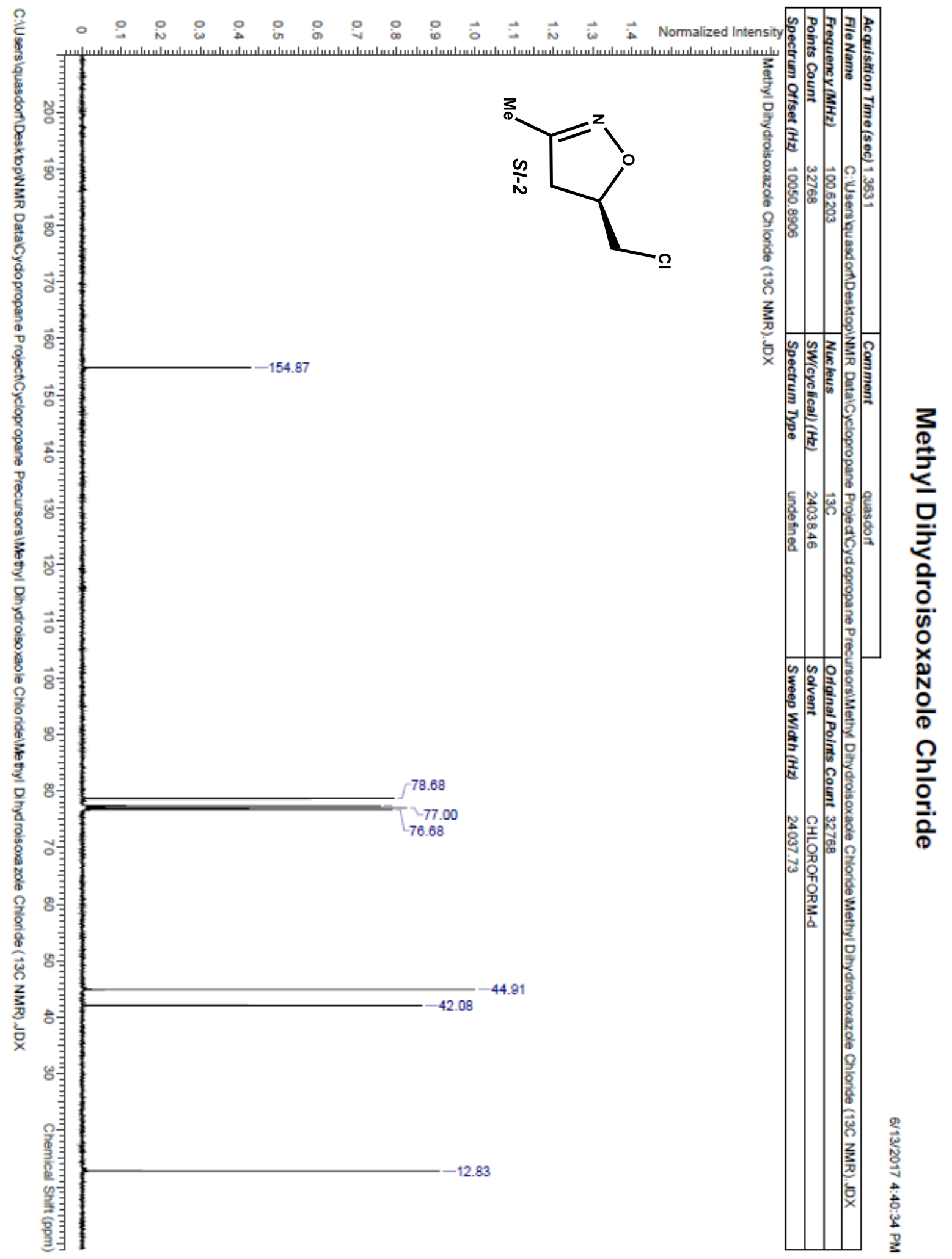




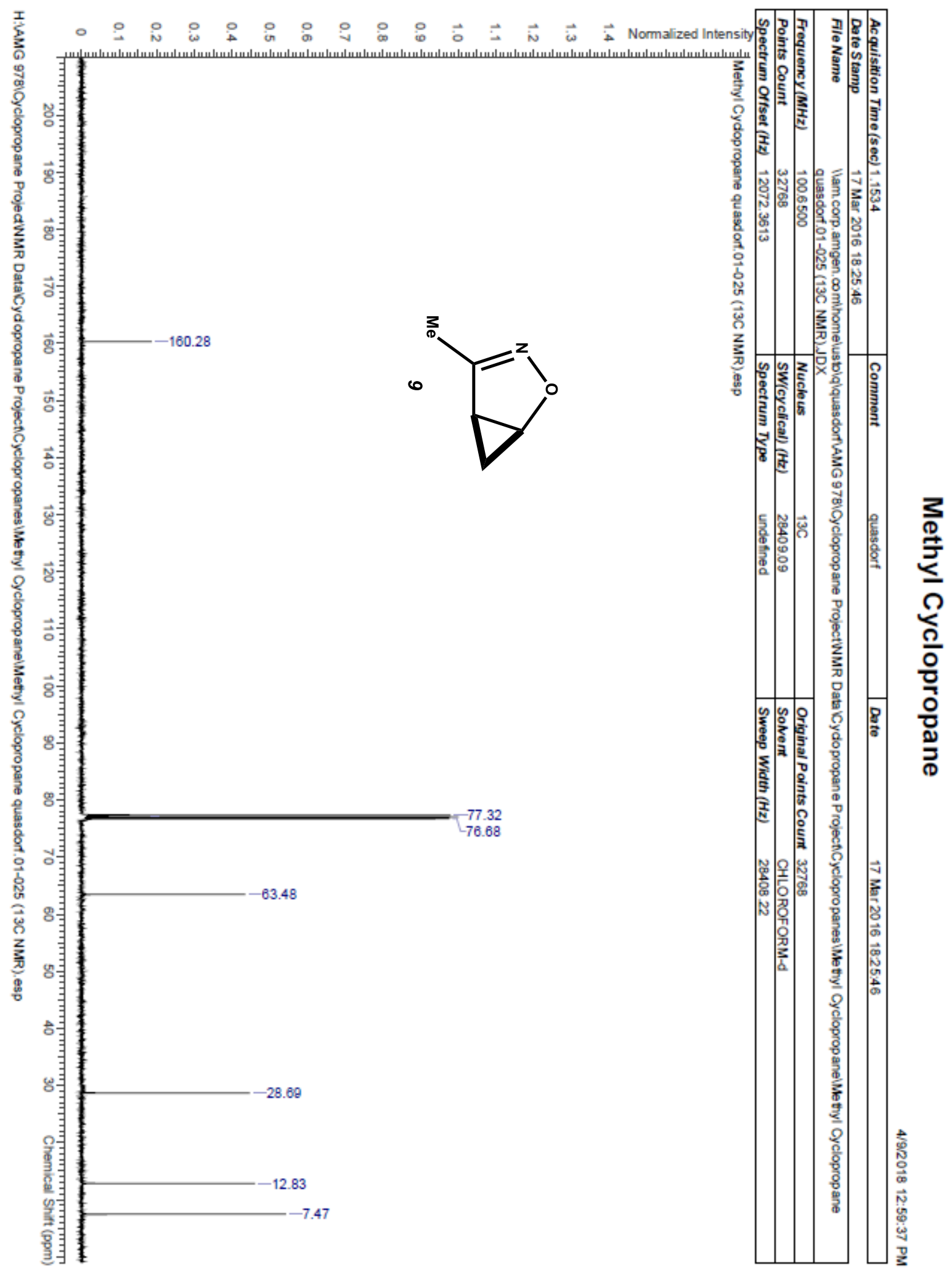




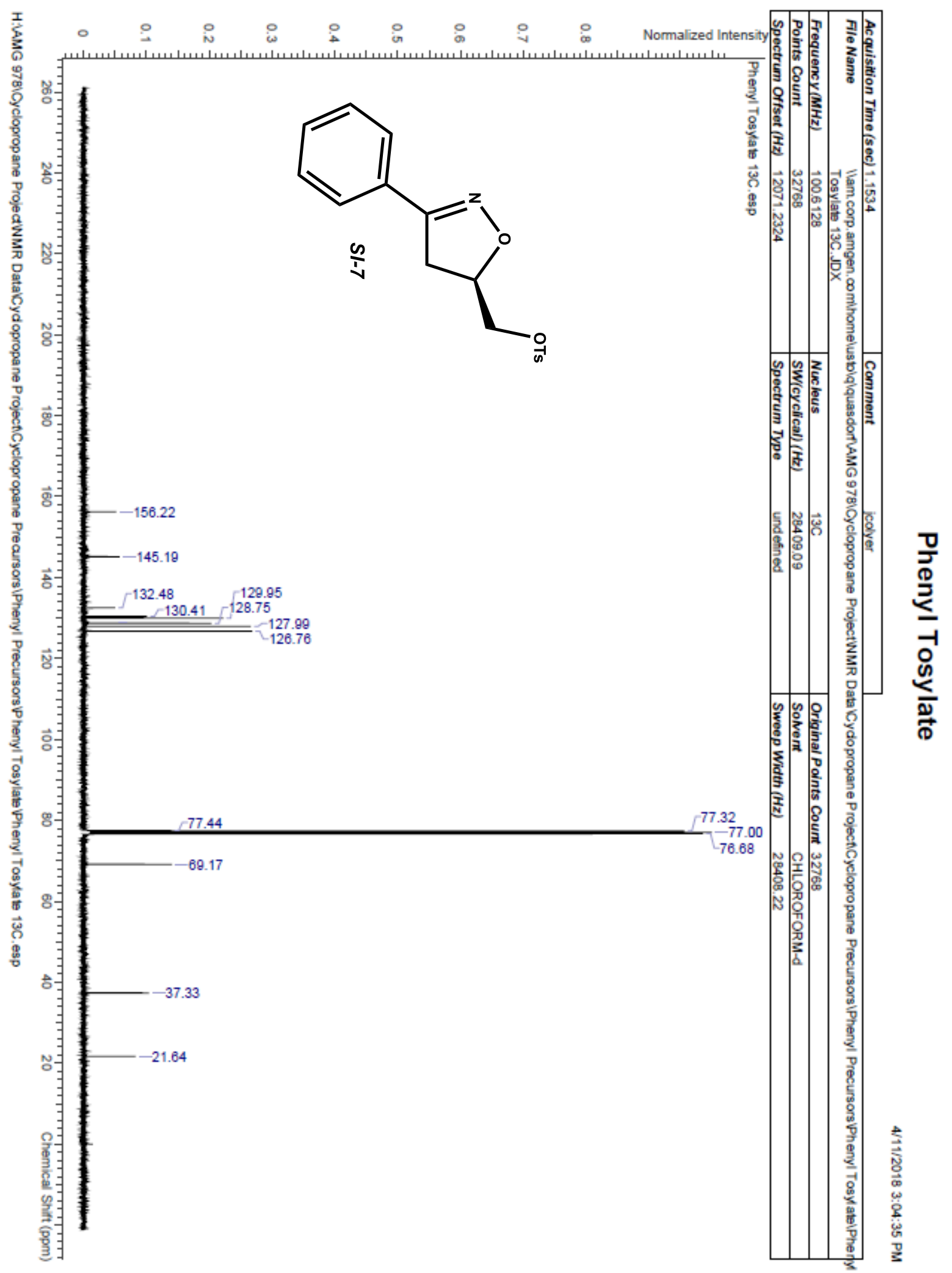




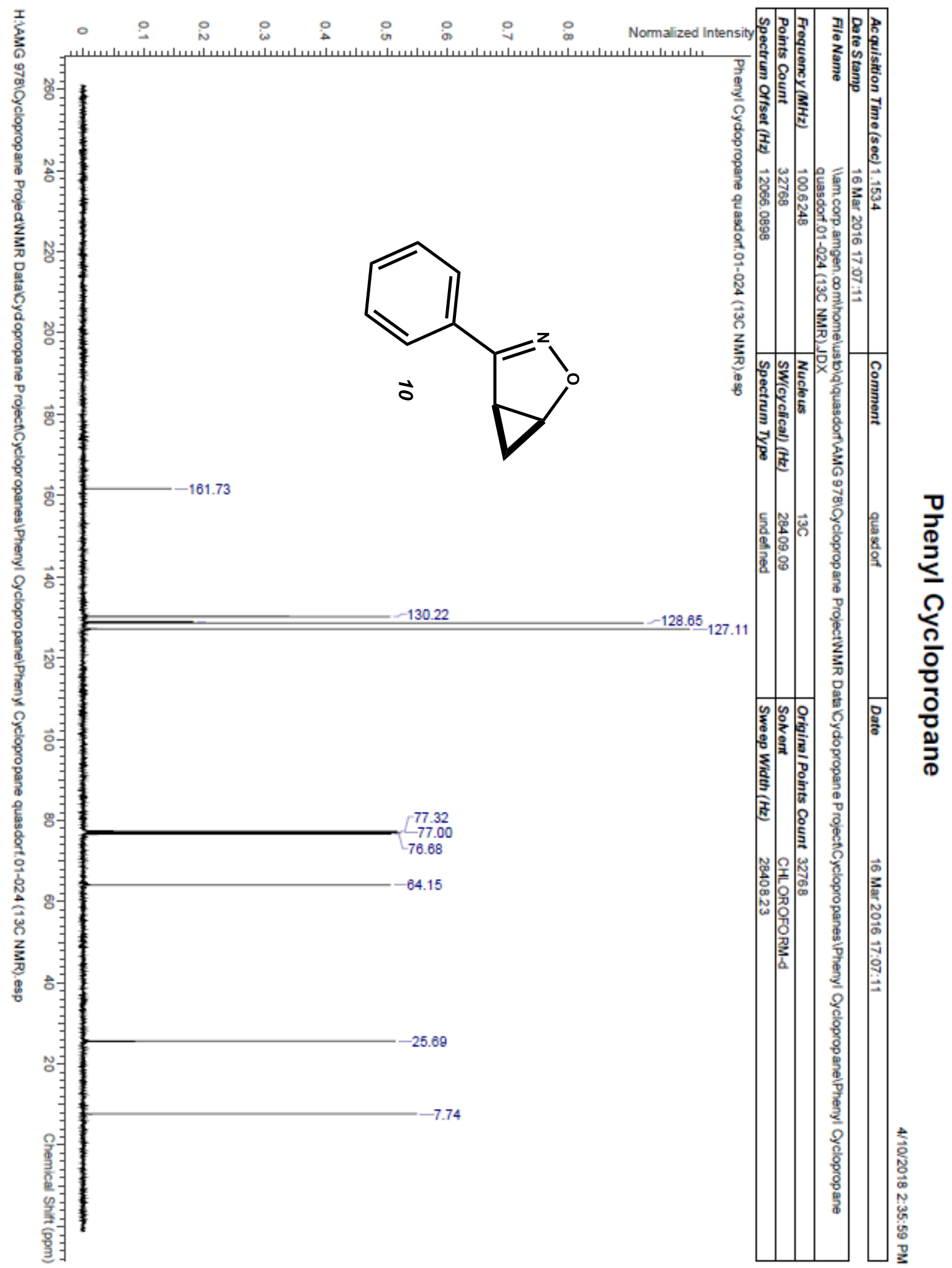




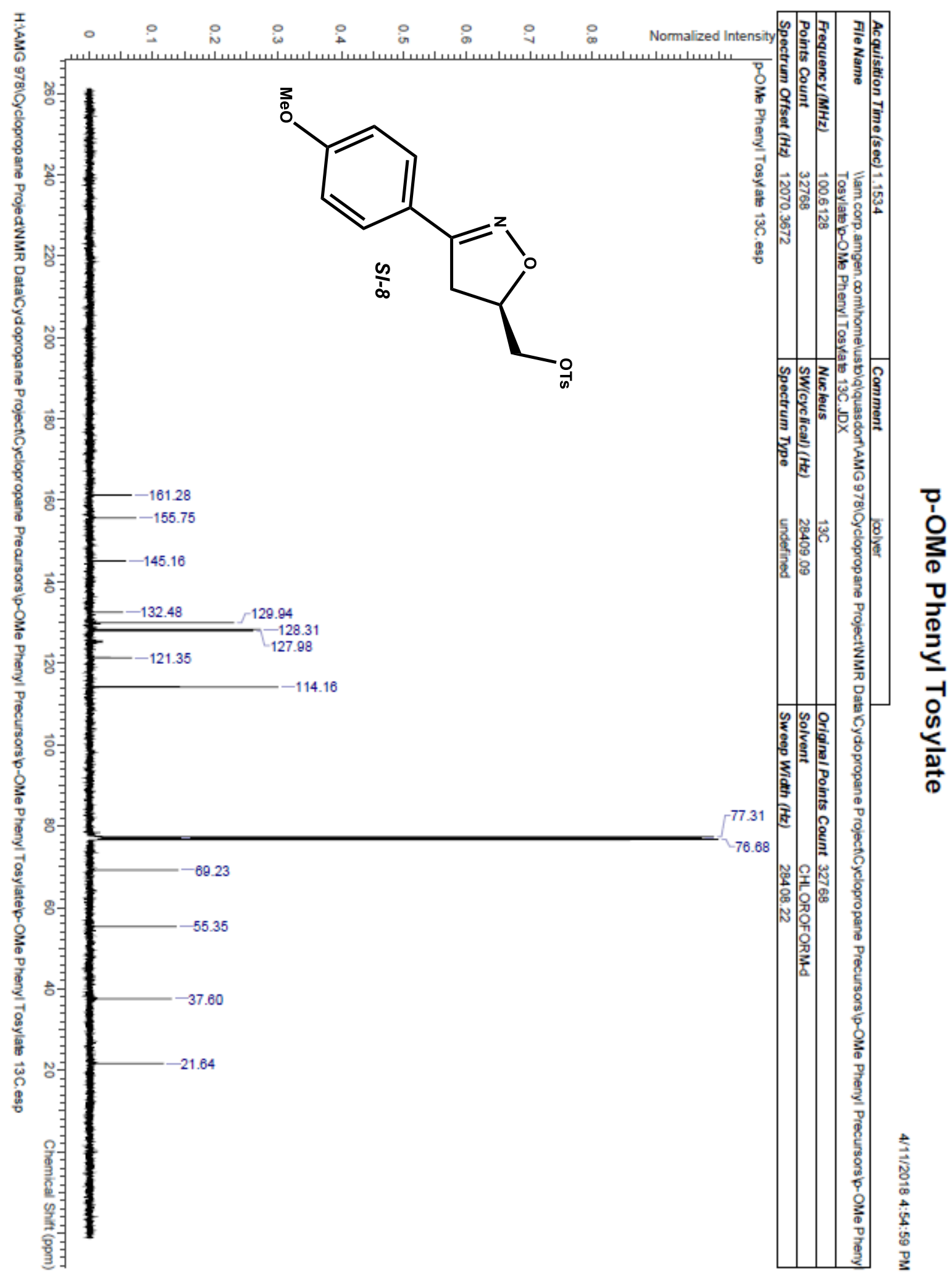




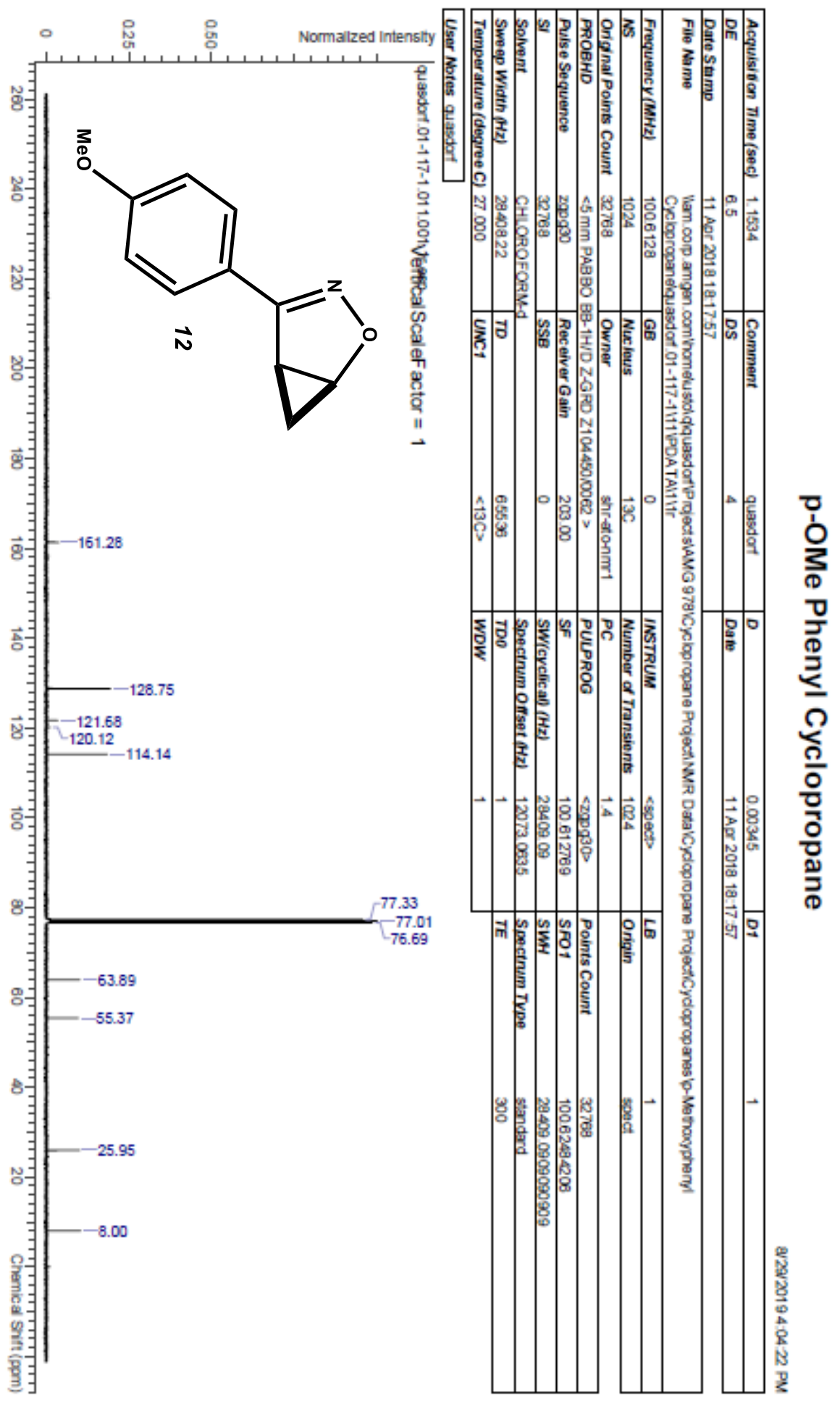




\section{${ }^{19}$ F NMR Spectra}




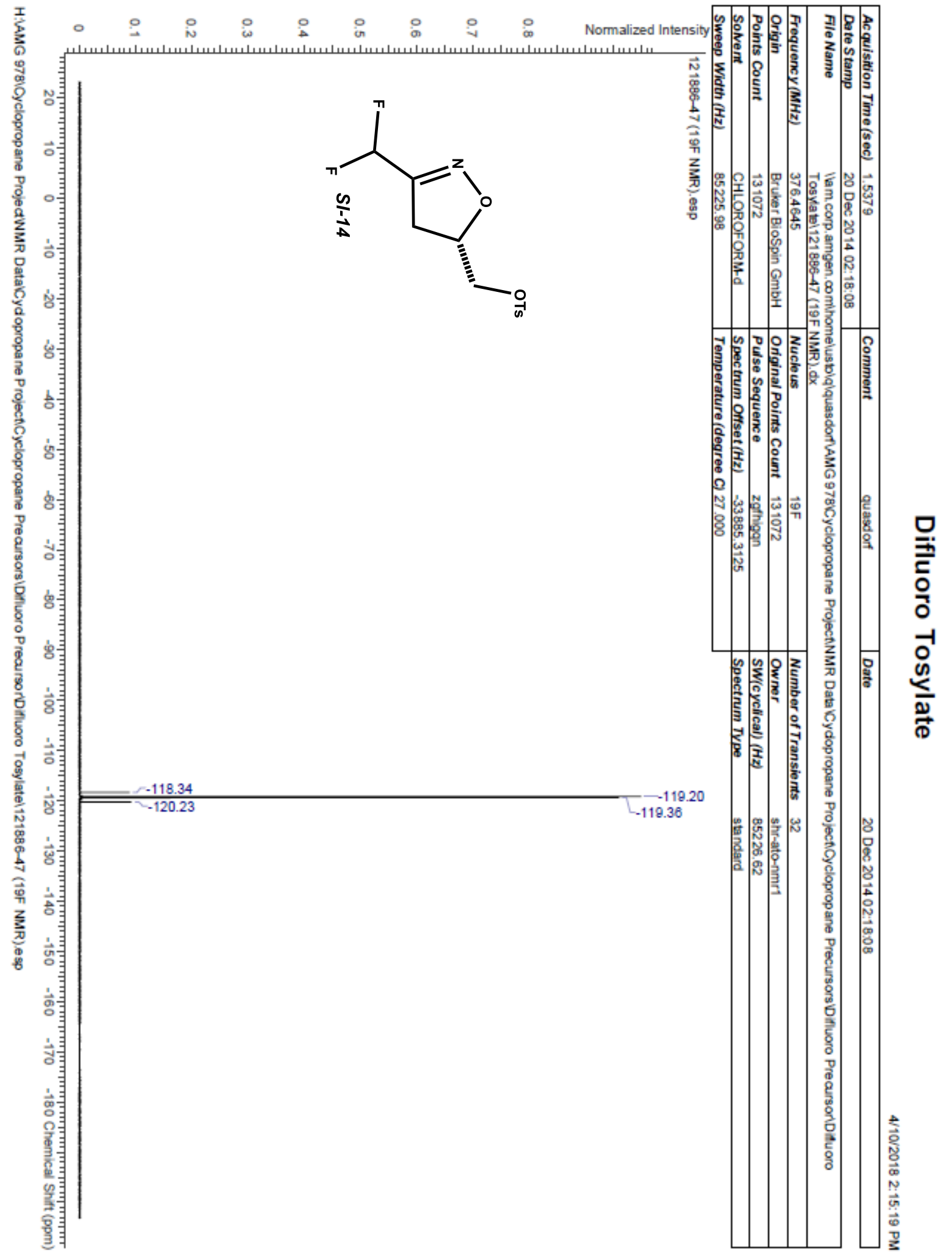




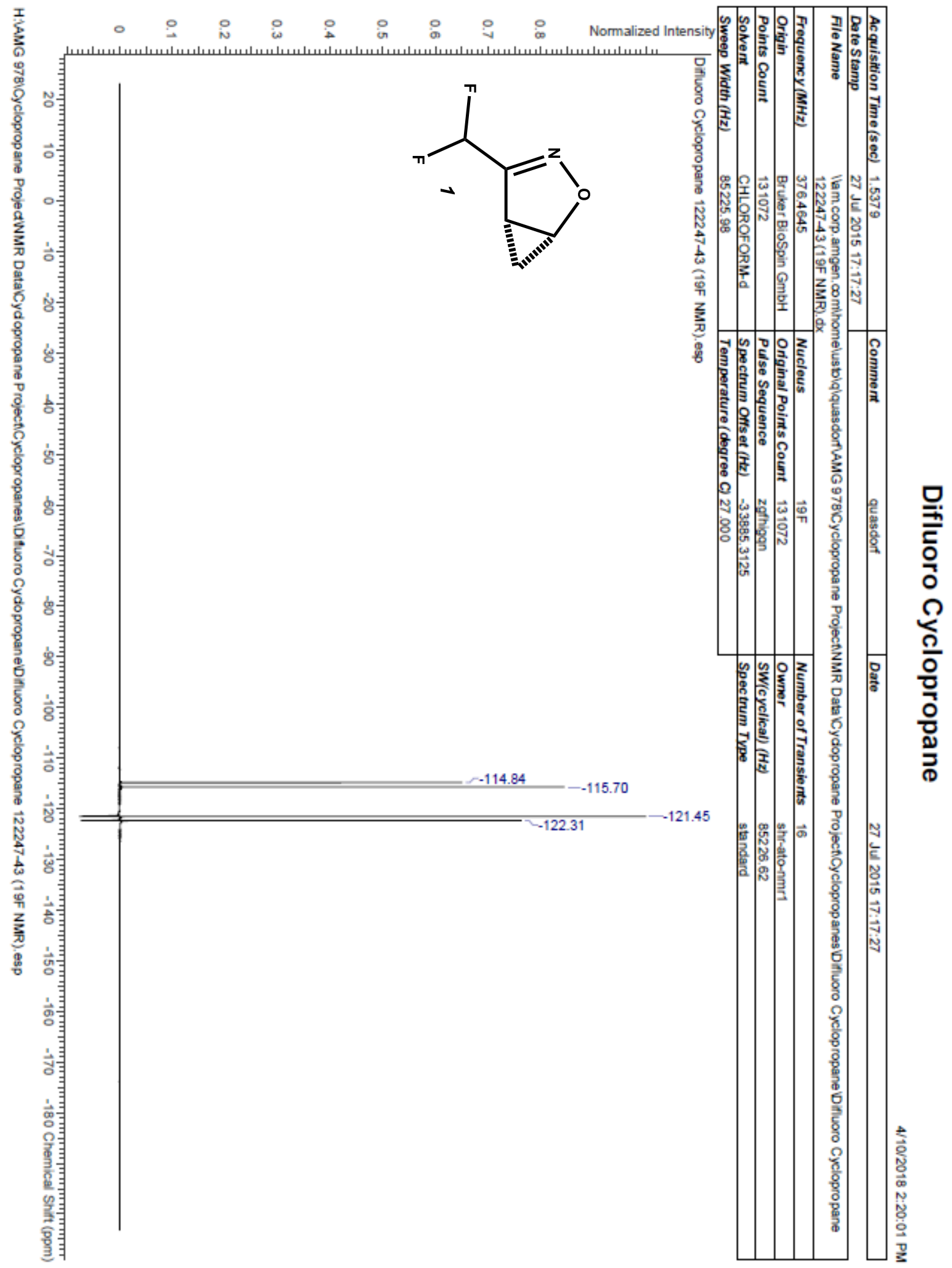




\section{Computational Data}

\section{A. Discussion}

All calculations were performed with the Gaussian 16 program [Gaussian 16, Revision C.01, M. J. Frisch, G. W. Trucks, H. B. Schlegel, G. E. Scuseria, M. A. Robb, J. R. Cheeseman, G. Scalmani, V. Barone, G. A. Petersson, H. Nakatsuji, X. Li, M. Caricato, A. V. Marenich, J. Bloino, B. G. Janesko, R. Gomperts, B. Mennucci, H. P. Hratchian, J. V. Ortiz, A. F. Izmaylov, J. L. Sonnenberg, D. WilliamsYoung, F. Ding, F. Lipparini, F. Egidi, J. Goings, B. Peng, A. Petrone, T. Henderson, D. Ranasinghe, V. G. Zakrzewski, J. Gao, N. Rega, G. Zheng, W. Liang, M. Hada, M. Ehara, K. Toyota, R. Fukuda, J. Hasegawa, M. Ishida, T. Nakajima, Y. Honda, O. Kitao, H. Nakai, T. Vreven, K. Throssell, J. A. Montgomery, Jr., J. E. Peralta, F. Ogliaro, M. J. Bearpark, J. J. Heyd, E. N. Brothers, K. N. Kudin, V. N. Staroverov, T. A. Keith, R. Kobayashi, J. Normand, K. Raghavachari, A. P. Rendell, J. C. Burant, S. S. Iyengar, J. Tomasi, M. Cossi, J. M. Millam, M. Klene, C. Adamo, R. Cammi, J. W. Ochterski, R. L. Martin, K. Morokuma, O. Farkas, J. B. Foresman, and D. J. Fox, Gaussian, Inc., Wallingford CT, 2016.].

Initial CASSCF and DFT calculations were carried out in a manner as analogous as possible to that described in previous work by Houk [Suhrada. C. P. et. al, J. Am. Chem. Soc. 2002, 124, 8796-8797].

\section{B. CASSCF Results}

To distinguish between the singlet diradical and dipolar pathways, a CASSCF(4,4)/6-31+G(d) optimization was carried out beginning with the closed-shell DFT transition structure for epimerization of cyclopropane 1, with 4 electrons in 4 orbitals as the active space. The orbitals were selected by running a natural orbital analysis on the broken spin-symmetry unrestricted Hartree-Fock wavefunction and including any occupied natural orbitals with an occupancy less than 1.9, and any virtual natural orbitals with an occupancy greater than 0.1. Optimized orbital images and occupancies of the CASSCF $(4,4) / 6$ 31+G(d) transition structure for $\mathbf{1}$ are shown below. A similar process was carried out for 3difluoromethyl bicyclo[3.1.0]hex-2-ene (direct analog of cyclopropane 1 possessing an all-carbon core), and an example of a "polarized diradical" from the literature, the nitroso intermediate species $4 \mathbf{a}$ from [Leach et. al. J. Am. Chem. Soc. 2002, 124 14820-14821]), for comparison.

The optimized orbitals for the transition structures for both cyclopropane $\mathbf{1}$ and its hydrocarbon parent are shown in the figure below. While antibonding interactions between the oxygen p orbital and what would be the $\mathrm{N}-\mathrm{C}$ sigma orbital in cyclopropane $\mathbf{1}$ break the core symmetry that exists in the all-carbon variant, the orbitals are otherwise remarkably similar. 

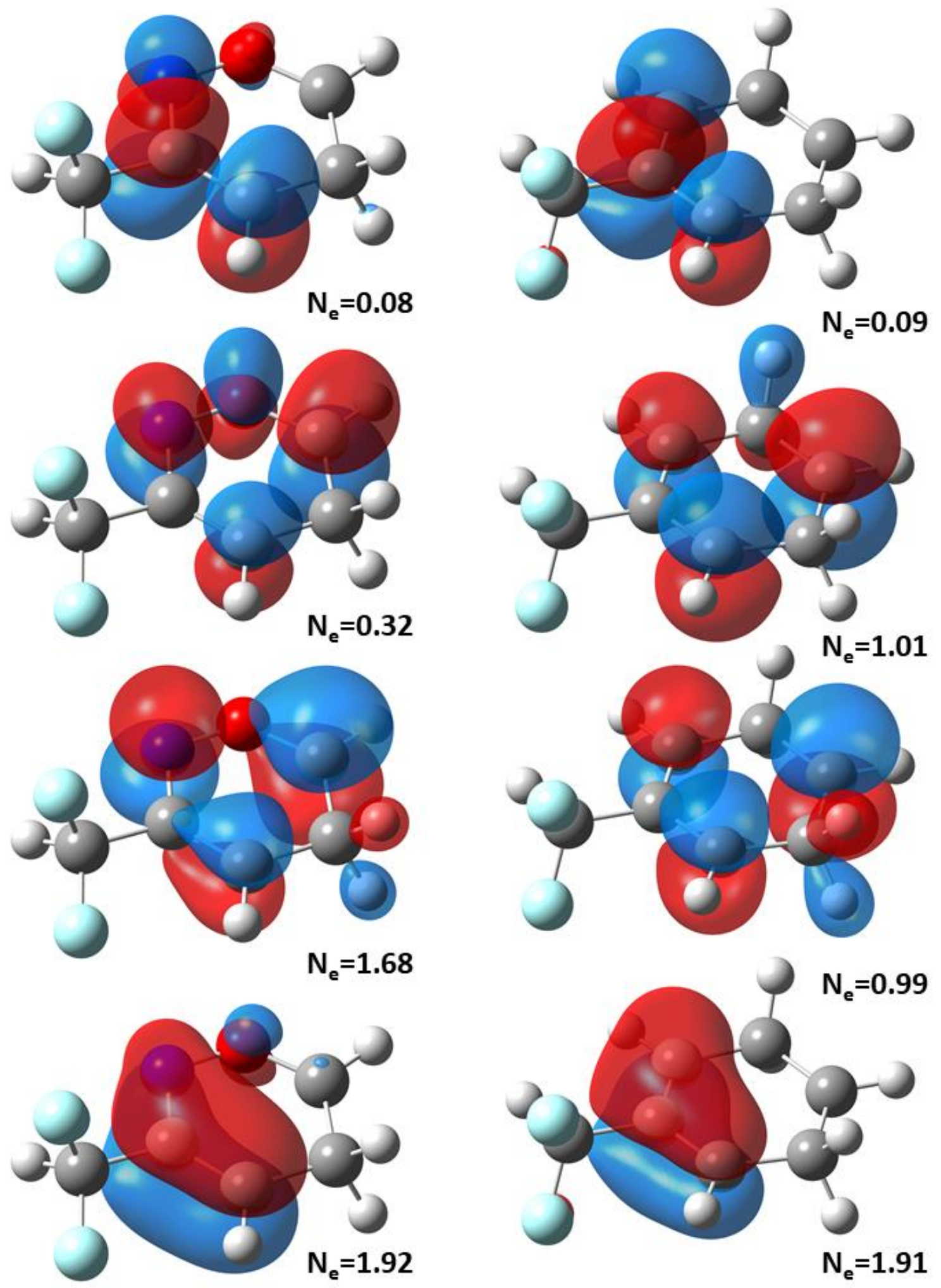
CAS $(4,4) / 6-31+\mathrm{G}^{*}$ active space orbitals and occupancies (at the 0.04 isocontour level) for the ringopening transition structures of $\mathbf{1}$ (left) and all-carbon-core analog (right), illustrating the similarities in orbital topology yet differences in occupancy.

The resulting CAS occupancies were 1.92, 1.68, 0.32 and 0.08 at the ring opening TS of 1, 1.91, 1.01, 0.99, 0.09 for the corresponding TS of 3-difluoromethyl bicyclo[3.1.0]hex-2-ene, and 1.99, 1.22, 0.78 and 0.01 for the HNO / propene adduct intermediate 4a. These results indicate that although there exists a slight multi-reference character associated with the ring opening TS of $\mathbf{1}$, the resultant optimized active space (along with the results of the DFT stability analysis) yields a picture more consistent with a dipolar electronic structure than a diradical (or even a polarized diradical) one.

Orbital selection for the CAS calculation on the reactant of 1 was carried out by first localizing RHF/6$31+\mathrm{G}(\mathrm{d})$ orbitals by the natural bond orbital method for the occupied space, and natural localized molecular orbital method for the virtual space. From these localized orbitals the $\mathrm{C}=\mathrm{N} \pi / \pi^{*}$ and cyclopropyl C-C $\sigma / \sigma^{*}$ were visually identified and used for the initial CAS guess. Optimized CAS orbitals are shown below.
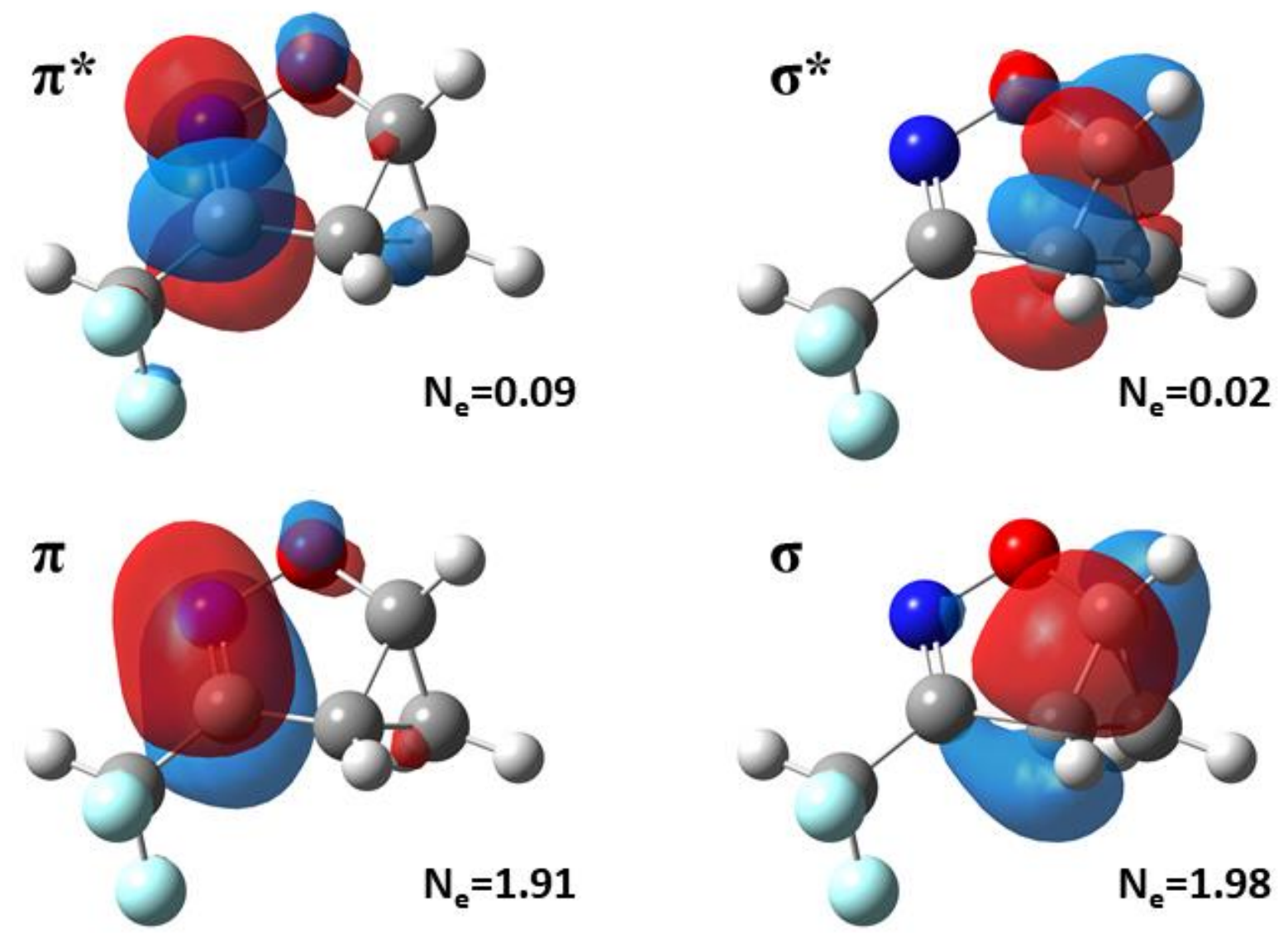
CAS $(4,4) / 6-31+\mathrm{G}^{*}$ active space orbitals and occupations for reactant $\mathbf{1}$.

\section{DFT Results}

Preliminary calculations in this study utilized the B3LYP/6-311+G(d,p) functional and basis and the IEFPCM solvation method. All solvents except for MTBE were available using built-in parameters using the corresponding solvent keyword in Gaussian. MTBE calculations were carried out using a generic solvent with parameters found in the Handbook of Organic Solvent Properties [Smallwood, I.M. Butterworth-Heinemann, 1996] $\left(\varepsilon=4.5, \varepsilon_{\infty}=1.874\right)$. Minimum energy structures of the reactant and the ring-opened transition structure on the electronic singlet surface were located for each of the cyclopropane species studied in the paper (transition structure displacement vectors shown below; see also animated GIF included in SI). The transition structures were confirmed through IRC analysis to be those corresponding to the isomerization reaction. All transition structures were found to have stable closed shell DFT densities. A geometry optimization was also carried out on the triplet diradical surface beginning with the closed shell singlet transition state geometry, finding a slightly distorted open-ring intermediate with all positive eigenvalues in the Hessian and an energy approx. $10 \mathrm{kcal} / \mathrm{mol}$ higher than the closed shell transition state at the same level of theory.

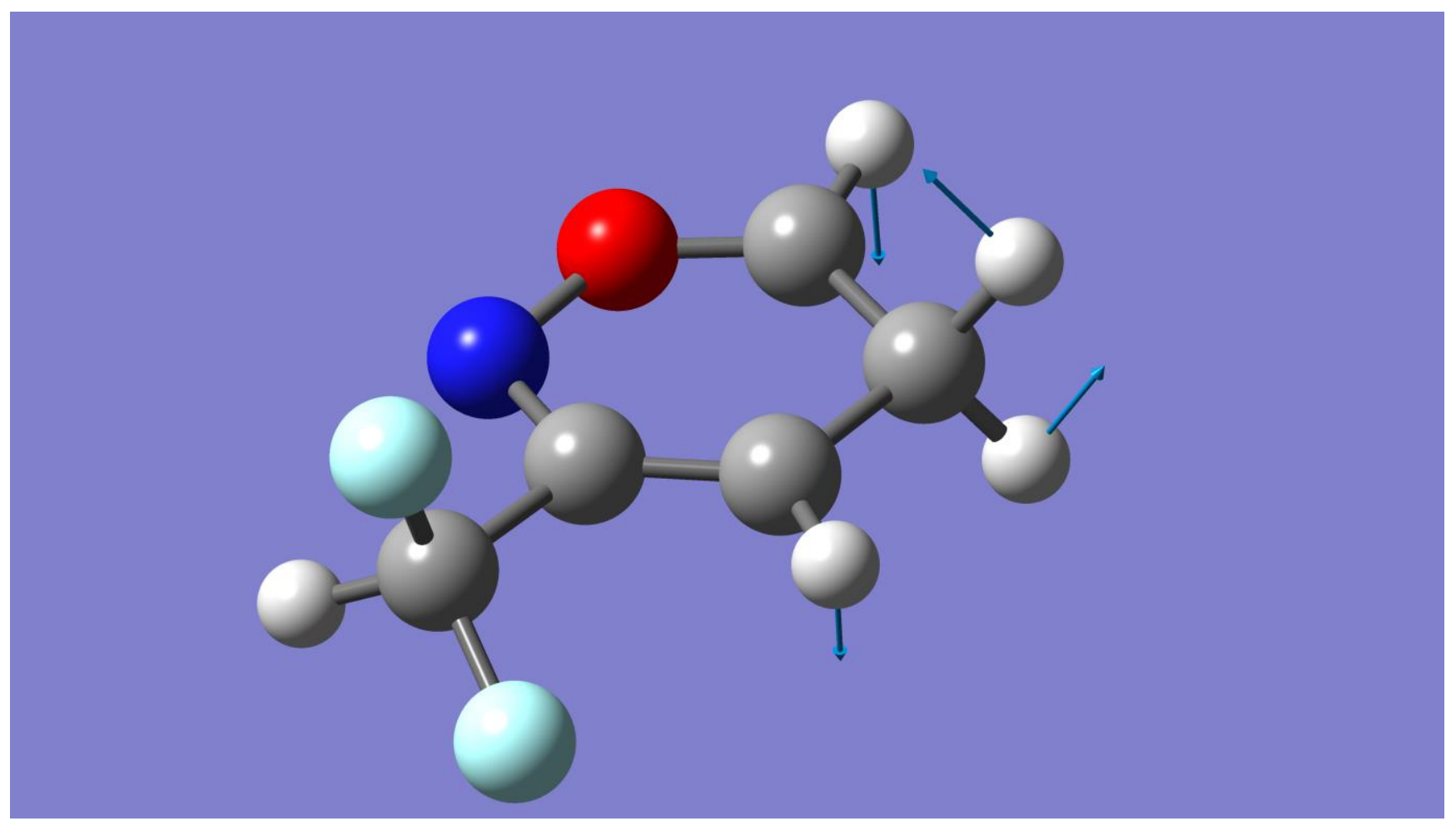

The barriers for all 8 cyclopropane species were computed in acetonitrile, and strong correlation $\left(\mathrm{R}^{2}=\right.$ 0.93, Slope $=1.08)$ was observed between the B3LYP/6-311+G(d,p) and the experimentally determined 
free energies. The resulting barrier heights were, however, systematically underestimated when compared to experiment. To investigate if this systematic error was due to choice of functional or basis set, a selection of functionals that were previously demonstrated [Mardirossian, M. et. al. Mol. Phys. 2017, 115, 2315-2372 and Goerigk L. et. al. Phys. Chem. Chem. Phys. 2017, 19, 32184-32215] to perform well for pericyclic reactions were investigated: $\omega \mathrm{B} 97 \mathrm{X}-\mathrm{D}, \mathrm{M} 06-2 \mathrm{X}$ and M08-HX, with the def2-TZVPPD basis set used to test basis set dependence.

To begin, the structures were optimized using $\omega \mathrm{B} 97 \mathrm{X}-\mathrm{D} / 6-311+\mathrm{G}(\mathrm{d}, \mathrm{p})$, and frequency calculations were carried out to verify the correct number of imaginary frequencies for each stationary point, and to estimate the free energies at the temperature of interest. Energy calculations were carried out at the resultant geometries for each of the remaining 5 model chemistries, with free energies estimated by adding the wB97X-D/6-311+G(d,p) thermal corrections (i.e. free energy minus total electronic + nuclear energy). This data, along with the initial B3LYP/6-311+G(d,p) results, is summarized in the tables in the following section. The frequency scale factors for B3LYP and $\omega$ B97X-D were taken from the database of frequency scale factors [I.M. Alecu et. al. J. Chem. Theory Comput. 2010, 6, 2872-2887].

All DFT methods showed strong correlation with experiment, with all methods but B3LYP achieving an $\mathrm{R}^{2}$ greater than 0.9 before thermal correction. However, the computed free energies are under/over estimated by a constant $2-5 \mathrm{kcal} / \mathrm{mol}$ depending on the functional. In an attempt to determine to what extent this error was inherent to the use of DFT energies, CAS-MP2 energies were computed at the CAS $(4,4)$ optimized geometries, and $\operatorname{CCSD}(\mathrm{T})$ energies were computed at the $\omega \mathrm{B} 97 \mathrm{X}-\mathrm{D}$ optimized geometries for cyclopropane 1 .

\section{Reactant (Eh) Transition State (Eh) Barrier (kcal/mol)}

\begin{tabular}{|c|c|c|c|}
\hline $\begin{array}{r}C A S-M P 2 / \text { aug-cc- } p V T Z \\
/ / C A S(4,4) / 6-31+G(d)\end{array}$ & -522.40353 & -522.34146 & 35.86 \\
\hline $\begin{array}{r}C C S D(T) / a u g-c c-p V T Z \\
/ / \omega B 97 X-D / 6-311+G(d, p)\end{array}$ & -522.39491 & -522.34639 & 30.45 \\
\hline
\end{tabular}

The difference between the barrier computed using the two electronic structure methods and the same barrier computed at each of the DFT model chemistries is plotted below, alongside each functional's observed mean signed error relative to experiment for the 8 cyclopropanes studied in this manuscript. For $\omega$ B97X-D and M06-2X, the error in the DFT barrier height relative to the CCSD(T) reference for 1, and the mean signed error in the DFT barrier heights for all 8 cyclopropane substrates relative to 
experiment, agree to within $1 \mathrm{kcal} / \mathrm{mol}$ of one another. This suggests that $\mathrm{CCSD}(\mathrm{T})$ calculations carried out on the DFT optimized structures would show excellent agreement to the experimental results, and serves to further validate that the closed-shell DFT structures and observed energetic trends are correct.

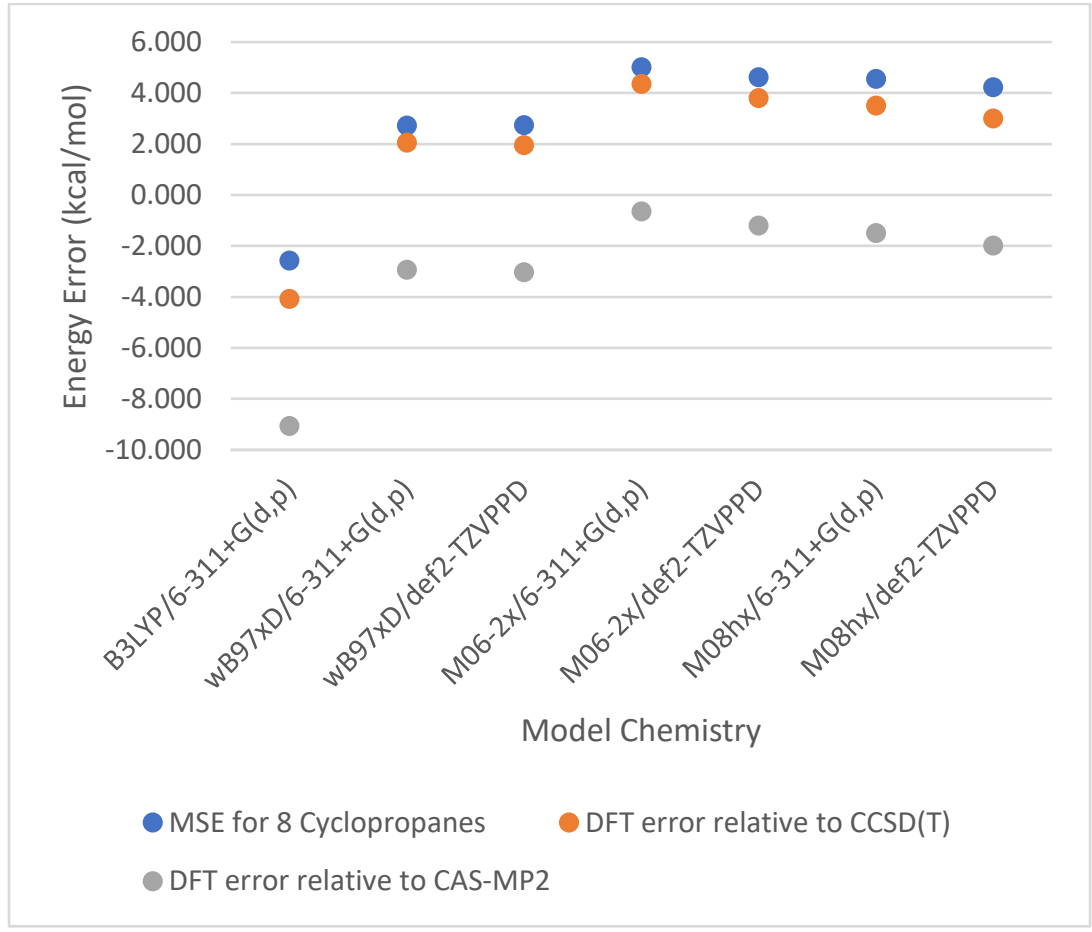

While all tested model chemistries are likely suitable for studying this reaction, free energies computed by $\omega \mathrm{B} 97 \mathrm{X}-\mathrm{D} / 6-311+\mathrm{G}(\mathrm{d}, \mathrm{p})$ were ultimately used in the manuscript due to a favorable combination of correlation to experiment and minimum error (among the methods tested) in barrier heights compared to both experiment and $\operatorname{CCSD}(\mathrm{T})$ calculations.

We also investigated the effect of both solvent polarity and substituent on dipolar character at the transition state by comparing the dipole moment magnitude at the TS to that of the reactant $(\Delta$ in the table below). These data support the view that increasing solvent polarity and electron withdrawing character of the substrate both have the effect of stabilizing dipole formation at the transition state, and accelerating the rate of racemization. 
TABLE: Calculated dipole moment magnitudes versus changes in solvent polarity and cyclopropane substrate

\begin{tabular}{|c|c|c|c|c|c|c|c|c|c|c|c|}
\hline \multirow[b]{2}{*}{ Substrate } & \multirow[b]{2}{*}{ Solvent } & \multirow[b]{2}{*}{$\varepsilon$} & \multicolumn{3}{|c|}{ dipole (Debye) } & \multirow[b]{2}{*}{ Substrate } & \multirow[b]{2}{*}{ Solvent } & \multirow[b]{2}{*}{$\varepsilon$} & \multicolumn{3}{|c|}{ dipole (Debye) } \\
\hline & & & reactant & TS & $\Delta$ & & & & reactant & TS & $\Delta$ \\
\hline \multirow{7}{*}{$\mathrm{CF}_{2} \mathrm{H}$} & None & 1.0 & 2.6 & 5.0 & 2.4 & $\mathrm{CF}_{2} \mathrm{H}$ & \multirow{8}{*}{$\mathrm{ACN}$} & \multirow{8}{*}{35.7} & 3.2 & 7.2 & 4.0 \\
\hline & Toluene & 2.4 & 2.8 & 6.0 & 3.2 & Benzothiazole & & & 3.3 & 5.2 & 1.9 \\
\hline & MTBE & 4.5 & 3.0 & 6.5 & 3.5 & OPiv & & & 5.7 & 7.8 & 2.1 \\
\hline & THF & 7.6 & 3.1 & 6.8 & 3.7 & p-CN & & & 6.8 & 11.4 & 4.6 \\
\hline & Acetone & 20.7 & 3.1 & 7.1 & 4.0 & p-Cl & & & 4.9 & 7.9 & 3.0 \\
\hline & $\mathrm{ACN}$ & 35.7 & 3.2 & 7.2 & 4.0 & Methyl & & & 5.2 & 5.3 & 0.1 \\
\hline & DMSO & 56.7 & 3.2 & 7.2 & 4.0 & Phenyl & & & 5.0 & 5.7 & 0.7 \\
\hline & & & & & & p-OMe & & & 4.0 & 4.4 & 0.4 \\
\hline
\end{tabular}


ENERGIES: IEFPCM-B3LYP/6-311+G(D,P)

GEOMETRIES, FREQUENCIES, ZPE, THERMAL CORRECTIONS: IEFPCM-B3LYP/6-311+G(D,P)

\begin{tabular}{|c|c|c|c|c|c|c|c|c|c|c|}
\hline \multirow[b]{2}{*}{ CPD \# } & \multirow[b]{2}{*}{ R-Group } & \multirow[b]{2}{*}{ Solvent } & \multirow{2}{*}{$\begin{array}{l}\text { Minimum } \\
\text { Energy (Eh) }\end{array}$} & \multirow{2}{*}{$\begin{array}{c}\text { TS } \\
\text { Energy (Eh) }\end{array}$} & \multirow{2}{*}{$\begin{array}{c}\text { E Barrier } \\
\text { (kcal/mol) }\end{array}$} & \multicolumn{3}{|c|}{ Free Energy (Freq. Scale=0.988) } & \multirow{2}{*}{$\begin{array}{l}\text { G Barrier } \\
\text { (kcal/mol) }\end{array}$} & \multirow{2}{*}{$\begin{array}{c}\text { (G-E) Thermal } \\
\text { Correction }\end{array}$} \\
\hline & & & & & & Temp C & Min (Eh) & TS (Eh) & & \\
\hline 1 & $\mathrm{CF} 2 \mathrm{H}$ & acn & -523.25830 & -523.21950 & 24.35 & 45 & -523.19692 & -523.15960 & 23.27 & -1.08 \\
\hline 1 & $\mathrm{CF} 2 \mathrm{H}$ & dmso & -523.25839 & -523.21965 & 24.31 & 45 & -523.19679 & -523.15976 & 23.24 & -1.07 \\
\hline 1 & $\mathrm{CF} 2 \mathrm{H}$ & mtbe & -523.25593 & -523.21572 & 25.23 & 45 & -523.19420 & -523.15568 & 24.17 & -1.06 \\
\hline 1 & $\mathrm{CF} 2 \mathrm{H}$ & thf & -523.25693 & -523.21728 & 24.88 & 45 & -523.19525 & -523.15723 & 23.86 & -1.03 \\
\hline 1 & $\mathrm{CF} 2 \mathrm{H}$ & toluene & -523.25400 & -523.21283 & 25.83 & 45 & -523.19218 & -523.15277 & 24.73 & -1.11 \\
\hline 1 & $\mathrm{CF} 2 \mathrm{H}$ & none & -523.24999 & -523.20729 & 26.79 & 45 & -523.18803 & -523.14715 & 25.65 & -1.14 \\
\hline 1 & $\mathrm{CF} 2 \mathrm{H}$ & $\mathrm{ACN}$ & -523.25830 & -523.21950 & 24.35 & 30 & -523.19459 & -523.15750 & 23.27 & -1.07 \\
\hline 7 & Benzothiazole & $\mathrm{ACN}$ & -1007.00808 & -1006.96683 & 25.89 & 30 & -1006.88145 & -1006.84263 & 24.36 & -1.53 \\
\hline 8 & OPiv & $\mathrm{ACN}$ & -670.64022 & -670.59962 & 25.48 & 30 & -670.44763 & -670.40816 & 24.77 & -0.71 \\
\hline 9 & Methyl & $\mathrm{ACN}$ & -324.72350 & -324.68003 & 27.28 & 70 & -324.64713 & -324.60557 & 26.08 & -1.19 \\
\hline 10 & Phenyl & $\mathrm{ACN}$ & -516.50756 & -516.46288 & 28.04 & 70 & -516.38531 & -516.34230 & 26.99 & -1.05 \\
\hline 11 & $\mathrm{p}-\mathrm{CN}$ & $\mathrm{ACN}$ & -608.77793 & -608.73498 & 26.95 & 70 & -608.66043 & -608.61912 & 25.93 & -1.02 \\
\hline 12 & $\mathrm{p}-\mathrm{OCH} 3$ & $\mathrm{ACN}$ & -631.06693 & -631.02132 & 28.62 & 70 & -630.91748 & -630.87358 & 27.55 & -1.07 \\
\hline 13 & $\mathrm{p}-\mathrm{Cl}$ & $\mathrm{ACN}$ & -976.13025 & -976.08624 & 27.62 & 70 & -976.02027 & -975.97793 & 26.57 & -1.05 \\
\hline
\end{tabular}


ENERGIES: IEFPCM- $\omega$ B97XD/6-311+G(D,P) GEOMETRIES, FREQUENCIES, ZPE, THERMAL CORRECTIONS: IEFPCM- $\omega$ B97XD/6-311+G(D,P)

\begin{tabular}{|c|c|c|c|c|c|c|c|c|c|c|}
\hline \multirow[b]{2}{*}{ CPD \# } & \multirow[b]{2}{*}{ R-Group } & \multirow[b]{2}{*}{ Solvent } & \multirow{2}{*}{$\begin{array}{l}\text { Minimum } \\
\text { Energy (Eh) }\end{array}$} & \multirow{2}{*}{$\begin{array}{c}\text { TS } \\
\text { Energy (Eh) }\end{array}$} & \multirow{2}{*}{$\begin{array}{l}\text { E Barrier } \\
\text { (kcal/mol) }\end{array}$} & \multicolumn{3}{|c|}{ Free Energy (Freq. Scale=0.979) } & \multirow{2}{*}{$\begin{array}{l}\text { G Barrier } \\
\text { (kcal/mol) }\end{array}$} & \multirow{2}{*}{$\begin{array}{c}\text { (G-E) Thermal } \\
\text { Correction }\end{array}$} \\
\hline & & & & & & Temp C & Min (Eh) & TS (Eh) & & \\
\hline 1 & $\mathrm{CF} 2 \mathrm{H}$ & acn & -523.09526 & -523.04760 & 29.91 & 45 & -523.03306 & -522.98704 & 28.88 & -1.03 \\
\hline 1 & $\mathrm{CF} 2 \mathrm{H}$ & dmso & -523.09535 & -523.04776 & 29.86 & 45 & -523.03315 & -522.98722 & 28.83 & -1.04 \\
\hline 1 & $\mathrm{CF} 2 \mathrm{H}$ & mtbe & -523.09294 & -523.04356 & 30.98 & 45 & -523.03062 & -522.98292 & 29.93 & -1.05 \\
\hline 1 & $\mathrm{CF} 2 \mathrm{H}$ & thf & -523.09392 & -523.04522 & 30.56 & 45 & -523.03163 & -522.98458 & 29.53 & -1.03 \\
\hline 1 & $\mathrm{CF} 2 \mathrm{H}$ & toluene & -523.09103 & -523.04047 & 31.73 & 45 & -523.02862 & -522.97974 & 30.68 & -1.05 \\
\hline 1 & $\mathrm{CF} 2 \mathrm{H}$ & none & -523.08709 & -523.03462 & 32.92 & 45 & -523.02453 & -522.97381 & 31.83 & -1.10 \\
\hline 1 & $\mathrm{CF} 2 \mathrm{H}$ & $\mathrm{ACN}$ & -523.09526 & -523.04760 & 29.91 & 30 & -523.03096 & -522.98495 & 28.87 & -1.04 \\
\hline 7 & Benzothiazole & $\mathrm{ACN}$ & -1006.77612 & -1006.72710 & 30.76 & 30 & -1006.64865 & -1006.60243 & 29.01 & -1.75 \\
\hline 8 & OPiv & $\mathrm{ACN}$ & -670.42587 & -670.37601 & 31.29 & 30 & -670.23293 & -670.18386 & 30.79 & -0.49 \\
\hline 9 & Methyl & $\mathrm{ACN}$ & -324.61375 & -324.56157 & 32.74 & 70 & -324.53703 & -324.48674 & 31.56 & -1.19 \\
\hline 10 & Phenyl & $\mathrm{ACN}$ & -516.32763 & -516.27485 & 33.12 & 70 & -516.20487 & -516.15369 & 32.12 & -1.00 \\
\hline 11 & $\mathrm{p}-\mathrm{CN}$ & $\mathrm{ACN}$ & -608.56122 & -608.51019 & 32.02 & 70 & -608.44310 & -608.39346 & 31.15 & -0.87 \\
\hline 12 & $\mathrm{p}-\mathrm{OCH} 3$ & $\mathrm{ACN}$ & -630.85104 & -630.79745 & 33.63 & 70 & -630.70071 & -630.64873 & 32.62 & -1.01 \\
\hline 13 & $\mathrm{p}-\mathrm{Cl}$ & $\mathrm{ACN}$ & -975.93500 & -975.88297 & 32.65 & 70 & -975.82440 & -975.77385 & 31.72 & -0.93 \\
\hline 1 & $\mathrm{CF} 2 \mathrm{H}$ & MTBE & -523.09294 & -523.04356 & 30.98 & 30 & -523.02852 & -522.98082 & 29.93 & -1.05 \\
\hline 1 & $\mathrm{CF} 2 \mathrm{H}$ & Acetone & -523.09498 & -523.04710 & 30.05 & 30 & -523.03067 & -522.98441 & 29.03 & -1.02 \\
\hline 1 & $\mathrm{CF} 2 \mathrm{H}$ & ACN & -523.09526 & -523.04760 & 29.91 & 30 & -523.03096 & -522.98495 & 28.87 & -1.04 \\
\hline
\end{tabular}


ENERGIES: IEFPCM- $\omega$ B97XD/DEF2-TZVPPD

GEOMETRIES, FREQUENCIES, ZPE, THERMAL CORRECTIONS: IEFPCM- $\omega$ B97XD/6-311+G(D,P)

\begin{tabular}{r|ccccc|ccc}
\hline CPD \# & R-Group & Solvent & $\begin{array}{c}\text { Minimum } \\
\text { Energy (Eh) }\end{array}$ & $\begin{array}{c}\text { TS } \\
\text { Energy (Eh) }\end{array}$ & $\begin{array}{c}\text { E Barrier } \\
\text { (kcal/mol) }\end{array}$ & Temp C & $\begin{array}{c}\text { Free Energy } \\
\text { Correction }\end{array}$ & Barrier \\
\hline $\mathbf{1}$ & CF2H & acn & -523.16410 & -523.11649 & 29.88 & 45 & -1.03 & 28.85 \\
$\mathbf{1}$ & CF2H & dmso & -523.16418 & -523.11664 & 29.83 & 45 & -1.04 & 28.80 \\
$\mathbf{1}$ & CF2H & mtbe & -523.16209 & -523.11280 & 30.93 & 45 & -1.05 & 29.87 \\
$\mathbf{1}$ & CF2H & thf & -523.16293 & -523.11432 & 30.50 & 45 & -1.03 & 29.47 \\
$\mathbf{1}$ & CF2H & toluene & -523.16043 & -523.11000 & 31.65 & 45 & -1.05 & 30.60 \\
$\mathbf{1}$ & CF2H & none & -523.15703 & -523.10472 & 32.83 & 45 & -1.10 & 31.73 \\
& & & & & & & & \\
$\mathbf{1}$ & CF2H & ACN & -523.16410 & -523.11649 & 29.88 & 30 & -1.04 & 28.84 \\
$\mathbf{7}$ & Benzothizole & ACN & -1006.85895 & -1006.81002 & 30.70 & 30 & -1.75 & 28.95 \\
$\mathbf{8}$ & OPiv & ACN & -670.50815 & -670.45852 & 31.14 & 30 & -0.49 & 30.65 \\
$\mathbf{9}$ & Methyl & ACN & -324.65511 & -324.60320 & 32.57 & 70 & -1.19 & 31.38 \\
$\mathbf{1 0}$ & Phenyl & ACN & -516.39154 & -516.33880 & 33.10 & 70 & -1.00 & 32.09 \\
$\mathbf{1 1}$ & p-CN & ACN & -608.63610 & -608.58506 & 32.03 & 70 & -0.87 & \\
$\mathbf{1 2}$ & p-OCH3 & ACN & -630.93019 & -630.87652 & 33.67 & 70 & -1.01 & 31.16 \\
$\mathbf{1 3}$ & p-Cl & ACN & -976.00275 & -975.95048 & 32.80 & 70 & -0.93 & 32.66
\end{tabular}

${ }^{1}$ Free energy thermal correction from $\omega \mathrm{B} 97 \mathrm{xD} / 6-311+\mathrm{G}(\mathrm{d}, \mathrm{p})$ frequency calculation 
ENERGIES: IEFPCM-M06-2X/6-311+G(D,P)

GEOMETRIES, FREQUENCIES, ZPE, THERMAL CORRECTIONS: IEFPCM- $\omega$ B97XD/6-311+G(D,P)

\begin{tabular}{r|cc|ccc|ccc}
\hline CPD \# & R-Group & Solvent & $\begin{array}{c}\text { Minimum } \\
\text { Energy (Eh) }\end{array}$ & $\begin{array}{c}\text { TS } \\
\text { Energy (Eh) }\end{array}$ & $\begin{array}{c}\text { E Barrier } \\
\text { (kcal/mol) }\end{array}$ & Temp C & $\begin{array}{c}\text { Free Energy } \\
\text { Correction }\end{array}$ & Barrier $^{1}$ \\
\hline $\mathbf{1}$ & CF2H & acn & -523.06843 & -523.01759 & 31.91 & 45 & -1.03 & 30.87 \\
$\mathbf{1}$ & CF2H & dmso & -523.06852 & -523.01776 & 31.86 & 45 & -1.04 & 30.82 \\
$\mathbf{1}$ & CF2H & mtbe & -523.06609 & -523.01337 & 33.08 & 45 & -1.05 & 32.03 \\
$\mathbf{1}$ & CF2H & thf & -523.06707 & -523.01511 & 32.61 & 45 & -1.03 & 31.58 \\
$\mathbf{1}$ & CF2H & toluene & -523.06416 & -523.01014 & 33.90 & 45 & -1.05 & 32.85 \\
$\mathbf{1}$ & CF2H & none & -523.06017 & -523.00404 & 35.23 & 45 & -1.10 & 34.13 \\
& & & & & & & & \\
$\mathbf{1}$ & CF2H & ACN & -523.06843 & -523.01759 & 31.91 & 30 & -1.04 & 30.87 \\
$\mathbf{7}$ & Benzothizole & ACN & -1006.74106 & -1006.68864 & 32.90 & 30 & -1.75 & 31.14 \\
$\mathbf{8}$ & OPiv & ACN & -670.36269 & -670.30898 & 33.70 & 30 & -0.49 & 33.21 \\
$\mathbf{9}$ & Methyl & ACN & -324.58631 & -324.52998 & 35.35 & 70 & -1.19 & 34.16 \\
$\mathbf{1 0}$ & Phenyl & ACN & -516.29641 & -516.23974 & 35.56 & 70 & -1.00 & \\
$\mathbf{1 1}$ & p-CN & ACN & -608.53868 & -608.48384 & 34.41 & 70 & -0.87 & 34.56 \\
$\mathbf{1 2}$ & p-OCH3 & ACN & -630.81205 & -630.75465 & 36.02 & 70 & -1.01 & 33.54 \\
$\mathbf{1 3}$ & p-Cl & ACN & -975.89676 & -975.84068 & 35.19 & 70 & -0.93 & 35.01
\end{tabular}

${ }^{1}$ Free energy thermal correction from $\omega \mathrm{B} 97 \mathrm{xD} / 6-311+\mathrm{G}(\mathrm{d}, \mathrm{p})$ frequency calculation 
ENERGIES: IEFPCM-M06-2X/DEF2-TZVPPD

GEOMETRIES, FREQUENCIES, ZPE, THERMAL CORRECTIONS: IEFPCM- $\omega$ B97XD/6-311+G(D,P)

\begin{tabular}{r|cc|ccc|ccc}
\hline CPD \# & R-Group & Solvent & $\begin{array}{c}\text { Minimum } \\
\text { Energy (Eh) }\end{array}$ & $\begin{array}{c}\text { TS } \\
\text { Energy (Eh) }\end{array}$ & $\begin{array}{c}\text { E Barrier } \\
\text { (kcal/mol) }\end{array}$ & Temp C & $\begin{array}{c}\text { Free Energy } \\
\text { Correction }^{1}\end{array}$ & Barrier $^{2}$ \\
\hline $\mathbf{1}$ & CF2H & acn & -523.13217 & -523.08215 & 31.39 & 45 & -1.03 & 30.35 \\
$\mathbf{1}$ & CF2H & dmso & -523.13225 & -523.08231 & 31.34 & 45 & -1.04 & 30.30 \\
$\mathbf{1}$ & CF2H & mtbe & -523.13012 & -523.07825 & 32.55 & 45 & -1.05 & 31.49 \\
$\mathbf{1}$ & CF2H & thf & -523.13097 & -523.07986 & 32.08 & 45 & -1.03 & 31.04 \\
$\mathbf{1}$ & CF2H & toluene & -523.12843 & -523.07529 & 33.35 & 45 & -1.05 & 32.30 \\
$\mathbf{1}$ & CF2H & none & -523.12497 & -523.06973 & 34.67 & 45 & -1.10 & 33.57 \\
& & & & & & & & \\
$\mathbf{1}$ & CF2H & ACN & -523.13217 & -523.08215 & 31.39 & 30 & -1.04 & 30.35 \\
$\mathbf{7}$ & Benzothiazole & ACN & -1006.82140 & -1006.76979 & 32.39 & 30 & -1.75 & 30.64 \\
$\mathbf{8}$ & OPiv & ACN & -670.44132 & -670.38860 & 33.08 & 30 & -0.49 & 32.59 \\
$\mathbf{9}$ & Methyl & ACN & -324.62615 & -324.57084 & 34.71 & 70 & -1.19 & 33.52 \\
$\mathbf{1 0}$ & Phenyl & ACN & -516.35946 & -516.30348 & 35.13 & 70 & -1.00 & 34.13 \\
$\mathbf{1 1}$ & p-CN & ACN & -608.61252 & -608.55835 & 33.99 & 70 & -0.87 & \\
$\mathbf{1 2}$ & p-OCH3 & ACN & -630.88954 & -630.83273 & 35.65 & 70 & -1.01 & 33.12 \\
$\mathbf{1 3}$ & p-Cl & ACN & -975.96322 & -975.90771 & 34.83 & 70 & -0.93 & 34.63
\end{tabular}

${ }^{1}$ Free energy thermal correction from $\omega \mathrm{B} 97 \mathrm{xD} / 6-311+\mathrm{G}(\mathrm{d}, \mathrm{p})$ frequency calculation 
ENERGIES: IEFPCM-M08-HX/6-311+G(D,P)

GEOMETRIES, FREQUENCIES, ZPE, THERMAL CORRECTIONS: IEFPCM- $\omega$ B97XD/6-311+G(D,P)

\begin{tabular}{r|ccccc|ccc}
\hline CPD \# & R-Group & Solvent & $\begin{array}{c}\text { Minimum } \\
\text { Energy (Eh) }\end{array}$ & $\begin{array}{c}\text { TS } \\
\text { Energy (Eh) }\end{array}$ & $\begin{array}{c}\text { E Barrier } \\
\text { (kcal/mol) }\end{array}$ & Temp C & $\begin{array}{c}\text { Free Energy } \\
\text { Correction }\end{array}$ & Barrier $^{\mathbf{1}}$ \\
\hline $\mathbf{1}$ & CF2H & acn & -523.07482 & -523.02490 & 31.33 & 45 & -1.03 & 30.29 \\
$\mathbf{1}$ & CF2H & dmso & -523.07491 & -523.02506 & 31.28 & 45 & -1.04 & 30.24 \\
$\mathbf{1}$ & CF2H & mtbe & -523.07249 & -523.02081 & 32.43 & 45 & -1.05 & 31.38 \\
$\mathbf{1}$ & CF2H & thf & -523.07347 & -523.02249 & 31.99 & 45 & -1.03 & 30.96 \\
$\mathbf{1}$ & CF2H & toluene & -523.07057 & -523.01769 & 33.18 & 45 & -1.05 & 32.13 \\
$\mathbf{1}$ & CF2H & none & -523.06660 & -523.01182 & 34.38 & 45 & -1.10 & 33.28 \\
& & & & & & & & \\
$\mathbf{1}$ & CF2H & ACN & -523.07482 & -523.02490 & 31.33 & 30 & -1.04 & 30.29 \\
$\mathbf{7}$ & Benzothizole & ACN & -1006.79219 & -1006.74073 & 32.29 & 30 & -1.75 & 30.54 \\
$\mathbf{8}$ & OPiv & ACN & -670.42784 & -670.37509 & 33.10 & 30 & -0.49 & 32.60 \\
$\mathbf{9}$ & Methyl & ACN & -324.61935 & -324.56380 & 34.86 & 70 & -1.19 & 33.67 \\
$\mathbf{1 0}$ & Phenyl & ACN & -516.35214 & -516.29629 & 35.05 & 70 & -1.00 & 34.05 \\
$\mathbf{1 1}$ & p-CN & ACN & -608.59073 & -608.53659 & 33.98 & 70 & -0.87 & 33.11 \\
$\mathbf{1 2}$ & p-OCH3 & ACN & -630.87023 & -630.81370 & 35.47 & 70 & -1.01 & 34.46 \\
$\mathbf{1 3}$ & p-Cl & ACN & -975.94448 & -975.88916 & 34.71 & 70 & -0.93 &
\end{tabular}

${ }^{1}$ Free energy thermal correction from $\omega \mathrm{B} 97 \mathrm{xD} / 6-311+\mathrm{G}(\mathrm{d}, \mathrm{p})$ frequency calculation 
ENERGIES: IEFPCM-M08-HX/DEF2-TZVPPD

GEOMETRIES, FREQUENCIES, ZPE, THERMAL CORRECTIONS: IEFPCM- $\omega$ B97XD/6-311+G(D,P)

\begin{tabular}{|c|c|c|c|c|c|c|c|c|}
\hline CPD \# & R-Group & Solvent & $\begin{array}{l}\text { Minimum } \\
\text { Energy (Eh) }\end{array}$ & $\begin{array}{c}\text { TS } \\
\text { Energy (Eh) }\end{array}$ & $\begin{array}{c}\text { E Barrier } \\
\text { (kcal/mol) }\end{array}$ & Temp C & $\begin{array}{l}\text { Free Energ } \\
\text { Correction }^{1}\end{array}$ & Barrier \\
\hline 1 & $\mathrm{CF} 2 \mathrm{H}$ & $\mathrm{acn}$ & -523.14813 & -523.09851 & 31.14 & 45 & -1.03 & 29.97 \\
\hline 1 & $\mathrm{CF} 2 \mathrm{H}$ & dmso & -523.14845 & -523.09911 & 30.96 & 45 & -1.04 & 29.92 \\
\hline 1 & $\mathrm{CF} 2 \mathrm{H}$ & mtbe & -523.14637 & -523.09531 & 32.04 & 45 & -1.05 & 30.99 \\
\hline 1 & $\mathrm{CF} 2 \mathrm{H}$ & thf & -523.14721 & -523.09681 & 31.63 & 45 & -1.03 & 30.60 \\
\hline 1 & $\mathrm{CF} 2 \mathrm{H}$ & toluene & -523.14473 & -523.09255 & 32.75 & 45 & -1.05 & 31.70 \\
\hline 1 & $\mathrm{CF} 2 \mathrm{H}$ & none & -523.14135 & -523.08738 & 33.87 & 45 & -1.10 & 32.77 \\
\hline 1 & $\mathrm{CF} 2 \mathrm{H}$ & $\mathrm{ACN}$ & -523.14837 & -523.09896 & 31.00 & 30 & -1.04 & 29.97 \\
\hline 7 & Benzothiazole & $\mathrm{ACN}$ & -1006.88832 & -1006.83747 & 31.91 & 30 & -1.75 & 30.15 \\
\hline 8 & OPiv & $\mathrm{ACN}$ & -670.52253 & -670.47059 & 32.60 & 30 & -0.49 & 32.10 \\
\hline 9 & Methyl & $\mathrm{ACN}$ & -324.66751 & -324.61281 & 34.33 & 70 & -1.19 & 33.14 \\
\hline 10 & Phenyl & $\mathrm{ACN}$ & -516.42961 & -516.37425 & 34.74 & 70 & -1.00 & 33.74 \\
\hline 11 & $\mathrm{p}-\mathrm{CN}$ & $\mathrm{ACN}$ & -608.68053 & -608.62684 & 33.69 & 70 & -0.87 & 32.82 \\
\hline 12 & $\mathrm{p}-\mathrm{OCH} 3$ & $\mathrm{ACN}$ & -630.96384 & -630.90770 & 35.23 & 70 & -1.01 & 34.22 \\
\hline 13 & $\mathrm{p}-\mathrm{Cl}$ & $\mathrm{ACN}$ & -976.02649 & -975.97154 & 34.48 & 70 & -0.93 & 33.55 \\
\hline
\end{tabular}

${ }^{1}$ Free energy thermal correction from $\omega \mathrm{B} 97 \mathrm{xD} / 6-311+\mathrm{G}(\mathrm{d}, \mathrm{p})$ frequency calculation 


\begin{tabular}{|c|c|c|c|c|c|c|c|c|}
\hline Energies & $\begin{array}{l}\text { B3LYP } \\
6-311+G(d, p)\end{array}$ & $\begin{array}{l}\omega B 97 x D \\
6-311+G(d, p)\end{array}$ & $\begin{array}{l}\omega B 97 x D \\
\text { def2-TZVPPD }\end{array}$ & $\begin{array}{l}M 06-2 X \\
6-311+G(d, p)\end{array}$ & $\begin{array}{l}\text { M06-2X } \\
\text { def2-TZVPPD }\end{array}$ & $\begin{array}{l}M 08-H X \\
6-311+G(d, p)\end{array}$ & $\begin{array}{l}\text { M08-HX } \\
\text { def2-TZVPPD }\end{array}$ & Experiment \\
\hline $\mathrm{CF}_{2} \mathrm{H}$ & 24.35 & 29.91 & 29.88 & 31.91 & 31.39 & 31.33 & 31.00 & 25.6 \\
\hline Benzothiazole & 25.89 & 30.76 & 30.70 & 32.90 & 32.39 & 32.29 & 31.91 & 26.4 \\
\hline O-Pivolate & 25.48 & 31.29 & 31.14 & 33.70 & 33.08 & 33.10 & 32.60 & 27.8 \\
\hline Cyanophenyl & 26.95 & 32.02 & 32.03 & 34.41 & 33.99 & 33.98 & 33.69 & 28.4 \\
\hline Chlorophenyl & 27.62 & 32.65 & 32.80 & 35.19 & 34.83 & 34.71 & 34.48 & 29.0 \\
\hline Methyl & 27.28 & 32.74 & 32.57 & 35.35 & 34.71 & 34.86 & 34.33 & 29.5 \\
\hline Phenyl & 28.04 & 33.12 & 33.10 & 35.56 & 35.13 & 35.05 & 34.74 & 29.5 \\
\hline Methoxyphenyl & 28.62 & 33.63 & 33.67 & 36.02 & 35.65 & 35.47 & 35.23 & 29.8 \\
\hline$R^{2}$ & 0.860 & 0.963 & 0.941 & 0.985 & 0.969 & 0.985 & 0.967 & 1.000 \\
\hline slope & 1.002 & 1.195 & 1.152 & 1.068 & 1.028 & 1.042 & 1.013 & 1.000 \\
\hline intercept & 1.412 & -10.011 & -8.587 & -8.469 & -6.608 & -7.025 & -5.681 & 0.000 \\
\hline$M S E$ & 1.473 & -3.764 & -3.737 & -6.129 & -5.646 & -5.598 & -5.247 & 0.000 \\
\hline
\end{tabular}




\begin{tabular}{|c|c|c|c|c|c|c|c|c|c|}
\hline Free Energies & $\begin{array}{l}\text { B3LYP } \\
6-311+G(d, p)^{I}\end{array}$ & $\begin{array}{l}\omega B 97 x D \\
6-311+G(d, p)^{1}\end{array}$ & 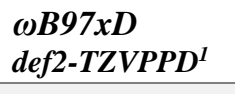 & $\begin{array}{l}M 06-2 X \\
6-311+G(d, p)^{I}\end{array}$ & $\begin{array}{l}\text { M06-2X } \\
\text { def2-TZVPPD }\end{array}$ & $\begin{array}{l}M 08-H X \\
6-311+G(d, p)^{1}\end{array}$ & $\begin{array}{l}\text { M08-HX } \\
\text { def2-TZVPPD }\end{array}$ & Experiment & MSE Shifted \\
\hline $\mathrm{CF}_{2} \mathrm{H}^{2}$ & 23.27 & 28.87 & 28.84 & 30.87 & 30.35 & 30.29 & 29.97 & 25.6 & 26.14 \\
\hline Benzothiazole $^{2}$ & 24.36 & 29.01 & 28.95 & 31.14 & 30.64 & 30.54 & 30.15 & 26.4 & 26.28 \\
\hline O-Pivolate ${ }^{2}$ & 24.77 & 30.79 & 30.65 & 33.21 & 32.59 & 32.60 & 32.10 & 27.8 & 28.06 \\
\hline Cyanophenyl $^{3}$ & 25.93 & 31.15 & 31.16 & 33.54 & 33.12 & 33.11 & 32.82 & 28.4 & 28.42 \\
\hline Chlorophenyl $^{3}$ & 26.57 & 31.72 & 31.87 & 34.26 & 33.90 & 33.78 & 33.55 & 29.0 & 28.99 \\
\hline Methyl ${ }^{3}$ & 26.08 & 31.56 & 31.38 & 34.16 & 33.52 & 33.67 & 33.14 & 29.5 & 28.83 \\
\hline Phenyl $^{3}$ & 26.99 & 32.12 & 32.09 & 34.56 & 34.13 & 34.05 & 33.74 & 29.5 & 29.39 \\
\hline Methoxyphenyl ${ }^{3}$ & 27.55 & 32.62 & 32.66 & 35.01 & 34.63 & 34.46 & 34.22 & 29.8 & 29.89 \\
\hline$R^{2}$ & 0.918 & 0.957 & 0.939 & 0.971 & 0.961 & 0.972 & 0.958 & 1.000 & 0.957 \\
\hline slope & 1.027 & 1.100 & 1.065 & 0.985 & 0.955 & 0.963 & 0.940 & 1.000 & 1.100 \\
\hline intercept & 1.870 & -5.831 & -4.727 & -4.588 & -3.137 & -3.343 & -2.263 & 0.000 & -2.828 \\
\hline$M S E$ & -2.560 & 2.729 & -2.702 & -5.094 & -4.610 & -4.562 & -4.211 & 0.000 & 0.000 \\
\hline
\end{tabular}

${ }^{1}$ Energies plus $\omega \mathrm{B} 97 \mathrm{xD} / 6-311+\mathrm{G}(\mathrm{d}, \mathrm{p})$ thermal corrections.

${ }^{2}$ Free energy calculations carried out at $30 \mathrm{C}$

${ }^{3}$ Free energy calculations carried out at $70 \mathrm{C}$ 


\section{Optimized XYZ Coordinates}

\begin{tabular}{lccc} 
14 & \multicolumn{4}{l}{} \\
difluoromethyl Minimum in Acetone wb97 \\
C & 0.16019 & -0.30773 & -0.04229 \\
C & -0.65397 & 0.73007 & 0.61221 \\
C & -2.02528 & 0.11276 & 0.45289 \\
H & -0.30012 & 1.25175 & 1.49004 \\
H & -2.79094 & 0.07114 & 1.21413 \\
C & -1.69442 & 1.34106 & -0.31565 \\
H & -2.15254 & 2.25708 & 0.03265 \\
H & -1.53432 & 1.26558 & -1.38442 \\
C & 1.64339 & -0.31308 & -0.19214 \\
H & 2.01532 & -1.19493 & -0.71249 \\
F & 2.22620 & -0.24543 & 1.04237 \\
F & 2.04536 & 0.80395 & -0.86781 \\
N & -0.50572 & -1.29241 & -0.51550 \\
O & -1.84010 & -1.12611 & -0.21158
\end{tabular}

14

difluoromethyl Minimum in Acetonitrile wb97xd/6-311+G(d,p)

$\begin{array}{lrrr}\mathrm{C} & 0.16018 & -0.30772 & -0.04249 \\ \mathrm{C} & -0.65391 & 0.72986 & 0.61232 \\ \mathrm{C} & -2.02530 & 0.11276 & 0.45309 \\ \mathrm{H} & -0.30013 & 1.25135 & 1.49027 \\ \mathrm{H} & -2.79068 & 0.07104 & 1.21459 \\ \mathrm{C} & -1.69449 & 1.34105 & -0.31535 \\ \mathrm{H} & -2.15241 & 2.25701 & 0.03330 \\ \mathrm{H} & -1.53448 & 1.26574 & -1.38416 \\ \mathrm{C} & 1.64341 & -0.31299 & -0.19254 \\ \mathrm{H} & 2.01554 & -1.19413 & -0.71385 \\ \mathrm{~F} & 2.22637 & -0.24646 & 1.04203 \\ \mathrm{~F} & 2.04521 & 0.80496 & -0.86699 \\ \mathrm{~N} & -0.50569 & -1.29233 & -0.51594 \\ \mathrm{O} & -1.84019 & -1.12612 & -0.21176\end{array}$

14

difluoromethyl Minimum in DMSO wb97xd/6-311+G(d,p)

$\begin{array}{lccc}\mathrm{C} & 0.16018 & -0.30771 & -0.04256 \\ \mathrm{C} & -0.65388 & 0.72979 & 0.61234 \\ \mathrm{C} & -2.02531 & 0.11276 & 0.45316 \\ \mathrm{H} & -0.30013 & 1.25124 & 1.49033 \\ \mathrm{H} & -2.79057 & 0.07100 & 1.21476 \\ \mathrm{C} & -1.69452 & 1.34104 & -0.31526 \\ \mathrm{H} & -2.15239 & 2.25698 & 0.03352 \\ \mathrm{H} & -1.53455 & 1.26578 & -1.38407 \\ \mathrm{C} & 1.64341 & -0.31295 & -0.19267 \\ \mathrm{H} & 2.01561 & -1.19386 & -0.71431 \\ \mathrm{~F} & 2.22642 & -0.24681 & 1.04191 \\ \mathrm{~F} & 2.04516 & 0.80529 & -0.86671 \\ \mathrm{~N} & -0.50567 & -1.29230 & -0.51609 \\ \mathrm{O} & -1.84022 & -1.12612 & -0.21182\end{array}$


14

difluoromethyl Minimum in MTBE wb97xd/6-311+G(d,p)

$\begin{array}{lccc}\mathrm{C} & 0.16026 & -0.30804 & -0.04053 \\ \mathrm{C} & -0.65444 & 0.73161 & 0.61143 \\ \mathrm{C} & -2.02511 & 0.11296 & 0.45123 \\ \mathrm{H} & -0.30011 & 1.25464 & 1.48841 \\ \mathrm{H} & -2.79302 & 0.07259 & 1.21049 \\ \mathrm{C} & -1.69356 & 1.34120 & -0.31816 \\ \mathrm{H} & -2.15301 & 2.25782 & 0.02715 \\ \mathrm{H} & -1.53236 & 1.26429 & -1.38663 \\ \mathrm{C} & 1.64331 & -0.31392 & -0.18888 \\ \mathrm{H} & 2.01373 & -1.20130 & -0.70148 \\ \mathrm{~F} & 2.22496 & -0.23696 & 1.04505 \\ \mathrm{~F} & 2.04624 & 0.79580 & -0.87451 \\ \mathrm{~N} & -0.50602 & -1.29325 & -0.51186 \\ \mathrm{O} & -1.83958 & -1.12597 & -0.21004\end{array}$

14

difluoromethyl Minimum in THF wb97xd/6-311+G(d,p)

$\begin{array}{lccc}\mathrm{C} & 0.16018 & -0.30777 & -0.04137 \\ \mathrm{C} & -0.65417 & 0.73087 & 0.61240 \\ \mathrm{C} & -2.02543 & 0.11303 & 0.45165 \\ \mathrm{H} & -0.29994 & 1.25231 & 1.49032 \\ \mathrm{H} & -2.79277 & 0.07274 & 1.21136 \\ \mathrm{C} & -1.69321 & 1.34163 & -0.31682 \\ \mathrm{H} & -2.15222 & 2.25782 & 0.03002 \\ \mathrm{H} & -1.53170 & 1.26614 & -1.38538 \\ \mathrm{C} & 1.64325 & -0.31372 & -0.19055 \\ \mathrm{H} & 2.01417 & -1.19854 & -0.70688 \\ \mathrm{~F} & 2.22576 & -0.24133 & 1.04373 \\ \mathrm{~F} & 2.04621 & 0.79956 & -0.87150 \\ \mathrm{~N} & -0.50637 & -1.29299 & -0.51292 \\ \mathrm{O} & -1.84055 & -1.12597 & -0.21137\end{array}$

14

difluoromethyl Minimum in Toluene wb97xd/6-311+G(d,p)

$\begin{array}{lrrr}\mathrm{C} & 0.16029 & -0.30839 & -0.03873 \\ \mathrm{C} & -0.65478 & 0.73298 & 0.61122 \\ \mathrm{C} & -2.02513 & 0.11349 & 0.44928 \\ \mathrm{H} & -0.30000 & 1.25641 & 1.48793 \\ \mathrm{H} & -2.79553 & 0.07530 & 1.20630 \\ \mathrm{C} & -1.69179 & 1.34184 & -0.32063 \\ \mathrm{H} & -2.15253 & 2.25900 & 0.02185 \\ \mathrm{H} & -1.52851 & 1.26403 & -1.38870 \\ \mathrm{C} & 1.64322 & -0.31487 & -0.18579 \\ \mathrm{H} & 2.01204 & -1.20756 & -0.69086 \\ \mathrm{~F} & 2.22396 & -0.22895 & 1.04751 \\ \mathrm{~F} & 2.04718 & 0.78771 & -0.88119 \\ \mathrm{~N} & -0.50673 & -1.29433 & -0.50766 \\ \mathrm{O} & -1.83992 & -1.12576 & -0.20899\end{array}$


14

difluoromethyl Minimum in Vacuum wb97xd/6-311+G(d,p)

$\begin{array}{llrc}\mathrm{C} & 0.16050 & -0.30966 & -0.03525 \\ \mathrm{C} & -0.65517 & 0.73559 & 0.60956 \\ \mathrm{C} & -2.02445 & 0.11454 & 0.44605 \\ \mathrm{H} & -0.29949 & 1.26143 & 1.48470 \\ \mathrm{H} & -2.79883 & 0.08038 & 1.19958 \\ \mathrm{C} & -1.68899 & 1.34240 & -0.32610 \\ \mathrm{H} & -2.15182 & 2.26098 & 0.01039 \\ \mathrm{H} & -1.52265 & 1.26109 & -1.39342 \\ \mathrm{C} & 1.64338 & -0.31627 & -0.17939 \\ \mathrm{H} & 2.00937 & -1.21929 & -0.66926 \\ \mathrm{~F} & 2.22105 & -0.21226 & 1.05232 \\ \mathrm{~F} & 2.04784 & 0.77153 & -0.89400 \\ \mathrm{~N} & -0.50736 & -1.29649 & -0.50027 \\ \mathrm{O} & -1.83958 & -1.12527 & -0.20553\end{array}$

14

difluoromethyl Transition State in Acetone wb97xd/6-311+G(d,p)

$\begin{array}{lrrr}\mathrm{C} & 0.18469 & -0.07473 & -0.14899 \\ \mathrm{C} & -0.36705 & 1.12281 & 0.10637 \\ \mathrm{C} & -1.85863 & 1.25162 & 0.26754 \\ \mathrm{C} & -2.45897 & -0.09043 & 0.05572 \\ \mathrm{H} & 0.25304 & 2.00290 & 0.20061 \\ \mathrm{H} & -2.16202 & 1.61299 & 1.26047 \\ \mathrm{H} & -3.53081 & -0.25939 & 0.09791 \\ \mathrm{H} & -2.31861 & 1.95031 & -0.44493 \\ \mathrm{O} & -1.82188 & -1.14754 & -0.18696 \\ \mathrm{~N} & -0.43946 & -1.28247 & -0.31883 \\ \mathrm{C} & 1.67532 & -0.25962 & -0.26120 \\ \mathrm{H} & 1.95620 & -0.99990 & -1.01095 \\ \mathrm{~F} & 2.18655 & -0.67493 & 0.94443 \\ \mathrm{~F} & 2.30248 & 0.91414 & -0.55469\end{array}$

14

difluoromethyl Transition State in Acetonitrile wb97xd/6-311+G(d,p)

$\begin{array}{lrrr}\mathrm{C} & 0.18483 & -0.07327 & 0.14925 \\ \mathrm{C} & -0.36798 & 1.12361 & -0.10660 \\ \mathrm{C} & -1.85972 & 1.25059 & -0.26727 \\ \mathrm{C} & -2.45929 & -0.09161 & -0.05618 \\ \mathrm{H} & 0.25086 & 2.00454 & -0.20086 \\ \mathrm{H} & -2.32059 & 1.94824 & 0.44555 \\ \mathrm{H} & -3.53106 & -0.26087 & -0.09894 \\ \mathrm{H} & -2.16407 & 1.61181 & -1.25988 \\ \mathrm{O} & -1.82171 & -1.14816 & 0.18642 \\ \mathrm{~N} & -0.43809 & -1.28181 & 0.31895 \\ \mathrm{C} & 1.67546 & -0.25648 & 0.26393 \\ \mathrm{H} & 1.95673 & -0.98874 & 1.02134 \\ \mathrm{~F} & 2.30219 & 0.92050 & 0.54510 \\ \mathrm{~F} & 2.18766 & -0.68430 & -0.93732\end{array}$


14

difluoromethyl Transition State in DMSO wb97xd/6-311+G(d,p)

$\begin{array}{lrrr}\mathrm{C} & 0.18491 & -0.07269 & -0.14976 \\ \mathrm{C} & -0.36821 & 1.12400 & 0.10622 \\ \mathrm{C} & -1.85995 & 1.25030 & 0.26726 \\ \mathrm{C} & -2.45935 & -0.09191 & 0.05643 \\ \mathrm{H} & 0.25019 & 2.00525 & 0.20020 \\ \mathrm{H} & -2.16434 & 1.61142 & 1.25988 \\ \mathrm{H} & -3.53111 & -0.26121 & 0.09954 \\ \mathrm{H} & -2.32138 & 1.94768 & -0.44542 \\ \mathrm{O} & -1.82168 & -1.14829 & -0.18628 \\ \mathrm{~N} & -0.43767 & -1.28152 & -0.31918 \\ \mathrm{C} & 1.67556 & -0.25552 & -0.26478 \\ \mathrm{H} & 1.95727 & -0.98542 & -1.02429 \\ \mathrm{~F} & 2.18719 & -0.68724 & 0.93549 \\ \mathrm{~F} & 2.30267 & 0.92214 & -0.54189\end{array}$

14

difluoromethyl Transition State in MTBE wb97xd/6-311+G(d,p)

$\begin{array}{lrrr}\text { C } & 0.18362 & -0.08354 & -0.14436 \\ \mathrm{C} & -0.36291 & 1.11746 & 0.10750 \\ \mathrm{C} & -1.85418 & 1.25804 & 0.26480 \\ \mathrm{C} & -2.45700 & -0.08511 & 0.05374 \\ \mathrm{H} & 0.26458 & 1.99229 & 0.20406 \\ \mathrm{H} & -2.15521 & 1.62572 & 1.25665 \\ \mathrm{H} & -3.52879 & -0.25466 & 0.09441 \\ \mathrm{H} & -2.30557 & 1.95934 & -0.45138 \\ \mathrm{O} & -1.82111 & -1.14547 & -0.18764 \\ \mathrm{~N} & -0.44726 & -1.28660 & -0.31619 \\ \mathrm{C} & 1.67442 & -0.27614 & -0.24492 \\ \mathrm{H} & 1.95064 & -1.05923 & -0.95198 \\ \mathrm{~F} & 2.18518 & -0.62055 & 0.98005 \\ \mathrm{~F} & 2.30041 & 0.87859 & -0.60870\end{array}$

14

difluoromethyl Transition State in THF wb97xd/6-311+G(d,p)

$\begin{array}{lrrr}\text { C } & 0.18411 & -0.07936 & -0.14685 \\ \text { C } & -0.36479 & 1.12000 & 0.10691 \\ \text { C } & -1.85616 & 1.25497 & 0.26668 \\ \text { C } & -2.45787 & -0.08757 & 0.05469 \\ \text { H } & 0.25927 & 1.99727 & 0.20245 \\ \text { H } & -2.15792 & 1.61907 & 1.25939 \\ \text { H } & -3.52970 & -0.25680 & 0.09600 \\ \text { H } & -2.31190 & 1.95543 & -0.44719 \\ \text { O } & -1.82148 & -1.14630 & -0.18770 \\ \text { N } & -0.44361 & -1.28457 & -0.31793 \\ \text { C } & 1.67482 & -0.26851 & -0.25271 \\ \text { H } & 1.95337 & -1.03163 & -0.98024 \\ \text { F } & 2.18547 & -0.64664 & 0.96372 \\ \text { F } & 2.30156 & 0.89574 & -0.58323\end{array}$


14

difluoromethyl Transition State in Toluene wb97xd/6-311+G(d,p)

$\begin{array}{lrrr}\text { C } & 0.18287 & -0.08998 & -0.13971 \\ \text { C } & -0.36058 & 1.11346 & 0.10827 \\ \text { C } & -1.85174 & 1.26313 & 0.26000 \\ \text { C } & -2.45539 & -0.08190 & 0.05251 \\ \text { H } & 0.27224 & 1.98454 & 0.20597 \\ \text { H } & -2.15226 & 1.63873 & 1.24948 \\ \text { H } & -3.52690 & -0.25309 & 0.09268 \\ \text { H } & -2.29531 & 1.96463 & -0.46164 \\ \text { O } & -1.81939 & -1.14496 & -0.18634 \\ \text { N } & -0.45281 & -1.28981 & -0.31205 \\ \text { C } & 1.67397 & -0.28702 & -0.23252 \\ \text { H } & 1.94604 & -1.09937 & -0.90776 \\ \text { F } & 2.18534 & -0.57974 & 1.00306 \\ \text { F } & 2.29758 & 0.85161 & -0.64693\end{array}$

14

difluoromethyl Transition State in Vacuum wb97xd/6-311+G(d,p) $E=-523.034072$ hartree

$\begin{array}{lrrr}\mathrm{C} & 0.18207 & -0.09731 & -0.13331 \\ \mathrm{C} & -0.35940 & 1.10868 & 0.10858 \\ \mathrm{C} & -1.85035 & 1.26997 & 0.25208 \\ \mathrm{C} & -2.45230 & -0.07960 & 0.05135 \\ \mathrm{H} & 0.28026 & 1.97519 & 0.20592 \\ \mathrm{H} & -2.14971 & 1.65882 & 1.23772 \\ \mathrm{H} & -3.52276 & -0.25668 & 0.09108 \\ \mathrm{H} & -2.28255 & 1.97127 & -0.47814 \\ \mathrm{O} & -1.81321 & -1.14625 & -0.18363 \\ \mathrm{~N} & -0.45922 & -1.29320 & -0.30512 \\ \mathrm{C} & 1.67422 & -0.29774 & -0.21703 \\ \mathrm{H} & 1.93959 & -1.14207 & -0.85555 \\ \mathrm{~F} & 2.18464 & -0.53181 & 1.02662 \\ \mathrm{~F} & 2.29202 & 0.81981 & -0.68952\end{array}$

14

difluoromethyl Triplet Minimum in Vacuum wb97xd/6-311+G(d,p) $E=-523.017864$ hartree

$\begin{array}{lrrr}\mathrm{C} & 0.18945 & -0.16103 & -0.03723 \\ \mathrm{C} & -0.33587 & 1.12304 & -0.06384 \\ \mathrm{C} & -1.80861 & 1.28120 & -0.04322 \\ \mathrm{C} & -2.45975 & -0.01192 & 0.27659 \\ \mathrm{H} & 0.32050 & 1.98188 & -0.11492 \\ \mathrm{H} & -2.11221 & 2.04683 & 0.68016 \\ \mathrm{H} & -3.53167 & -0.13523 & 0.34257 \\ \mathrm{H} & -2.14240 & 1.66355 & -1.02760 \\ \mathrm{O} & -1.86749 & -1.18157 & -0.14472 \\ \mathrm{~N} & -0.49393 & -1.29093 & -0.08325 \\ \mathrm{C} & 1.67612 & -0.39686 & 0.00931 \\ \mathrm{H} & 1.92295 & -1.45832 & -0.02978 \\ \mathrm{~F} & 2.20156 & 0.13708 & 1.14850 \\ \mathrm{~F} & 2.28424 & 0.23890 & -1.03289\end{array}$


14

Difluoromethyl Recant in Vacuum CAS(4,4)/6-31+G(d) E= -520.396015 hartree

$\begin{array}{lrrr}\mathrm{C} & 0.16694 & -0.30909 & -0.02307 \\ \mathrm{C} & -0.66085 & 0.75242 & 0.59841 \\ \mathrm{C} & -2.03264 & 0.10861 & 0.43722 \\ \mathrm{H} & -0.31417 & 1.28680 & 1.46256 \\ \mathrm{H} & -2.81094 & 0.07784 & 1.17425 \\ \mathrm{C} & -1.69860 & 1.32805 & -0.33961 \\ \mathrm{H} & -2.16620 & 2.24346 & -0.02288 \\ \mathrm{H} & -1.53795 & 1.24226 & -1.39969 \\ \mathrm{C} & 1.65184 & -0.31537 & -0.16727 \\ \mathrm{H} & 2.02037 & -1.21840 & -0.62160 \\ \mathrm{~F} & 2.22203 & -0.17165 & 1.04387 \\ \mathrm{~F} & 2.04574 & 0.73657 & -0.90710 \\ \mathrm{~N} & -0.50209 & -1.30529 & -0.46545 \\ \mathrm{O} & -1.83080 & -1.12088 & -0.19993\end{array}$

14

difluoromethyl Transition State in Vacuum CAS(4,4,uno)/6-31+G(d) E= -520.344130 hartree

$\begin{array}{lrrr}\mathrm{C} & 0.19530 & -0.06294 & -0.14523 \\ \mathrm{C} & -0.38401 & 1.13002 & 0.10566 \\ \mathrm{C} & -1.87743 & 1.25894 & 0.25521 \\ \mathrm{C} & -2.47575 & -0.10145 & 0.06203 \\ \mathrm{H} & 0.22700 & 2.00651 & 0.20567 \\ \mathrm{H} & -2.13250 & 1.65934 & 1.23521 \\ \mathrm{H} & -3.51992 & -0.32362 & 0.11439 \\ \mathrm{H} & -2.27985 & 1.96470 & -0.46978 \\ \mathrm{O} & -1.76383 & -1.17879 & -0.18710 \\ \mathrm{~N} & -0.46850 & -1.26276 & -0.30737 \\ \mathrm{C} & 1.68349 & -0.25008 & -0.25752 \\ \mathrm{H} & 1.95341 & -0.95959 & -1.02102 \\ \mathrm{~F} & 2.18628 & -0.68876 & 0.91168 \\ \mathrm{~F} & 2.29065 & 0.91936 & -0.52690\end{array}$


23

Benzothiophene Minimum in Acetonitrile wb97xd/6-311+G(d,p)

$\begin{array}{lrrr}\mathrm{C} & 4.00905 & 0.45502 & -0.30823 \\ \mathrm{H} & 4.81665 & 0.69298 & -0.98538 \\ \mathrm{C} & 3.61970 & 1.31455 & 0.84212 \\ \mathrm{H} & 4.08407 & 2.29080 & 0.88357 \\ \mathrm{H} & 3.40407 & 0.84936 & 1.79663 \\ \mathrm{C} & 2.64213 & 1.09584 & -0.30240 \\ \mathrm{H} & 2.32356 & 1.90643 & -0.94141 \\ \mathrm{C} & 1.80085 & -0.10107 & -0.12038 \\ \mathrm{~N} & 2.46110 & -1.19767 & -0.00849 \\ \mathrm{O} & 3.80678 & -0.94056 & -0.15901 \\ \mathrm{C} & -1.70088 & 0.71202 & -0.06868 \\ \mathrm{~N} & -0.34666 & 0.98809 & -0.12878 \\ \mathrm{C} & 0.34425 & -0.10225 & -0.07035 \\ \mathrm{~S} & -0.56481 & -1.59711 & 0.06382 \\ \mathrm{C} & -2.02630 & -0.65097 & 0.04195 \\ \mathrm{C} & -3.35146 & -1.07945 & 0.11656 \\ \mathrm{H} & -3.59741 & -2.13070 & 0.20239 \\ \mathrm{C} & -4.34586 & -0.11687 & 0.07601 \\ \mathrm{H} & -5.38380 & -0.42354 & 0.13120 \\ \mathrm{C} & -4.03298 & 1.24685 & -0.03525 \\ \mathrm{H} & -4.83427 & 1.97576 & -0.06421 \\ \mathrm{C} & -2.71938 & 1.67031 & -0.10771 \\ \mathrm{H} & -2.46586 & 2.72029 & -0.19284\end{array}$

23

Benzothiophene Transition State in Acetonitrile wb97xd/6-311+G(d,p)

$\begin{array}{llll}\text { C } & -4.43849 & -0.02883 & -0.00003\end{array}$

$\begin{array}{llll}\mathrm{H} & -5.49860 & -0.26287 & -0.00005\end{array}$

$\begin{array}{llll}\text { C } & -3.92996 & 1.36575 & 0.00003\end{array}$

$\begin{array}{llll}\mathrm{H} & -4.34881 & 1.88678 & 0.87260\end{array}$

$\begin{array}{llll}\mathrm{H} & -4.34881 & 1.88685 & -0.87250\end{array}$

$\begin{array}{llll}\text { C } & -2.42667 & 1.31292 & 0.00003\end{array}$

$\begin{array}{llll}\mathrm{H} & -1.86061 & 2.23353 & 0.00006\end{array}$

$\begin{array}{llll}\mathrm{C} & -1.78113 & 0.13093 & -0.00001\end{array}$

$\begin{array}{llll}\mathrm{N} & -2.33709 & -1.12731 & -0.00005\end{array}$

$\begin{array}{llll}\text { O } & -3.72429 & -1.06507 & -0.00006\end{array}$

$\begin{array}{llll}\text { C } & 1.79903 & 0.73454 & 0.00000\end{array}$

$\begin{array}{llll}\mathrm{N} & 0.46700 & 1.10634 & -0.00001\end{array}$

$\begin{array}{llll}\text { C } & -0.30428 & 0.07171 & -0.00000\end{array}$

$\begin{array}{llll}\mathrm{S} & 0.50593 & -1.49048 & 0.00001\end{array}$

$\begin{array}{llll}\mathrm{C} & 2.03076 & -0.65142 & 0.00001\end{array}$

$\begin{array}{llll}\text { C } & 3.32413 & -1.17361 & 0.00002\end{array}$

$\begin{array}{llll}\mathrm{H} & 3.49630 & -2.24306 & 0.00003\end{array}$

$\begin{array}{llll}\text { C } & 4.38453 & -0.28285 & 0.00002\end{array}$

$\begin{array}{llll}\mathrm{H} & 5.39920 & -0.66368 & 0.00003\end{array}$

$\begin{array}{llll}\mathrm{C} & 4.16523 & 1.10320 & 0.00001\end{array}$

$\begin{array}{llll}\mathrm{H} & 5.01460 & 1.77636 & 0.00001\end{array}$

$\begin{array}{llll}\mathrm{C} & 2.88251 & 1.61978 & 0.00000\end{array}$

$\begin{array}{llll}\mathrm{H} & 2.70294 & 2.68851 & -0.00001\end{array}$ 
29

O-Pivolate Minimum in Acetonitrile wb97xd/6-311+G(d,p)

$\begin{array}{llll}\text { C } & 3.68235 & 0.69099 & -0.33498\end{array}$

$\begin{array}{llll}\mathrm{H} & 4.55389 & 0.91220 & -0.93468\end{array}$

$\begin{array}{llll}\mathrm{C} & 2.65143 & 1.70721 & 0.01014\end{array}$

$\begin{array}{llll}\mathrm{H} & 2.72870 & 2.65663 & -0.50300\end{array}$

$\begin{array}{llll}\mathrm{H} & 2.26612 & 1.74323 & 1.02223\end{array}$

$\begin{array}{llll}\text { C } & 2.27672 & 0.51375 & -0.85664\end{array}$

$\begin{array}{llll}\mathrm{H} & 1.96527 & 0.63868 & -1.88467\end{array}$

$\begin{array}{llll}\text { C } & 1.85405 & -0.66103 & -0.07258\end{array}$

$\begin{array}{llll}\text { C } & 0.54180 & -1.35086 & -0.19667\end{array}$

$\mathrm{H} \quad 0.41153 \quad-1.73776 \quad-1.21073$

$\begin{array}{llll}\mathrm{O} & -0.46714 & -0.37071 & 0.07284\end{array}$

$\begin{array}{llll}\mathrm{H} & 0.46810 & -2.17743 & 0.51124\end{array}$

$\begin{array}{llll}\mathrm{N} & 2.73730 & -1.09016 & 0.74946\end{array}$

$\begin{array}{llll}\mathrm{O} & 3.90287 & -0.34417 & 0.60286\end{array}$

$\begin{array}{llll}\text { C } & -1.73548 & -0.71962 & -0.18798\end{array}$

$\begin{array}{llll}\text { C } & -2.70446 & 0.41535 & 0.12207\end{array}$

$\begin{array}{llll}\text { C } & -2.60279 & 0.75419 & 1.61966\end{array}$

$\begin{array}{llll}\mathrm{H} & -1.60054 & 1.09725 & 1.88248\end{array}$

$\begin{array}{llll}\mathrm{H} & -2.84802 & -0.11325 & 2.23881\end{array}$

$\begin{array}{llll}\mathrm{H} & -3.31322 & 1.55078 & 1.85607\end{array}$

$\begin{array}{llll}\text { C } & -4.12756 & -0.02852 & -0.22022\end{array}$

$\begin{array}{llll}\mathrm{H} & -4.82207 & 0.78623 & 0.00033\end{array}$

$\begin{array}{llll}\mathrm{H} & -4.42404 & -0.90044 & 0.36698\end{array}$

$\begin{array}{llll}\mathrm{H} & -4.22123 & -0.28206 & -1.27859\end{array}$

$\begin{array}{llll}\text { C } & -2.31630 & 1.64352 & -0.71935\end{array}$

$\begin{array}{llll}\mathrm{H} & -1.31130 & 1.99169 & -0.47438\end{array}$

$\begin{array}{llll}\mathrm{H} & -3.02150 & 2.45409 & -0.51742\end{array}$

$\begin{array}{llll}\mathrm{H} & -2.35611 & 1.41857 & -1.78887\end{array}$

$\begin{array}{llll}\mathrm{O} & -2.03014 & -1.80979 & -0.61478\end{array}$ 
29

O-Pivolate Transition State in Acetonitrile wb97xd/6-311+G(d,p)

$\begin{array}{llll}\text { C } & -4.10914 & 0.78412 & 0.06394\end{array}$

$\begin{array}{llll}\mathrm{H} & -5.01129 & 1.38774 & 0.02573\end{array}$

$\begin{array}{llll}\text { C } & -3.86437 & -0.20749 & 1.14131\end{array}$

$\begin{array}{llll}\mathrm{H} & -4.71679 & -0.90078 & 1.17054\end{array}$

$\begin{array}{llll}\mathrm{H} & -3.89168 & 0.31889 & 2.10669\end{array}$

$\begin{array}{llll}\text { C } & -2.54689 & -0.87902 & 0.85622\end{array}$

$\mathrm{H} \quad-2.20078 \quad-1.65656 \quad 1.52456$

$\begin{array}{llll}\text { C } & -1.79753 & -0.51819 & -0.20309\end{array}$

$\begin{array}{llll}\text { C } & -0.47151 & -1.16778 & -0.47107\end{array}$

$\begin{array}{llll}\mathrm{H} & -0.37680 & -2.09709 & 0.09205\end{array}$

$\begin{array}{llll}\mathrm{O} & 0.55703 & -0.25226 & -0.05343\end{array}$

$\begin{array}{llll}\mathrm{H} & -0.34672 & -1.38104 & -1.53447\end{array}$

$\begin{array}{llll}\mathrm{N} & -2.09062 & 0.43076 & -1.14879\end{array}$

$\begin{array}{llll}\mathrm{O} & -3.32955 & 1.03264 & -0.89053\end{array}$

$\begin{array}{llll}\text { C } & 1.82082 & -0.62902 & -0.26648\end{array}$

$\begin{array}{llll}\mathrm{C} & 2.81445 & 0.42491 & 0.21347\end{array}$

$\begin{array}{llll}\text { C } & 2.54913 & 1.73744 & -0.54370\end{array}$

$\mathrm{H} \quad 1.54453 \quad 2.11460 \quad-0.34464$

$\begin{array}{llll}\mathrm{H} & 2.66158 & 1.60045 & -1.62293\end{array}$

$\begin{array}{llll}\mathrm{H} & 3.27194 & 2.49163 & -0.22088\end{array}$

$\begin{array}{llll}\text { C } & 4.23877 & -0.05997 & -0.06164\end{array}$

$\begin{array}{llll}\mathrm{H} & 4.40268 & -0.22594 & -1.12873\end{array}$

$\begin{array}{llll}\mathrm{H} & 4.44971 & -0.99361 & 0.46466\end{array}$

$\begin{array}{llll}\mathrm{H} & 4.95012 & 0.69588 & 0.28152\end{array}$

$\begin{array}{llll}\text { C } & 2.61465 & 0.64284 & 1.72321\end{array}$

$\begin{array}{llll}\mathrm{H} & 2.77285 & -0.28475 & 2.28072\end{array}$

$\mathrm{H} \quad 1.61133 \quad 1.01275 \quad 1.94162$

$\begin{array}{llll}\mathrm{H} & 3.33946 & 1.37977 & 2.07956\end{array}$

$\begin{array}{llll}\text { O } & 2.10801 & -1.68592 & -0.77949\end{array}$

14

Methyl Minimum in Acetonitrile wb97xd/6-311+G(d,p)

$\begin{array}{lrrr}\mathrm{C} & -1.41216 & 0.04101 & -0.38061 \\ \mathrm{H} & -2.21500 & 0.09724 & -1.10261 \\ \mathrm{C} & -1.29151 & -1.06214 & 0.61280 \\ \mathrm{H} & -1.96041 & -1.90227 & 0.47950 \\ \mathrm{H} & -1.03059 & -0.81947 & 1.63631 \\ \mathrm{C} & -0.22585 & -0.88224 & -0.46117 \\ \mathrm{H} & -0.06565 & -1.63583 & -1.22033 \\ \mathrm{C} & 0.86327 & 0.03123 & -0.05672 \\ \mathrm{C} & 2.30045 & -0.34730 & 0.02481 \\ \mathrm{H} & 2.63963 & -0.72116 & -0.94504 \\ \mathrm{H} & 2.43669 & -1.15174 & 0.75218 \\ \mathrm{H} & 2.91261 & 0.50717 & 0.31221 \\ \mathrm{~N} & 0.46214 & 1.21387 & 0.23023 \\ \mathrm{O} & -0.91969 & 1.30569 & 0.00520\end{array}$


14

Methyl Transition State in Acetonitrile wb97xd/6-311+G(d,p)

$\begin{array}{lrrr}\mathrm{C} & 1.75941 & -0.25930 & -0.00000 \\ \mathrm{H} & 2.80484 & -0.55508 & -0.00000 \\ \mathrm{C} & 1.33690 & 1.16203 & -0.00000 \\ \mathrm{H} & 1.79330 & 1.65401 & -0.87197 \\ \mathrm{H} & 1.79330 & 1.65401 & 0.87197 \\ \mathrm{C} & -0.16954 & 1.19463 & 0.00000 \\ \mathrm{H} & -0.66598 & 2.15643 & 0.00000 \\ \mathrm{C} & -0.90187 & 0.06183 & 0.00000 \\ \mathrm{C} & -2.40594 & 0.07793 & 0.00000 \\ \mathrm{H} & -2.79590 & -0.43591 & 0.88253 \\ \mathrm{H} & -2.79590 & -0.43591 & -0.88252 \\ \mathrm{H} & -2.77768 & 1.10314 & 0.00000 \\ \mathrm{~N} & -0.42067 & -1.22535 & 0.00000 \\ \mathrm{O} & 0.98437 & -1.24825 & 0.00000\end{array}$

21

Cyanophenyl Minimum in Acetonitrile wb97xd/6-311+G(d,p)

$\begin{array}{lrrr}\mathrm{C} & 2.98085 & 0.13343 & -0.34965 \\ \mathrm{H} & 3.80070 & 0.19289 & -1.05149 \\ \mathrm{C} & 2.65449 & 1.22355 & 0.61076 \\ \mathrm{H} & 3.18494 & 2.15631 & 0.47123 \\ \mathrm{H} & 2.40469 & 0.96604 & 1.63319 \\ \mathrm{C} & 1.66341 & 0.84994 & -0.48327 \\ \mathrm{H} & 1.41168 & 1.54242 & -1.27465 \\ \mathrm{C} & 0.72789 & -0.22444 & -0.08477 \\ \mathrm{~N} & 1.31567 & -1.31902 & 0.24591 \\ \mathrm{O} & 2.68892 & -1.18848 & 0.05375 \\ \mathrm{C} & -3.51239 & 0.18346 & 0.04392 \\ \mathrm{H} & -4.59052 & 0.29113 & 0.07814 \\ \mathrm{C} & -2.93869 & -1.08605 & 0.01316 \\ \mathrm{H} & -3.57009 & -1.96711 & 0.01984 \\ \mathrm{C} & -1.56004 & -1.22834 & -0.03579 \\ \mathrm{H} & -1.11290 & -2.21457 & -0.07269 \\ \mathrm{C} & -0.73599 & -0.09771 & -0.04761 \\ \mathrm{C} & -1.31690 & 1.17184 & -0.02144 \\ \mathrm{H} & -0.68737 & 2.05475 & -0.02672 \\ \mathrm{C} & -2.69963 & 1.31072 & 0.02524 \\ \mathrm{H} & -3.14018 & 2.30070 & 0.04841\end{array}$




\begin{tabular}{lccc}
21 & \multicolumn{4}{c}{} \\
Cyanophenyl Transition State in & Acetonitrile wb $97 \mathrm{xd} / 6-311+\mathrm{G}(\mathrm{d}, \mathrm{p})$ \\
$\mathrm{C}$ & -3.37454 & -0.18724 & -0.10498 \\
$\mathrm{H}$ & -4.42688 & -0.41119 & -0.25447 \\
$\mathrm{C}$ & -2.90913 & 1.08989 & 0.48722 \\
$\mathrm{H}$ & -3.31464 & 1.16439 & 1.50814 \\
$\mathrm{H}$ & -3.37819 & 1.91588 & -0.06527 \\
$\mathrm{C}$ & -1.40428 & 1.09885 & 0.43289 \\
$\mathrm{H}$ & -0.87970 & 1.97816 & 0.78176 \\
$\mathrm{C}$ & -0.70870 & 0.02346 & 0.00532 \\
$\mathrm{~N}$ & -1.22891 & -1.16371 & -0.45536 \\
$\mathrm{O}$ & -2.62986 & -1.13057 & -0.47385 \\
$\mathrm{C}$ & 3.58058 & 0.00777 & 0.01538 \\
$\mathrm{H}$ & 4.66475 & 0.00549 & 0.02414 \\
$\mathrm{C}$ & 2.87466 & -1.11831 & 0.42727 \\
$\mathrm{H}$ & 3.40775 & -2.00094 & 0.76290 \\
$\mathrm{C}$ & 1.48462 & -1.11667 & 0.41512 \\
$\mathrm{H}$ & 0.93657 & -1.99278 & 0.74101 \\
$\mathrm{C}$ & 0.78051 & 0.01451 & -0.00526 \\
$\mathrm{C}$ & 1.49651 & 1.13698 & -0.42659 \\
$\mathrm{H}$ & 0.96154 & 2.01229 & -0.77802 \\
$\mathrm{C}$ & 2.88670 & 1.13503 & -0.41252 \\
$\mathrm{H}$ & 3.42838 & 2.01359 & -0.74493 \\
& & & \\
22 & & & \\
$\mathrm{Phenyl}$ Minimum & in $\mathrm{Acetonitrile}$ & $\mathrm{wb} 97 \mathrm{xd} / 6-311+\mathrm{G}(\mathrm{d}, \mathrm{p})$ \\
$\mathrm{C}$ & -3.68132 & -0.20538 & -0.32536 \\
$\mathrm{H}$ & -4.50773 & -0.29140 & -1.01637 \\
$\mathrm{C}$ & -3.31448 & -1.27435 & 0.64292 \\
$\mathrm{H}$ & -3.82222 & -2.22181 & 0.51989 \\
$\mathrm{H}$ & -3.05931 & -0.99892 & 1.65926 \\
$\mathrm{C}$ & -2.34641 & -0.88918 & -0.46739 \\
$\mathrm{H}$ & -2.08705 & -1.58233 & -1.25568 \\
$\mathrm{C}$ & -1.43874 & 0.21632 & -0.09393 \\
$\mathrm{~N}$ & -2.05193 & 1.29888 & 0.22922 \\
$\mathrm{O}$ & -3.41695 & 1.12961 & 0.06030 \\
$\mathrm{C}$ & 2.80299 & -0.03877 & 0.00588 \\
$\mathrm{C}$ & 2.18751 & 1.21761 & -0.00437 \\
$\mathrm{H}$ & 2.79038 & 2.11687 & 0.01132 \\
$\mathrm{C}$ & 0.80843 & 1.30219 & -0.04405 \\
$\mathrm{H}$ & 0.32684 & 2.27152 & -0.06444 \\
$\mathrm{C}$ & 0.02858 & 0.14072 & -0.06567 \\
$\mathrm{C}$ & 0.65292 & -1.10799 & -0.05894 \\
$\mathrm{H}$ & 0.05811 & -2.01338 & -0.07123 \\
$\mathrm{C}$ & 2.03532 & -1.20352 & -0.02279 \\
$\mathrm{H}$ & 2.51631 & -2.17346 & -0.01447 \\
$\mathrm{C}$ & 4.23288 & -0.12907 & 0.04175 \\
$\mathrm{~N}$ & 5.38252 & -0.20109 & 0.07093 \\
& & &
\end{tabular}


22

Phenyl Transition State in Acetonitrile wb97xd/6-311+G(d,p)

$\begin{array}{llll}\mathrm{C} & 4.08206 & -0.19408 & -0.08753 \\ \mathrm{H} & 5.13493 & -0.42521 & -0.22067 \\ \mathrm{C} & 3.61587 & 1.09818 & 0.47058 \\ \mathrm{H} & 4.08889 & 1.90952 & -0.09942 \\ \mathrm{H} & 4.01475 & 1.19674 & 1.49158 \\ \mathrm{C} & 2.11186 & 1.11135 & 0.40806 \\ \mathrm{H} & 1.58894 & 1.99799 & 0.73991 \\ \mathrm{C} & 1.42000 & 0.02761 & -0.00205 \\ \mathrm{~N} & 1.93770 & -1.17039 & -0.43498 \\ \mathrm{O} & 3.33759 & -1.14215 & -0.44358 \\ \mathrm{C} & -2.85605 & 0.00548 & 0.00450 \\ \mathrm{C} & -2.16850 & 1.15153 & -0.40205 \\ \mathrm{H} & -2.71663 & 2.03143 & -0.71545 \\ \mathrm{C} & -0.78348 & 1.14974 & -0.41294 \\ \mathrm{H} & -0.25275 & 2.03240 & -0.74864 \\ \mathrm{C} & -0.06773 & 0.01889 & -0.01249 \\ \mathrm{C} & -0.76750 & -1.12455 & 0.38292 \\ \mathrm{H} & -0.21852 & -2.00577 & 0.68931 \\ \mathrm{C} & -2.15241 & -1.13627 & 0.39600 \\ \mathrm{H} & -2.68894 & -2.02289 & 0.71076 \\ \mathrm{C} & -4.28861 & -0.00020 & 0.01588 \\ \mathrm{~N} & -5.44119 & -0.00431 & 0.02583\end{array}$

25

Methoxyphenyl Minimum in Acetonitrile wb97xd/6-311+G(d,p)

$\begin{array}{lrrr}\mathrm{C} & 3.85339 & 0.06318 & -0.33346 \\ \mathrm{H} & 4.67918 & 0.10384 & -1.02983 \\ \mathrm{C} & 3.54995 & 1.16031 & 0.62695 \\ \mathrm{H} & 4.10592 & 2.07902 & 0.49341 \\ \mathrm{H} & 3.28589 & 0.90750 & 1.64699 \\ \mathrm{C} & 2.55678 & 0.81501 & -0.47457 \\ \mathrm{H} & 2.32946 & 1.51583 & -1.26610 \\ \mathrm{C} & 1.58857 & -0.23357 & -0.08386 \\ \mathrm{~N} & 2.14465 & -1.34415 & 0.25159 \\ \mathrm{O} & 3.52511 & -1.25056 & 0.06422 \\ \mathrm{C} & -2.64540 & 0.29994 & 0.00404 \\ \mathrm{O} & -3.96847 & 0.57365 & 0.03717 \\ \mathrm{C} & -4.88493 & -0.51103 & 0.05162 \\ \mathrm{H} & -5.87585 & -0.06356 & 0.08553 \\ \mathrm{H} & -4.73890 & -1.13902 & 0.93543 \\ \mathrm{H} & -4.79130 & -1.11902 & -0.85306 \\ \mathrm{C} & -2.10830 & -0.98946 & -0.02744 \\ \mathrm{H} & -2.74678 & -1.86257 & -0.03275 \\ \mathrm{C} & -0.73118 & -1.15847 & -0.06397 \\ \mathrm{H} & -0.32129 & -2.16085 & -0.10083 \\ \mathrm{C} & 0.13233 & -0.06265 & -0.06373 \\ \mathrm{C} & -0.42071 & 1.22304 & -0.03804 \\ \mathrm{H} & 0.22825 & 2.09180 & -0.03390 \\ \mathrm{C} & -1.79063 & 1.40556 & -0.00370 \\ \mathrm{H} & -2.21952 & 2.40021 & 0.01992\end{array}$


25

Methoxyphenyl Transition State in Acetonitrile wb97xd/6-311+G(d,p)

$\begin{array}{lccc}\text { C } & -4.23516 & -0.33369 & -0.07218 \\ \mathrm{H} & -5.27534 & -0.61079 & -0.21783 \\ \mathrm{C} & -3.83320 & 0.94908 & 0.55255 \\ \mathrm{H} & -4.22309 & 0.96738 & 1.58243 \\ \mathrm{H} & -4.36017 & 1.76304 & 0.03557 \\ \mathrm{C} & -2.33259 & 1.04689 & 0.47226 \\ \mathrm{H} & -1.85415 & 1.94707 & 0.83423 \\ \mathrm{C} & -1.58212 & 0.02445 & 0.00749 \\ \mathrm{~N} & -2.04319 & -1.17957 & -0.47409 \\ \mathrm{O} & -3.44419 & -1.22464 & -0.47356 \\ \mathrm{C} & 2.70751 & 0.25790 & -0.07218 \\ \mathrm{O} & 4.05191 & 0.43054 & -0.12152 \\ \mathrm{C} & 4.88248 & -0.66351 & 0.23175 \\ \mathrm{H} & 5.90596 & -0.31221 & 0.11781 \\ \mathrm{H} & 4.71573 & -1.51765 & -0.43190 \\ \mathrm{H} & 4.71684 & -0.96785 & 1.26990 \\ \mathrm{C} & 2.07790 & -0.92494 & 0.31109 \\ \mathrm{H} & 2.64973 & -1.79560 & 0.60372 \\ \mathrm{C} & 0.68708 & -0.99121 & 0.32481 \\ \mathrm{H} & 0.20714 & -1.91355 & 0.62985 \\ \mathrm{C} & -0.09695 & 0.10239 & -0.03307 \\ \mathrm{C} & 0.55207 & 1.27861 & -0.42434 \\ \mathrm{H} & -0.03356 & 2.13814 & -0.73117 \\ \mathrm{C} & 1.93293 & 1.36066 & -0.44195 \\ \mathrm{H} & 2.43170 & 2.27208 & -0.75068\end{array}$

21

Clorophenyl Minimum in Acetonitrile wb97xd/6-311+G(d,p)

$\begin{array}{lrrr}\mathrm{C} & -3.82213 & -0.20666 & -0.33026 \\ \mathrm{H} & -4.64632 & -0.29433 & -1.02392 \\ \mathrm{C} & -3.45169 & -1.28166 & 0.63074 \\ \mathrm{H} & -3.95343 & -2.23131 & 0.49965 \\ \mathrm{H} & -3.20009 & -1.01238 & 1.64966 \\ \mathrm{C} & -2.48354 & -0.88165 & -0.47442 \\ \mathrm{H} & -2.21798 & -1.56821 & -1.26646 \\ \mathrm{C} & -1.58069 & 0.22441 & -0.08927 \\ \mathrm{~N} & -2.19979 & 1.30097 & 0.24267 \\ \mathrm{O} & -3.56797 & 1.12587 & 0.06516 \\ \mathrm{C} & 2.65270 & -0.03291 & 0.00416 \\ \mathrm{C} & 2.05279 & 1.22106 & -0.01238 \\ \mathrm{H} & 2.65884 & 2.11809 & -0.00361 \\ \mathrm{C} & 0.67063 & 1.30561 & -0.05030 \\ \mathrm{H} & 0.19374 & 2.27764 & -0.07579 \\ \mathrm{C} & -0.11393 & 0.14824 & -0.06428 \\ \mathrm{C} & 0.51344 & -1.09798 & -0.05195 \\ \mathrm{H} & -0.07889 & -2.00562 & -0.05861 \\ \mathrm{C} & 1.89866 & -1.19608 & -0.01682 \\ \mathrm{H} & 2.38131 & -2.16491 & -0.00394 \\ \mathrm{Cl} & 4.39928 & -0.14394 & 0.04657\end{array}$


21

Chlorophenyl Transition State in Acetonitrile wb97xd/6-311+G(d,p)

$\mathrm{H} \quad 5.28121 \quad-0.41465 \quad-0.24515$

$\begin{array}{llll}\text { C } & 3.76328 & 1.09293 & 0.48254\end{array}$

$\begin{array}{llll}\mathrm{H} & 4.23576 & 1.91399 & -0.07399\end{array}$

$\mathrm{H} \quad 4.16453 \quad 1.17379 \quad 1.50454$

C $\quad 2.25883 \quad 1.10500 \quad 0.42257$

$\begin{array}{llll}\mathrm{H} & 1.73531 & 1.98848 & 0.76220\end{array}$

C $\quad 1.56468 \quad 0.02557 \quad 0.00405$

$\mathrm{N} \quad 2.08372 \quad-1.16726 \quad-0.44240$

$\begin{array}{llll}\mathrm{O} & 3.48429 & -1.13556 & -0.45946\end{array}$

C $\quad-2.70323 \quad 0.00596 \quad 0.00368$

C $\quad-2.02995 \quad 1.14438 \quad-0.41445$

$\begin{array}{llll}\mathrm{H} & -2.57906 & 2.01956 & -0.73852\end{array}$

$\begin{array}{llll}\text { C } & -0.64096 & 1.14004 & -0.42247\end{array}$

$\begin{array}{llll}\mathrm{H} & -0.11205 & 2.02129 & -0.76633\end{array}$

$\begin{array}{llll}\text { C } & 0.07645 & 0.01639 & -0.01017\end{array}$

$\begin{array}{llll}\text { C } & -0.62837 & -1.11826 & 0.39654\end{array}$

$\mathrm{H} \quad-0.08474 \quad-1.99901 \quad 0.71545$

$\begin{array}{llll}\text { C } & -2.01705 & -1.12997 & 0.40837\end{array}$

$\mathrm{H} \quad-2.55700 \quad-2.01095 \quad 0.73229$

$\begin{array}{llll}\mathrm{Cl} & -4.45761 & 0.00035 & 0.01465\end{array}$ 